\title{
Searching for heavily obscured post-AGB stars and planetary nebulae
}

\section{IRAS candidates with 2MASS PSC counterparts}

\author{
G. Ramos-Larios ${ }^{1, \star}$, M. A. Guerrero ${ }^{1}$, O. Suárez ${ }^{1,2}$, L. F. Miranda ${ }^{1}$, and J. F. Gómez ${ }^{1}$ \\ 1 Instituto de Astrofísica de Andalucía, CSIC. c/Camino Bajo de Huétor 50, 18008 Granada, Spain \\ e-mail: [gerardo; mar; lfm;jfg]@iaa.es \\ 2 UMR 6525 H. Fizeau, Université de Nice Sophia Antipolis, CNRS, OCA. Parc Valrose, 06108 Nice Cedex 2, France \\ e-mail: olga.suarez@unice.fr
}

Received 19 December 2008 / Accepted 24 February 2009

\begin{abstract}
Context. The transition from the asymptotic giant branch (AGB) to the planetary nebula (PN) phase is critical in the shaping of PNe. It has been suggested that the most asymmetric PNe are the descendants of massive AGB stars. Since these AGB stars are believed to have evolved into heavily obscured post-AGB stars and PNe, the compilation of a sample of bona fide obscured post-AGB stars and $\mathrm{PNe}$ is important for understanding the formation of asymmetric PNe.

Aims. We aim to identify and characterize a large number of post-AGB stars and PNe in the IR and to assess their degree of optical obscuration. The improved positions will enable future detailed studies to determine their true nature, whereas the optical and IR properties can be used to investigate their spectral behavior.

Methods. We used 2MASS, Spitzer GLIMPSE, MSX, and IRAS data in search of the near-IR counterparts of a sample of 165 presumably obscured IRAS post-AGB and PN candidates, and DSS red images to identify the optical counterparts among the objects detected in the near-IR. The IR spectral energy distributions (SEDs) in the wavelength range from $1 \mu \mathrm{m}$ to $100 \mu \mathrm{m}$ of the sources with unambiguous near-IR counterparts were analyzed using appropriate color-color diagrams.

Results. We have identified the near-IR counterparts of 119 sources out of the 165 IRAS post-AGB and PN candidates in our sample. The improved astrometric coordinates of these sources have allowed us to find optical counterparts for 59 of them, yielding a reduced sample of 60 optically obscured post-AGB star and PN candidates. Among the 119 sources with near-IR counterparts, only 80 have unambiguous identifications in the 2MASS Point Source Catalogue. For these sources, we find that objects with and without optical counterparts, while having similar mid- and far-IR colors, are segregated in color-color diagrams that use the near-IR $J$ band to compute one of the colors.
\end{abstract}

Key words. stars: AGB and post-AGB - stars: evolution - planetary nebulae: general - infrared: ISM

\section{Introduction}

Stars of low- and intermediate-mass $\left(0.8-1 M_{\odot}<M_{i}<\right.$ 8-10 $M_{\odot}$ ) experience heavy mass-loss episodes during the red giant phase that subsequently intensify at the tip of the asymptotic giant branch (AGB). With typical mass-loss rates $10^{-4}-10^{-5} M_{\odot} \mathrm{yr}^{-1}$, the AGB wind ejects most of the stellar envelope in a short time, and the star enters the post-AGB phase, from which it evolves into a planetary nebula (PN). The study of objects in the post-AGB phase, the short transition from the AGB to the PN phase, is essential to understanding the transformation of a spherically symmetric AGB envelope to an aspherical PN.

Objects in the transition from the AGB to the PN phase are strong far-infrared emitters, making them easily detectable by the Infrared Astronomical Satellite (IRAS). Furthermore, they are located in the [12]-[25] vs. [25]-[60] IRAS two-color diagram in a well-defined region (van der Veen \& Habing 1988). Therefore, the use of the IRAS two-color diagram provides an

^ Postdoctoral Research Fellow. invaluable strategy that has resulted in a number of catalogs of young PNe and their immediate precursors, post-AGB stars (e.g. Preite-Martinez 1988; Pottasch et al. 1988; van der Veen et al. 1989; Ratag et al. 1990; Hu et al. 1993; Pereira \& Miranda 2007). Recently, Suárez et al. (2006) presented a comprehensive optical spectroscopic atlas of post-AGB stars and young PNe selected from the IRAS Point Source Catalogue. In this work, a number of post-AGB candidates were so heavily obscured that no optical counterparts were found, while some others were not observed because they showed signs of strong obscuration in the optical.

Heavily obscured post-AGB stars and PNe represent objects whose circumstellar envelopes have not expanded enough to become optically thin. These objects may descend from the most massive AGB stars, as they are expected to be surrounded by very thick circumstellar envelopes during an important part or all of the post-AGB evolution as a result of the large amounts of mass ejected in their envelopes (up to $\sim 6 M_{\odot}$ for a star with initial mass $7 M_{\odot}$, Bloecker 1995). Moreover, the rapid evolution of the most massive post-AGB stars do not provide enough time 
for the circumstellar envelope to expand and become optically thin. Evidence of this evolutionary path is provided by young $\mathrm{PNe}$ whose very thick envelopes result in extremely high internal extinctions that prevent their detection in the optical range (e.g., IRAS 15103-5754, van de Steene \& Pottasch 1993; Suárez et al. 2006). Obscured post-AGB stars might be key objects for understanding the late evolution of the most massive PN progenitors.

With this work, we start a program devoted to searching heavily obscured post-AGB stars and PNe and to characterizing them in the IR. The observational material includes data available in the 2MASS database, the MSX catalog, and the Spitzer Space Telescope GLIMPSE Galactic plane survey, as well as recently acquired, more sensitive, higher resolution, near-IR observations. In this paper, the first in a series, we use 2MASS, MSX, and Spitzer GLIMPSE data to search for near-IR counterparts of presumably obscured IRAS post-AGB star and PN candidates. The accurate location of the sources with 2MASS counterparts has allowed us to determine whether these sources are really obscured in the optical or not. The 2MASS, MSX, and Spitzer GLIMPSE data have been used to characterize the near and midIR properties of the sources with 2MASS counterparts, allowing us to derive their spectral energy distributions (SED) in the IR domain. In a second paper, we present new, high-quality nearIR $J H K$ observations in order to search for the near-IR counterparts of the faintest objects, to find the near-IR counterparts of the objects not resolved by $2 \mathrm{MASS}$, to describe the morphology of sources with extended emission, and to investigate the variability of the objects with 2MASS counterparts.

The sample and archival data used in this paper are presented in Sects. 2 and 3, respectively. The identification of the IRAS sources with their 2MASS counterparts is described in Sect. 4, and their spectral properties are discussed in Sect. 5. Finally, a short summary is given in Sect. 6 .

\section{The sample}

The sample of IRAS post-AGB star and PN candidates investigated in this work is formed by the objects with no optical counterparts in the sample of Suárez et al. (2006), as well as by objects included in the original sample of these authors but not observed because they showed strong signs of heavy obscuration. These IRAS sources fulfill the criteria used by Suárez et al. (2006) to select objects with a dust temperature in their envelopes of $80-200 \mathrm{~K}$ and an expanding radius of $0.01-0.1 \mathrm{pc}$. These selection criteria are

(i) The sources are well-detected at 25 and $60 \mu \mathrm{m}$ in the IRAS Point Source Catalogue (see Beichman et al. 1988). The flux quality for each band must be

FQUAL $(12 \mu \mathrm{m}) \geq 1$,
FQUAL $(25 \mu \mathrm{m})=3$,
FQUAL $(60 \mu \mathrm{m})=3$.

(ii) The ratios between the IRAS photometric fluxes satisfy the following conditions:

$$
\begin{aligned}
& \frac{F_{v}(12 \mu \mathrm{m})}{F_{v}(25 \mu \mathrm{m})} \leq 0.50 ; \\
& \frac{F_{v}(25 \mu \mathrm{m})}{F_{v}(60 \mu \mathrm{m})} \geq 0.35 .
\end{aligned}
$$

(iii) For sources well-detected at $100 \mu \mathrm{m}$ in the IRAS Point Source Catalogue (FQUAL $(100 \mu \mathrm{m})=3)$, it is further imposed that

$$
\frac{F_{v}(60 \mu \mathrm{m})}{F_{v}(100 \mu \mathrm{m})} \geq 0.60 \text {. }
$$

(iv) The sources must show a low IRAS variability index:

$$
\mathrm{VAR} \leq 60 \% .
$$

The region of the IRAS two-color diagram defined by the previous selection criteria has a small overlap with objects of a different nature, mostly young stellar objects, $\mathrm{OH}$ stars, symbiotic stars, compact H II regions, and Seyfert galaxies. In particular, the IR SEDs, and thus the IR colors, of the circumstellar material around young stellar objects (e.g. Kenyon \& Hartmann 1995) are very similar to those of post-AGB stars and young PNe, making it difficult to discern the nature of nebulae around young and evolved objects. In contrast, Herbig Ae/Be stars, which exhibit distinct double-peak IR SEDs and are optically bright (e.g. Malfait et al. 1998; Meeus et al. 2001), are not easily mistaken for post-AGB stars and young PNe. To minimize the number of young stellar objects in the sample, two additional criteria have been imposed to our sample

(v) The sources are not classified in SIMBAD as young stars.

(vi) The sources are not located near the boundary of known star-forming regions.

With this criteria, the sample of IRAS post-AGB star and young PN candidates being presumably obscured sources consists of the 165 objects listed in Table 1. This table includes the IRAS names, coordinates, and error-ellipse (semi-major and semiminor axes and position angle of the major axis) of these objects, together with their fluxes and flux-quality factors in the IRAS $12 \mu \mathrm{m}, 25 \mu \mathrm{m}, 60 \mu \mathrm{m}$, and $100 \mu \mathrm{m}$ bands. The typical accuracy of the coordinates of the IRAS sources is $\sim 25^{\prime \prime}$, ranging from $\sim 10^{\prime \prime}$ up to $45^{\prime \prime}$ for the objects in Table 1 . The uncertainty in the IRAS coordinates is typically greater along the east-west direction. The location of these objects in the IRAS two-color diagram is illustrated in Fig. 1.

Some of the objects listed in Table 1 have been included in previous studies. High-quality images or detailed studies are available for IRAS 15452-5459 (Sahai et al. 2007), IRAS 17150-3224 (the Cotton Candy Nebula, Kwok et al. 1998; Hrivnak et al. 2006), IRAS 13356-6249, IRAS 135295934, IRAS 15144-5812, IRAS 17009-4154, and IRAS 170884221 (van de Steene et al. 2000), and IRAS 17516-2525 and IRAS 18135-1456 (Siódmiak et al. 2008). Near-IR photometric observations of IRAS 17404-2713, IRAS 17487-1922, IRAS 17516-2525, IRAS 18071-1727, and IRAS 19374+2359 (van der Veen et al. 1989), and spectroscopic observations of IRAS 17540-2753 and IRAS 18135-1456 (Oudmaijer et al. 1995) have also been reported.

\section{Archival data}

To identify and characterize the near- and mid-IR counterparts of the IRAS candidates to post-AGB stars and PNe in our sample, we searched the available 2MASS, MSX, and Spitzer GLIMPSE data products. The different properties of these catalogs are described below.

The 2 Microns All Sky Survey (2MASS) provides images of the complete sky in the $J(1.25 \mu \mathrm{m}), H(1.65 \mu \mathrm{m})$, and $K_{\mathrm{S}}(2.17 \mu \mathrm{m})$ bands (Skrutskie et al. 2006). The image pixel 
Table 1. IRAS identifications, positions and fluxes of post-AGB star and PN candidates.

\begin{tabular}{|c|c|c|c|c|c|c|c|c|c|c|}
\hline Name & $\begin{array}{c}\alpha \\
(\mathrm{J} 2000)\end{array}$ & $\begin{array}{c}\delta \\
(\mathrm{J} 2000)\end{array}$ & $\begin{array}{c}\text { Major Axis } \\
\left({ }^{\prime \prime}\right)\end{array}$ & $\begin{array}{c}\text { Minor Axis } \\
\left({ }^{\prime \prime}\right)\end{array}$ & $\begin{array}{l}\text { PA } \\
\left({ }^{\circ}\right)\end{array}$ & $\begin{array}{l}F_{12} \\
{[\mathrm{Jy}]}\end{array}$ & $\begin{array}{l}F_{25} \\
{[\mathrm{Jy}]}\end{array}$ & $\begin{array}{c}F_{60} \\
{[\mathrm{Jy}]}\end{array}$ & $\begin{array}{l}F_{100} \\
{[\mathrm{Jy}]}\end{array}$ & FQUAL \\
\hline IRAS 00509+6623 & 005407.7 & +664013 & 15 & 4 & 38 & 3.12 & 8.25 & 3.27 & 4.59 & 3331 \\
\hline IRAS 04137+7016 & 041908.8 & +702323 & 15 & 5 & 75 & 0.65 & 2.12 & 1.39 & 1.12 & 3331 \\
\hline IRAS $05573+3156$ & 060033.4 & +315643 & 15 & 5 & 89 & 7.95 & 47.93 & 80.21 & 108.90 & 3333 \\
\hline IRAS 06499+0145 & 065229.9 & +014200 & 17 & 5 & 96 & 0.93 & 15.16 & 27.60 & 46.16 & 3333 \\
\hline IRAS 08242-3828 & 082603.5 & -383848 & 29 & 6 & 116 & 11.58 & 26.77 & 22.53 & 10.75 & 3333 \\
\hline IRAS 08351-4634 & 083645.8 & -464446 & 14 & 4 & 117 & 15.02 & 31.02 & 12.78 & 8.81 & 3332 \\
\hline IRAS 09055-4629 & 090719.4 & -464121 & 15 & 4 & 126 & 0.74 & 3.05 & 1.47 & 32.70 & 3331 \\
\hline IRAS 09102-5101 & 091153.5 & -511351 & 12 & 4 & 129 & 0.56 & 3.73 & 4.87 & 35.11 & 2331 \\
\hline IRAS 09119-5150 & 091332.9 & -520239 & 14 & 3 & 129 & 0.79 & 3.82 & 2.13 & 25.94 & 3321 \\
\hline IRAS 09370-4826 & 093853.3 & -484010 & 24 & 5 & 124 & 10.82 & 30.14 & 14.16 & 5.41 & 3333 \\
\hline IRAS 09378-5117 & 093936.9 & -513126 & 15 & 3 & 136 & 1.87 & 6.43 & 3.01 & 16.31 & 3331 \\
\hline IRAS 09500-5236 & 095149.5 & -525040 & 14 & 3 & 147 & 0.58 & 2.23 & 4.49 & 18.44 & 3331 \\
\hline IRAS 10194-5625 & 102115.2 & -564032 & 17 & 5 & 146 & 16.75 & 57.02 & 27.78 & 52.91 & 3331 \\
\hline IRAS 11339-6004 & 113621.0 & -602056 & 18 & 3 & 143 & 1.96 & 8.45 & 4.24 & 23.02 & 3331 \\
\hline IRAS 11381-6401 & 114032.3 & -641833 & 14 & 3 & 146 & 5.41 & 27.76 & 17.02 & 72.42 & 3331 \\
\hline IRAS 11444-6150 & 114654.0 & -620709 & 14 & 3 & 157 & 3.22 & 14.33 & 13.42 & 76.35 & 2331 \\
\hline IRAS 11488-6432 & 115117.0 & -644909 & 15 & 5 & 144 & 1.56 & 3.37 & 3.07 & 14.49 & 3331 \\
\hline IRAS 11544-6408 & 115657.2 & -642511 & 15 & 4 & 142 & 12.49 & 29.81 & 12.52 & 12.57 & 3331 \\
\hline IRAS 11549-6225 & 115730.8 & -624212 & 12 & 3 & 149 & 7.60 & 20.06 & 10.79 & 33.30 & 3333 \\
\hline IRAS $12262-6417$ & 122904.2 & -643337 & 16 & 6 & 146 & 2.53 & 8.98 & 7.43 & 16.21 & 3331 \\
\hline IRAS $12309-5928$ & 123344.8 & -594518 & 15 & 4 & 139 & 1.99 & 5.44 & 2.93 & 19.26 & 3331 \\
\hline IRAS $12360-5740$ & 123853.2 & -575631 & 21 & 6 & 129 & 0.53 & 3.62 & 2.43 & 14.92 & 2331 \\
\hline IRAS 12405-6219 & 124331.1 & -623613 & 16 & 4 & 132 & 16.63 & 109.30 & 250.70 & 429.60 & 3332 \\
\hline IRAS $13293-6000$ & 133238.4 & -601536 & 16 & 5 & 129 & 0.75 & 1.95 & 4.99 & 34.31 & 3331 \\
\hline IRAS $13356-6249$ & 133905.8 & -630444 & 18 & 6 & 129 & 6.06 & 149.90 & 111.30 & 202.70 & 3331 \\
\hline IRAS 13398-5951 & 134312.2 & -600701 & 21 & 4 & 125 & 2.43 & 5.43 & 2.24 & 31.43 & 3331 \\
\hline IRAS 13404-6059 & 134352.8 & -611446 & 25 & 3 & 129 & 5.24 & 15.76 & 12.00 & 86.92 & 3331 \\
\hline IRAS $13421-6125$ & 134534.9 & -614007 & 20 & 5 & 128 & 15.21 & 31.17 & 13.93 & 122.10 & 3331 \\
\hline IRAS $13427-6531$ & 134625.7 & -654624 & 19 & 6 & 131 & 0.53 & 6.28 & 4.15 & 9.94 & 3331 \\
\hline IRAS 13483-5905 & 135144.1 & -592016 & 22 & 6 & 125 & 6.05 & 16.94 & 12.72 & 27.99 & 3331 \\
\hline IRAS 13500-6106 & 135334.5 & -612052 & 17 & 6 & 127 & 1.81 & 8.28 & 6.74 & 123.20 & 3331 \\
\hline IRAS 13529-5934 & 135624.8 & -594855 & 25 & 7 & 125 & 1.37 & 10.36 & 9.41 & 48.06 & 3331 \\
\hline IRAS 14104-5819 & 141400.4 & -583357 & 23 & 7 & 128 & 5.56 & 11.56 & 3.83 & 29.82 & 3331 \\
\hline IRAS $14249-5310$ & 142824.9 & -532406 & 21 & 5 & 117 & 11.82 & 29.00 & 23.20 & 9.63 & 3333 \\
\hline IRAS $14521-5300$ & 145545.9 & -531233 & 23 & 5 & 109 & 2.44 & 6.46 & 2.58 & 13.13 & 3331 \\
\hline IRAS $15038-5533$ & 150735.4 & -554454 & 22 & 5 & 110 & 1.22 & 3.40 & 1.61 & 84.62 & 3331 \\
\hline IRAS 15103-5754 & 151418.9 & -580520 & 18 & 3 & 2 & 10.80 & 101.50 & 126.50 & 103.40 & 3332 \\
\hline IRAS 15144-5812 & 151822.0 & -582313 & 17 & 4 & 116 & 7.67 & 35.59 & 35.89 & 149.50 & 3331 \\
\hline IRAS 15229-5433 & 152642.3 & -544423 & 17 & 5 & 110 & 0.42 & 3.84 & 2.27 & 80.68 & 2331 \\
\hline IRAS 15284-6026 & 153237.1 & -603704 & 23 & 5 & 109 & 2.10 & 9.97 & 10.88 & 32.46 & 3331 \\
\hline IRAS $15408-5657$ & 154448.1 & -570708 & 23 & 7 & 110 & 26.60 & 54.08 & 29.03 & 16.22 & 3322 \\
\hline IRAS $15408-5413$ & 154439.9 & -542305 & 29 & 4 & 107 & 166.90 & 350.60 & 111.00 & 154.20 & 3331 \\
\hline IRAS $15452-5459$ & 154911.5 & -550852 & 21 & 7 & 103 & 87.05 & 242.70 & 273.60 & 401.10 & 3331 \\
\hline IRAS 15531-5704 & 155710.4 & -571320 & 28 & 6 & 102 & 2.27 & 4.69 & 2.27 & 70.81 & 3331 \\
\hline IRAS $15534-5422$ & 155720.4 & -543040 & 25 & 6 & 106 & 1.06 & 8.27 & 12.41 & 355.80 & 3321 \\
\hline IRAS 16209-4714 & 162434.0 & -472129 & 21 & 4 & 96 & 1.17 & 18.87 & 20.68 & 63.87 & 3331 \\
\hline IRAS 16228-5014 & 162636.4 & -502105 & 13 & 5 & 146 & 0.79 & 25.68 & 66.78 & 159.60 & 3331 \\
\hline IRAS 16245-3859 & 162753.7 & -390546 & 43 & 9 & 99 & 3.78 & 25.27 & 31.62 & 16.13 & 3333 \\
\hline IRAS 16279-8158 & 163752.1 & -820449 & 11 & 4 & 102 & 4.21 & 10.31 & 5.70 & 2.35 & 3333 \\
\hline IRAS 16296-4507 & 163312.7 & -451344 & 17 & 3 & 97 & 3.26 & 17.67 & 14.20 & 31.32 & 3331 \\
\hline IRAS 16333-4807 & 163706.1 & -481342 & 17 & 4 & 97 & 9.33 & 42.98 & 89.26 & 113.20 & 3331 \\
\hline IRAS $16507-4810$ & 165431.0 & -481521 & 33 & 11 & 99 & 1.08 & 12.56 & 8.96 & 13.48 & 3222 \\
\hline IRAS 16517-3626 & 165505.1 & -363130 & 34 & 5 & 100 & 1.51 & 5.97 & 2.27 & 11.38 & 3331 \\
\hline IRAS $16518-3425$ & 165508.4 & -343010 & 24 & 6 & 120 & 1.02 & 7.98 & 1.66 & 5.16 & 3331 \\
\hline IRAS 16558-3417 & 165910.5 & -342205 & 30 & 6 & 98 & 2.63 & 11.60 & 7.68 & 10.85 & 3332 \\
\hline IRAS 16559-2957 & 165908.1 & -300140 & 19 & 5 & 98 & 9.17 & 32.37 & 16.38 & 4.19 & 3331 \\
\hline IRAS 16567-3838 & 170008.2 & -384308 & 40 & 5 & 99 & 7.30 & 15.68 & 7.07 & 58.57 & 3331 \\
\hline IRAS $16584-3710$ & 170152.5 & -371457 & 38 & 6 & 99 & 0.91 & 6.14 & 4.39 & 25.12 & 3331 \\
\hline IRAS 17009-4154 & 170429.7 & -415835 & 33 & 6 & 99 & 7.44 & 86.38 & 65.84 & 260.00 & 3331 \\
\hline IRAS $17010-3810$ & 170426.9 & -381443 & 24 & 6 & 99 & 2.23 & 14.78 & 7.68 & 30.04 & 3331 \\
\hline IRAS 17021-3109 & 170523.2 & -311318 & 37 & 5 & 97 & 3.29 & 11.97 & 16.59 & 7.88 & 3333 \\
\hline IRAS 17021-3054 & 170524.1 & -305813 & 23 & 5 & 97 & 1.18 & 5.34 & 2.49 & 16.78 & 3331 \\
\hline IRAS 17052-3245 & 170832.7 & -324943 & 35 & 6 & 97 & 3.13 & 10.79 & 12.02 & 10.12 & 3331 \\
\hline
\end{tabular}


Table 1. continued.

\begin{tabular}{|c|c|c|c|c|c|c|c|c|c|c|}
\hline Name & $\begin{array}{c}\alpha \\
(\mathrm{J} 2000)\end{array}$ & $\begin{array}{c}\delta \\
(\mathrm{J} 2000)\end{array}$ & $\begin{array}{c}\text { Major Axis } \\
\left({ }^{\prime \prime}\right)\end{array}$ & $\begin{array}{c}\text { Minor Axis } \\
\left({ }^{\prime \prime}\right)\end{array}$ & $\begin{array}{l}\text { PA } \\
\left({ }^{\circ}\right)\end{array}$ & $\begin{array}{c}F_{12} \\
{[\mathrm{Jy}]}\end{array}$ & $\begin{array}{c}F_{25} \\
{[\mathrm{Jy}]}\end{array}$ & $\begin{array}{c}F_{60} \\
{[\mathrm{Jy}]}\end{array}$ & $\begin{array}{l}F_{100} \\
{[\mathrm{Jy}]}\end{array}$ & FQUAL $^{\dagger}$ \\
\hline IRAS 17067-3759 & 171008.3 & -380322 & 29 & 6 & 98 & 1.98 & 10.60 & 10.66 & 68.33 & 3331 \\
\hline IRAS $17088-4221$ & 171222.6 & -422513 & 26 & 5 & 98 & 42.70 & 128.30 & 106.80 & 36.90 & 3333 \\
\hline IRAS 17097-3624 & 171305.1 & -362754 & 42 & 6 & 97 & 1.52 & 6.62 & 10.11 & 227.40 & 3331 \\
\hline IRAS $17149-3053$ & 171811.7 & -305640 & 36 & 6 & 96 & 2.13 & 11.12 & 7.82 & 20.93 & 3331 \\
\hline IRAS $17150-3224$ & 171819.9 & -322720 & 32 & 6 & 96 & 57.92 & 322.30 & 268.30 & 82.41 & 3333 \\
\hline IRAS $17153-3814$ & 171844.6 & -381723 & 15 & 4 & 97 & 2.64 & 19.55 & 52.88 & 436.50 & 3331 \\
\hline IRAS 17158-4049 & 171920.9 & -405243 & 46 & 6 & 97 & 0.99 & 6.56 & 7.14 & 295.40 & 3331 \\
\hline IRAS 17168-3736 & 172015.0 & -373932 & 18 & 5 & 97 & 9.79 & 36.98 & 47.89 & 280.60 & 3331 \\
\hline IRAS 17175-2819 & 172042.4 & -282236 & 42 & 5 & 95 & 3.38 & 14.94 & 5.23 & 10.18 & 3331 \\
\hline IRAS $17233-2602$ & 172628.7 & -260458 & 27 & 5 & 94 & 3.69 & 10.61 & 7.19 & 7.93 & 3331 \\
\hline IRAS 17234-4008 & 172655.6 & -401103 & 37 & 6 & 96 & 1.48 & 12.46 & 10.48 & 83.69 & 3331 \\
\hline IRAS 17269-2235 & 172958.2 & -223734 & 26 & 7 & 93 & 0.68 & 14.89 & 22.26 & 8.85 & 2333 \\
\hline IRAS $17291-2147$ & 173210.1 & -214959 & 24 & 6 & 93 & 2.54 & 12.18 & 8.24 & 6.42 & 3331 \\
\hline IRAS $17301-2538$ & 173313.2 & -254024 & 20 & 5 & 93 & 0.78 & 7.80 & 4.29 & 33.13 & 3331 \\
\hline IRAS 17348-2906 & 173804.2 & -290823 & 39 & 9 & 93 & 4.71 & 11.55 & 8.24 & 305.50 & 3331 \\
\hline IRAS 17359-2902 & 173907.7 & -290402 & 41 & 9 & 93 & 2.04 & 12.38 & 7.61 & 259.60 & 3321 \\
\hline IRAS $17360-2142$ & 173905.9 & -214352 & 25 & 7 & 92 & 0.93 & 12.53 & 12.46 & 6.52 & 3331 \\
\hline IRAS $17361-4159$ & 173943.8 & -420041 & 25 & 5 & 95 & 2.58 & 6.73 & 5.54 & 24.89 & 3331 \\
\hline IRAS $17376-3448$ & 174056.4 & -345003 & 22 & 5 & 93 & 3.70 & 10.18 & 6.92 & 13.33 & 3331 \\
\hline IRAS $17382-2531$ & 174120.1 & -253253 & 38 & 5 & 92 & 3.03 & 7.64 & 5.31 & 11.46 & 3331 \\
\hline IRAS $17385-3332$ & 174152.2 & -333341 & 22 & 6 & 93 & 2.88 & 13.25 & 10.01 & 238.40 & 3331 \\
\hline IRAS $17385-2413$ & 174138.3 & -241441 & 32 & 6 & 92 & 3.73 & 7.67 & 3.70 & 9.93 & 3331 \\
\hline IRAS 17393-2727 & 174232.2 & -272828 & 40 & 7 & 92 & 1.83 & 17.83 & 36.85 & 85.69 & 2331 \\
\hline IRAS 17404-2713 & 174339.4 & -271418 & 20 & 6 & 92 & 3.99 & 20.74 & 15.49 & 83.59 & 3331 \\
\hline IRAS 17418-3335 & 174510.7 & -333613 & 18 & 5 & 94 & 1.83 & 14.63 & 12.06 & 161.90 & 3331 \\
\hline IRAS $17443-2949$ & 174735.2 & -295056 & 34 & 6 & 93 & 15.77 & 39.39 & 34.47 & 354.10 & 3331 \\
\hline IRAS $17479-3032$ & 175112.5 & -303344 & 33 & 7 & 92 & 2.63 & 13.02 & 16.51 & 106.10 & 2321 \\
\hline IRAS 17482-2501 & 175122.3 & -250150 & 27 & 8 & 91 & 1.34 & 5.18 & 11.53 & 381.60 & 2331 \\
\hline IRAS $17487-1922$ & 175144.7 & -192342 & 31 & 8 & 91 & 2.16 & 20.25 & 7.21 & 11.81 & 3331 \\
\hline IRAS $17499-3520$ & 175319.7 & -352111 & 30 & 6 & 92 & 2.82 & 13.10 & 4.02 & 6.10 & 3331 \\
\hline IRAS 17506-2955 & 175349.3 & -295534 & 21 & 6 & 91 & 1.62 & 6.29 & 6.61 & 194.40 & 3331 \\
\hline IRAS $17516-2525$ & 175443.5 & -252627 & 29 & 6 & 91 & 51.58 & 115.60 & 100.10 & 292.10 & 3331 \\
\hline IRAS $17540-2753$ & 175714.1 & -275416 & 16 & 4 & 91 & 1.39 & 14.19 & 26.16 & 341.00 & 2331 \\
\hline IRAS $17543-3102$ & 175734.2 & -310300 & 27 & 6 & 91 & 2.76 & 21.87 & 24.44 & 71.27 & 3331 \\
\hline IRAS $17548-2753$ & 175757.7 & -275320 & 29 & 5 & 91 & 1.27 & 16.95 & 21.95 & 174.90 & 3331 \\
\hline IRAS $17550-2800$ & 175810.6 & -280026 & 37 & 5 & 91 & 2.52 & 5.89 & 5.96 & 203.40 & 3331 \\
\hline IRAS $17550-2120$ & 175804.9 & -212107 & 32 & 5 & 90 & 5.38 & 21.08 & 30.81 & 43.64 & 3331 \\
\hline IRAS $17552-2030$ & 175816.3 & -203022 & 37 & 6 & 90 & 1.22 & 4.62 & 3.93 & 29.08 & 3331 \\
\hline IRAS 17560-2027 & 175904.4 & -202723 & 24 & 6 & 90 & 1.59 & 15.32 & 17.79 & 40.99 & 3331 \\
\hline IRAS $17580-3111$ & 180119.7 & -311123 & 38 & 7 & 91 & 3.24 & 15.32 & 7.96 & 15.19 & 3331 \\
\hline IRAS 17582-2619 & 180121.2 & -261937 & 24 & 8 & 90 & 1.38 & 9.28 & 7.92 & 293.30 & 3331 \\
\hline IRAS 17596-3952 & 180306.2 & -395155 & 34 & 10 & 92 & 0.48 & 1.11 & 0.59 & 12.68 & 3221 \\
\hline IRAS $18011-1847$ & 180402.7 & -184710 & 32 & 5 & 89 & 2.54 & 13.67 & 16.30 & 60.24 & 3331 \\
\hline IRAS $18015-1352$ & 180422.2 & -135149 & 29 & 7 & 89 & 2.11 & 4.24 & 3.37 & 21.74 & 3332 \\
\hline IRAS 18016-2743 & 180445.8 & -274311 & 24 & 5 & 90 & 3.02 & 7.15 & 3.07 & 131.80 & 3331 \\
\hline IRAS $18039-1903$ & 180653.3 & -190309 & 37 & 6 & 89 & 4.17 & 11.11 & 7.31 & 261.90 & 3321 \\
\hline IRAS $18049-2118$ & 180754.8 & -211809 & 42 & 6 & 89 & 11.83 & 25.70 & 9.12 & 206.50 & 3331 \\
\hline IRAS 18051-2415 & 180812.8 & -241436 & 38 & 6 & 90 & 1.88 & 8.14 & 9.49 & 172.30 & 3331 \\
\hline IRAS $18071-1727$ & 181005.4 & -172656 & 26 & 7 & 89 & 23.66 & 76.57 & 83.46 & 304.70 & 3331 \\
\hline IRAS 18083-2155 & 181118.9 & -215505 & 24 & 6 & 89 & 7.40 & 37.97 & 27.07 & 496.00 & 3331 \\
\hline IRAS $18087-1440$ & 181134.2 & -143959 & 28 & 7 & 88 & 2.58 & 21.86 & 32.31 & 217.20 & 3331 \\
\hline IRAS 18105-1935 & 181332.2 & -193503 & 22 & 7 & 89 & 7.33 & 19.87 & 10.46 & 369.50 & 3331 \\
\hline IRAS 18113-2503 & 181426.2 & -250255 & 33 & 6 & 89 & 2.90 & 14.77 & 12.90 & 29.16 & 3331 \\
\hline IRAS $18135-1456$ & 181625.6 & -145515 & 20 & 6 & 88 & 31.02 & 124.40 & 157.60 & 429.10 & 3331 \\
\hline IRAS $18183-2538$ & 182124.7 & -253635 & 22 & 6 & 88 & 1.64 & 3.91 & 2.05 & 33.40 & 3331 \\
\hline IRAS 18199-1442 & 182250.8 & -144049 & 22 & 6 & 87 & 12.05 & 25.54 & 22.25 & 282.60 & 3321 \\
\hline IRAS $18229-1127$ & 182545.0 & -112556 & 15 & 6 & 87 & 6.28 & 27.86 & 37.09 & 133.50 & 3232 \\
\hline IRAS $18236-0447$ & 182620.3 & -044542 & 41 & 6 & 86 & 2.08 & 5.17 & 5.06 & 12.12 & 3331 \\
\hline IRAS 18246-1032 & 182724.0 & -103024 & 30 & 6 & 86 & 2.19 & 20.25 & 50.37 & 386.00 & 3331 \\
\hline IRAS $18355-0712$ & 183815.4 & -070952 & 31 & 5 & 85 & 1.73 & 14.34 & 31.14 & 159.90 & 2321 \\
\hline IRAS $18361-1203$ & 183858.8 & -120044 & 37 & 6 & 86 & 2.24 & 4.83 & 3.33 & 52.79 & 3331 \\
\hline IRAS $18385+1350$ & 184052.0 & +135253 & 13 & 4 & 26 & 0.49 & 3.34 & 3.61 & 3.83 & 3333 \\
\hline IRAS 18434-0042 & 184604.4 & -003855 & 30 & 6 & 83 & 1.97 & 5.61 & 3.25 & 343.40 & 3321 \\
\hline IRAS $18454+0001$ & 184801.5 & +000447 & 39 & 8 & 83 & 0.80 & 14.54 & 13.61 & 383.50 & 2331 \\
\hline
\end{tabular}


Table 1. continued.

\begin{tabular}{|c|c|c|c|c|c|c|c|c|c|c|}
\hline Name & $\begin{array}{c}\alpha \\
(\mathrm{J} 2000)\end{array}$ & $\begin{array}{c}\delta \\
(\mathrm{J} 2000)\end{array}$ & $\begin{array}{c}\text { Major Axis } \\
\left({ }^{\prime \prime}\right)\end{array}$ & $\begin{array}{c}\text { Minor Axis } \\
\left({ }^{\prime \prime}\right)\end{array}$ & $\begin{array}{l}\text { PA } \\
\left({ }^{\circ}\right)\end{array}$ & $\begin{array}{r}F_{12} \\
{[\mathrm{Jy}]}\end{array}$ & $\begin{array}{r}F_{25} \\
{[\mathrm{Jy}]}\end{array}$ & $\begin{array}{r}F_{60} \\
{[\mathrm{Jy}]}\end{array}$ & $\begin{array}{l}F_{100} \\
{[\mathrm{Jy}]}\end{array}$ & $\mathrm{FQUAL}^{\dagger}$ \\
\hline IRAS $18470+0015$ & 184939.1 & +001852 & 38 & 6 & 83 & 4.11 & 10.67 & 8.07 & 249.60 & 3331 \\
\hline IRAS $18485+0642$ & 185058.9 & +064555 & 18 & 6 & 80 & 3.58 & 21.86 & 25.32 & 61.08 & 3331 \\
\hline IRAS 18514+0019 & 185357.9 & +002324 & 29 & 6 & 81 & 4.95 & 23.38 & 17.26 & 152.20 & 3331 \\
\hline IRAS $18524+0544$ & 185454.1 & +054811 & 21 & 5 & 75 & 0.37 & 5.61 & 5.04 & 39.88 & 3331 \\
\hline IRAS $18529+0210$ & 185526.3 & +021449 & 22 & 6 & 81 & 5.69 & 16.24 & 15.24 & 1921.00 & 3331 \\
\hline IRAS $18576+0341$ & 190010.9 & +034547 & 23 & 5 & 81 & 58.48 & 425.00 & 274.70 & 1660.00 & 3331 \\
\hline IRAS $18580+0818$ & 190025.2 & +082246 & 27 & 6 & 79 & 0.90 & 3.35 & 2.31 & 16.39 & 3331 \\
\hline IRAS $18596+0315$ & 190206.2 & +032016 & 24 & 8 & 80 & 2.60 & 14.17 & 22.57 & 112.80 & 3331 \\
\hline IRAS $19006+1022$ & 190259.9 & +102635 & 29 & 8 & 79 & 1.02 & 5.34 & 6.58 & 12.85 & 3331 \\
\hline IRAS $19011+1049$ & 190330.7 & +105353 & 50 & 6 & 79 & 9.40 & 26.10 & 12.33 & 9.26 & 3331 \\
\hline IRAS $19013+0629$ & 190344.4 & +063412 & 30 & 12 & 79 & 5.12 & 17.60 & 16.31 & 79.21 & 3331 \\
\hline IRAS $19015+1256$ & 190352.6 & +130121 & 23 & 5 & 38 & 0.92 & 4.20 & 1.33 & 7.60 & 3321 \\
\hline IRAS $19071+0857$ & 190929.7 & +090223 & 26 & 9 & 79 & 0.94 & 17.26 & 13.06 & 72.03 & 3331 \\
\hline IRAS $19075+0432$ & 191000.0 & +043706 & 27 & 8 & 80 & 5.25 & 28.13 & 31.77 & 14.36 & 3333 \\
\hline IRAS 19079-0315 & 191032.4 & -031016 & 23 & 7 & 81 & 2.27 & 12.03 & 4.22 & 16.56 & 3331 \\
\hline IRAS $19083+0119$ & 191054.4 & +012442 & 21 & 7 & 80 & 2.34 & 15.58 & 14.27 & 4.72 & 3332 \\
\hline IRAS 19094+1627 & 191144.5 & +163254 & 25 & 7 & 78 & 0.80 & 5.17 & 2.68 & 5.85 & 3331 \\
\hline IRAS 19134+2131 & 191535.4 & +213633 & 32 & 5 & 77 & 5.06 & 15.56 & 8.56 & 3.95 & 3331 \\
\hline IRAS $19176+1251$ & 191955.8 & +125734 & 27 & 6 & 78 & 0.85 & 10.70 & 4.11 & 71.30 & 3331 \\
\hline IRAS $19178+1206$ & 192014.0 & +121220 & 17 & 6 & 78 & 3.66 & 8.57 & 4.85 & 68.21 & 3331 \\
\hline IRAS 19181+1806 & 192025.2 & +181141 & 37 & 5 & 78 & 0.47 & 4.14 & 7.38 & 14.09 & 2331 \\
\hline IRAS $19190+1102$ & 192125.3 & +110840 & 28 & 5 & 78 & 1.59 & 13.67 & 24.52 & 20.41 & 3333 \\
\hline IRAS 19193+1804 & 192131.5 & +181009 & 27 & 6 & 78 & 0.54 & 9.11 & 15.84 & 58.62 & 3331 \\
\hline IRAS $19208+1541$ & 192306.8 & +154733 & 26 & 5 & 78 & 0.77 & 1.57 & 3.37 & 92.78 & 3331 \\
\hline IRAS $19315+2235$ & 193341.6 & +224208 & 16 & 5 & 74 & 1.05 & 5.68 & 4.41 & 8.80 & 3331 \\
\hline IRAS 19319+2214 & 193403.5 & +222116 & 24 & 4 & 74 & 3.18 & 6.58 & 3.16 & 39.29 & 3331 \\
\hline IRAS $19374+2359$ & 193935.6 & +240628 & 32 & 5 & 71 & 23.62 & 98.18 & 70.87 & 768.40 & 3331 \\
\hline IRAS 19454+2920 & 194724.2 & +292811 & 19 & 5 & 69 & 17.27 & 89.56 & 54.43 & 14.66 & 3333 \\
\hline IRAS $20035+3242$ & 200529.6 & +325135 & 20 & 3 & 66 & 7.49 & 25.59 & 21.16 & 16.39 & 3332 \\
\hline IRAS $20042+3259$ & 200610.6 & +330751 & 22 & 5 & 64 & 1.49 & 4.40 & 1.89 & 41.49 & 3331 \\
\hline IRAS 20174+3222 & 201928.0 & +323213 & 21 & 5 & 64 & 2.44 & 16.02 & 8.04 & 8.73 & 3332 \\
\hline IRAS 20214+3749 & 202319.2 & +375852 & 22 & 5 & 67 & 1.95 & 4.21 & 2.82 & 184.10 & 3321 \\
\hline IRAS 20244+3509 & 202625.4 & +351914 & 20 & 6 & 67 & 1.27 & 4.31 & 4.36 & 179.30 & 3331 \\
\hline IRAS 20406+2953 & 204245.9 & +300406 & 36 & 5 & 67 & 12.48 & 68.37 & 49.77 & 13.00 & 3332 \\
\hline IRAS $20461+3853$ & 204804.6 & +390500 & 31 & 5 & 57 & 2.10 & 11.28 & 4.80 & 4.46 & 3331 \\
\hline IRAS $21525+5643$ & 215415.0 & +565723 & 9 & 3 & 15 & 4.33 & 11.62 & 8.93 & 20.47 & 3331 \\
\hline IRAS $21537+6435$ & 215504.5 & +644954 & 12 & 4 & 161 & 6.91 & 26.10 & 13.34 & 6.07 & 3331 \\
\hline IRAS 21554+6204 & 215658.1 & +621843 & 8 & 4 & 9 & 62.94 & 138.20 & 52.13 & 15.59 & 3333 \\
\hline
\end{tabular}

$\dagger$ The IRAS flux-quality factor, FQUAL, indicates whether the flux values are of high quality (3), moderate quality (2), or simply represent an upper limit of the flux (1). The four values in this column refer consecutively to the flux quality in the $12 \mu \mathrm{m}, 25 \mu \mathrm{m}, 60 \mu \mathrm{m}$, and $100 \mu \mathrm{m}$ bands.

scale is $1^{\prime \prime}$ and the typical spatial resolution in the survey was $\sim 5^{\prime \prime}$, as derived from the $F W H M$ of the stars in the images. The $10 \sigma$ detection limit for point sources is $J<15.8 \mathrm{mag}$, $H<15.1 \mathrm{mag}$, and $K_{\mathrm{S}}<14.3 \mathrm{mag}$.

The Mid-course Space Experiment $(M S X)$ provides a detailed mid-IR map of a narrow strip, $|b| \leq 5^{\circ}$, of the whole Galactic plane in four different bands, namely bands $A(8.28 \mu \mathrm{m}), C(12.13 \mu \mathrm{m}), D(14.65 \mu \mathrm{m})$, and $E(21.3 \mu \mathrm{m})$. The positional uncertainty in the $M S X$ Point Source Catalogue (MSX6C) is of the order of 2", while the spatial resolution is quoted as $18^{\prime \prime} .3$ (Price et al. 2001). The sensitivity levels for the bands $A, C, D$, and $E$ are $160 \mathrm{mJy}, 1200 \mathrm{mJy}, 1000 \mathrm{mJy}$, and $3000 \mathrm{mJy}$, respectively.

The Galactic Legacy Infrared Midplane Survey Extraordinaire (GLIMPSE) project obtained Spitzer Infrared Array Camera (IRAC) observations of the Galactic plane within the latitude and longitude ranges $|b| \leq 1^{\circ}$, and $|l|=10^{\circ}-65^{\circ}$, respectively, in four bands with wavelengths (and bandwidths) $3.550 \mu \mathrm{m}(0.75 \mu \mathrm{m}), 4.493 \mu \mathrm{m}(1.9015 \mu \mathrm{m}), 5.731 \mu \mathrm{m}$
$(1.425 \mu \mathrm{m})$, and $7.872 \mu \mathrm{m}(2.905 \mu \mathrm{m})$. These results were subsequently processed to yield the GLIMPSE Image Atlas with a pointing accuracy of $\sim 0^{\prime} \cdot 3$, and a spatial resolution that varies between 1'.7 and 2'.0 (Fazio et al. 2004). The sensitivity (saturation levels) for the the different bands are $0.5 \mathrm{mJy}$ (439 mJy) in the $3.6 \mu \mathrm{m}$ band, $0.5 \mathrm{mJy}(450 \mathrm{mJy})$ in the $4.5 \mu \mathrm{m}$ band, $2 \mathrm{mJy}(2930 \mathrm{mJy})$ in the $5.8 \mu \mathrm{m}$ band, and $5 \mathrm{mJy}$ (1590 mJy) in the $8.0 \mu \mathrm{m}$ band.

\section{Source identification}

The accuracy of the coordinates of an IRAS source may not be good enough to allow a straightforward identification of its counterpart in the 2MASS, MSX or GLIMPSE catalogs. This is especially the case of IRAS sources in crowded fields located along the Galactic plane or near the Galactic center, for which the error-ellipse typically includes several near-IR sources. For defining a strategy to search for the near- and mid-IR 


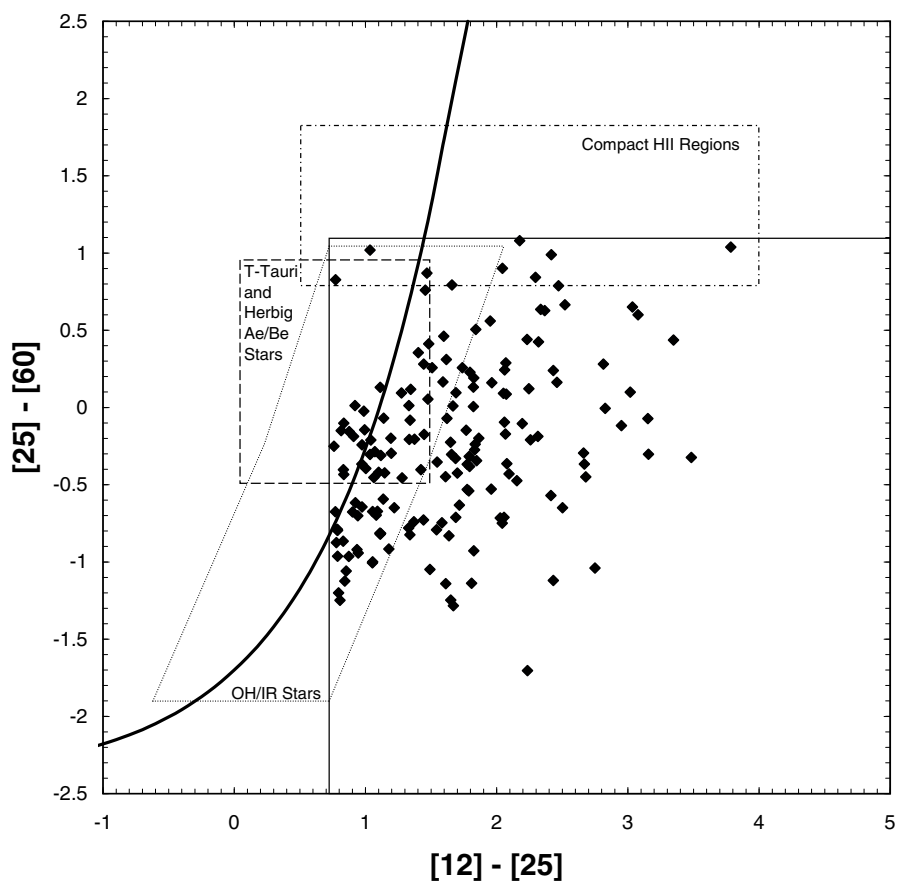

Fig. 1. IRAS color-color diagram showing the location of the IRAS sources in our sample. The thin solid lines represent our selection criteria, while the solid thick line represents the expected colors for AGB stars (Bedijn 1987). The loci of OH/IR stars (Sivagnaman 1989; te Lintel Hekkert et al. 1991), T-Tauri and Herbig Ae/Be stars (Harris et al. 1988), and compact H II regions (Antonopoulos \& Pottasch 1987) that overlap with post-AGB stars and $\mathrm{PNe}$ are also overlaid on the diagram. IRAS colors are computed as $[A]-[B]=2.5 \log \left(F_{v}(B) / F_{v}(A)\right)$.

counterparts of the IRAS sources in Table 1, a comparison between the three databases used in this work can be useful.

The 2MASS images provide a complete coverage of the sky with a spatial resolution $\left(\sim 5^{\prime \prime}\right)$ better than the positional uncertainty of the IRAS sources in Table 1 . However, the sensitivity of the 2MASS images to obscured sources is limited because of the strong extinction and intrinsic red colors of these sources. Accordingly, identifying the 2MASS counterparts of the IRAS sources in our sample is not obvious in general. This is not the case for the GLIMPSE Image Atlas that is highly sensitive to mid-IR sources and that provides better spatial resolution $\left(\leq 2{ }^{\prime \prime} 0\right)$ than the MSX and 2MASS images. This database thus seems especially suited to accurately determining the position of the IRAS sources in Table 1. Unfortunately, its limited spatial coverage results in a reduced number of sources with available GLIMPSE data. On the other hand, the MSX data have extremely high sensitivity to obscured sources and its coverage is good enough to include a significant fraction of the sources in our sample. The $M S X$ images, however, have poor spatial resolution $\left(\sim 18^{\prime \prime}\right)$ that can make it difficult to pinpoint the near-IR counterpart.

Given the different properties of the 2MASS, MSX, and GLIMPSE Image Atlas, we proceeded as follows in order to identify the near-IR counterparts of the IRAS sources. When available, we first examined the GLIMPSE images to pinpoint the accurate location of the IRAS sources, thus allowing us to find its near-IR counterpart in 2MASS images. This procedure is illustrated in Fig. 2 for IRAS 17359-2902, showing that the bright Spitzer mid-IR source inside its IRAS error-ellipse allows the unambiguous identification of its near-IR counterpart among the near-IR sources within this ellipse in the 2MASS image. The use of the GLIMPSE images resulted in identification of the near-IR counterparts of 22 sources in Table 1, although it must be noticed that some heavily obscured Spitzer sources were not detected in the 2MASS images We then examined the MSX data of the sources with no GLIMPSE data available and were able to find the near-IR counterparts for 52 additional sources in Table 1. A typical example of identifying the near-IR counterpart of an IRAS source using $M S X$ data is shown in Fig. 2 for IRAS 17010-3810. The position of the MSX source MSX6C G347.4963+01.8505, coincident within $1^{\prime \prime}$ with the position of the source 2MASS 17042731-3814417 (marked in the 2MASS image), has allowed us to determine that this is the near-IR counterpart of IRAS 17010-3810. Finally, we examined the JHK 2MASS images of the IRAS sources with no GLIMPSE and $M S X$ data available, and selected the near-IR counterparts that appear as isolated sources within the error-ellipse of the IRAS source and whose near-IR colors are typical of post-AGB stars or PNe, i.e., $(J-H) \sim 1.0$ and $(H-K) \sim 0.4$ (García-Lario et al. 1997). This has added an additional 43 IRAS sources with 2MASS counterparts. The result is a total of 119 sources with near-IR emission out of the sample of 165 sources listed in Table 1.

Among these 119 IRAS sources detected in the near-IR, there are 80 sources with a reliable counterpart in the 2MASS Point Source Catalogue (PSC). The remaning 39 IRAS sources in Table 1 have near-IR emission detected in 2MASS, but assigning a counterpart in the 2MASS PSC is dubious due either to their extended appearance in the 2MASS images, to the possible contamination by an adjacent source, or to their extreme faintness, at the detection limit of 2MASS.

The sample of the 80 IRAS post-AGB and PN candidates in Table 1 with a counterpart in the 2MASS PSC is listed in Table 2 with their coordinates and near-IR magnitudes, and their identification charts are shown in Fig. 3. The angular separation with the position of the IRAS source is also listed in this table. The distance between the IRAS and 2MASS coordinates is typically below $5^{\prime \prime}$, i.e., within the ellipse-error of the IRAS coordinates, with no preferential direction of the distance vector. In a few cases, the offset between the IRAS and 2MASS coordinates is greater than the IRAS coordinates uncertainty. Obviously, these are sources that have been identified through their GLIMPSE Spitzer or MSX images. A close inspection of the original IRAS images reveals that these sources are either extended sources or multiple, unresolved sources, thus introducing a significant offset in their IRAS coordinates.

The sample of the 39 IRAS post-AGB and PN candidates in Table 1 with a 2MASS counterpart but dubious assignation in the 2MASS PSC are listed in Table 3 and their identification charts are shown in Fig. 4 (which is at the end of the text).

Finally, the 46 sources that do not have 2MASS counterparts are listed in Table 4. Most of these sources have been observed by MSX and many of them even have Spitzer GLIMPSE observations, but no near-IR counterpart is detected in 2MASS at the improved astrometric positions provided by the MSX and Spitzer GLIMPSE catalogs. Only IRAS 16245-3859, IRAS 18385+1350, and IRAS 21537+6435 have neither Spitzer GLIMPSE nor MSX observations and no obvious near-IR counterpart in 2MASS images within their IRAS ellipse-error. The study of sources in Tables 3 and 4 requires near-IR observations of greater spatial resolution and sensitivity, respectively, than the 2MASS observations used in this paper. These observations will be presented in a subsequent paper. 

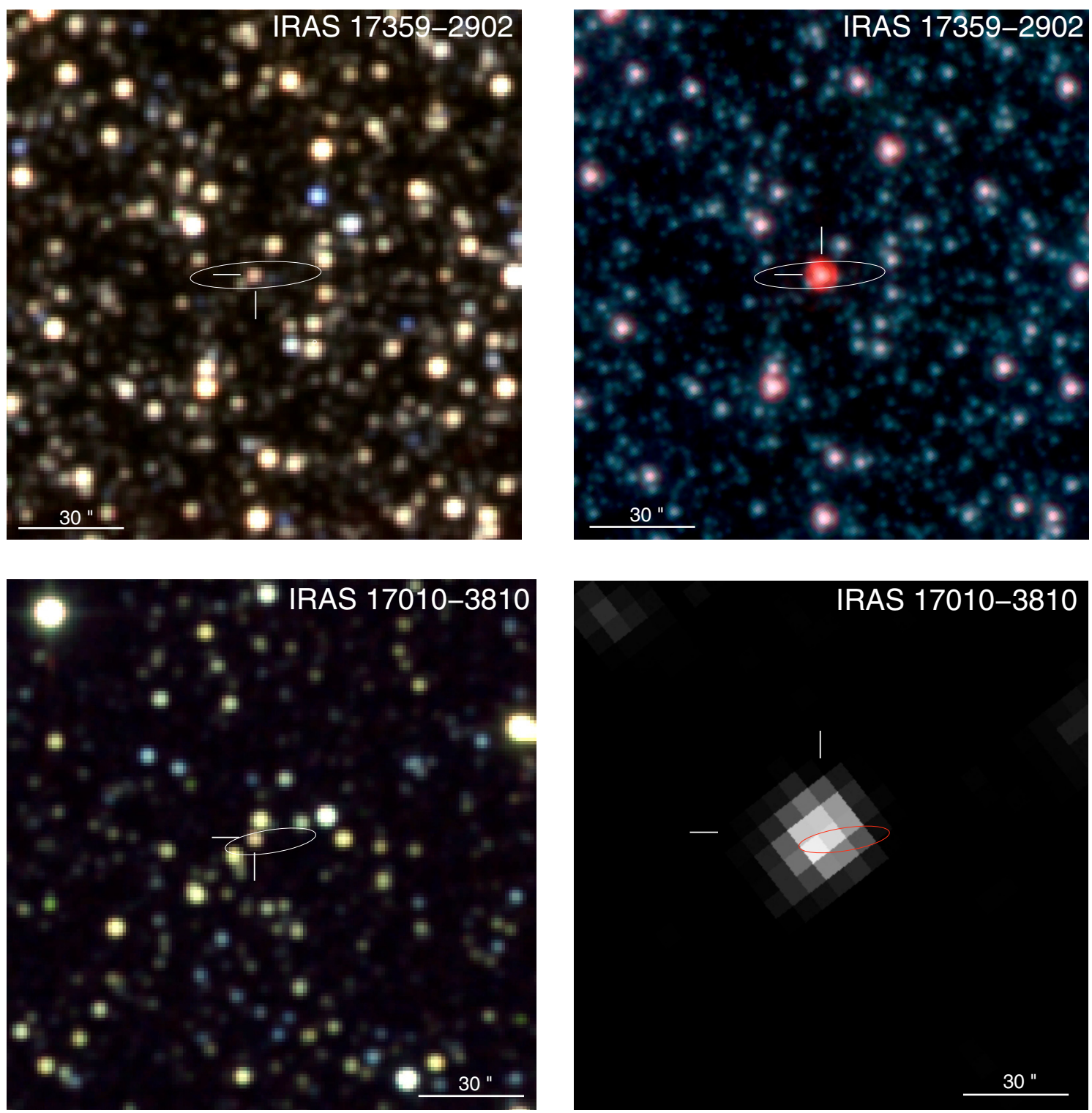

Fig. 2. Representative examples of objects whose near-IR counterparts have been identified using Spitzer GLIMPSE or MSX data. (top) 2 MASS $J$ (blue), $H$ (green), and $K_{\mathrm{S}}$ (red) composite picture (left) and Spitzer GLIMPSE $3.6 \mu \mathrm{m}$ (blue), $4.5 \mu \mathrm{m}$ (green), and $8.0 \mu \mathrm{m}$ (red) composite picture (right) of IRAS 17359-2902. (bottom) 2MASS $J$ (blue), $H$ (green), and $K_{\mathrm{S}}$ (red) composite picture (left) and MSX image in the $8.28 \mu \mathrm{m} A$ band (right) of IRAS 17010-3810. The locations of the near-IR counterparts and the IRAS ellipse-error are overlaid. North is up, east to the left.

\section{Discussion}

\subsection{Degree of obscuration among the IRAS sources}

Using the improved accuracy of the 2MASS coordinates, we searched for the optical counterpart of the sources in Tables 2 and 3 in the red plates of the Digitized Sky Survey ${ }^{1}$ (DSS). The

\footnotetext{
1 The Digitized Sky Survey is based on photographic data obtained using the UK Schmidt Telescope and the Oschin Schmidt Telescope on Palomar Mountain. The UK Schmidt was operated by the Royal Observatory of Edinburgh, with funding from the UK Science and Engineering Research Council, until 1988 June, and thereafter by the Anglo-Australian Observatory. The Palomar Observatory Sky Survey was funded by the National Geographic Society. The Oschin Schmidt Telescope is operated by the California Institute of Technology and Palomar Observatory. The plates were processed into the present compressed digital form with the permission of these institutes. The Digitized Sky Survey was produced at the Space Telescope Science Institute under US government grant NAGW-2166.
}

information on the optical detection of these sources, added to Tables 2 and 3, unveils a significant population of optical counterparts for the a priori supposedly heavily obscured IRAS postAGB and PN candidates. Out of the 119 objects with 2MASS counterpart, 59 have an optical counterpart, i.e., only $50 \%$ of the objects in these two tables can be considered optically obscured.

Despite the limited spatial resolution and sensitivity of the 2MASS images and, very notably, of the DSS red images, the homogeneity of these two databases grants a brightness-limited search that is meaningful in a statistical analysis. Therefore, the simple detection of an IRAS source in our sample in the DSS and/or 2MASS images allows us to determine the degree of obscuration of this source. Accordingly, we can define three types of sources based on the shortest wavelength at which they are detected:

- type $m$ sources (for mid-IR): heavily obscured sources that are detected neither in DSS red images nor in the 2MASS images; 
Table 2. IRAS post-AGB star and PN candidates with near-IR counterparts in the 2MASS PSC.

\begin{tabular}{|c|c|c|c|c|c|c|c|c|c|}
\hline Object & $\begin{array}{c}\alpha \\
(\mathrm{J} 2000)\end{array}$ & $\begin{array}{c}\delta \\
(\mathrm{J} 2000)\end{array}$ & $\begin{array}{l}\Delta \alpha \\
\left({ }^{\prime \prime}\right)\end{array}$ & $\begin{array}{l}\Delta \delta \\
\left({ }^{\prime \prime}\right)\end{array}$ & $\begin{array}{l}\text { Distance } \\
\left({ }^{\prime \prime}\right)\end{array}$ & Optical & $\begin{array}{c}J \\
(\mathrm{mag})\end{array}$ & $\begin{array}{c}H \\
(\mathrm{mag})\end{array}$ & $\begin{array}{c}K \\
(\mathrm{mag})\end{array}$ \\
\hline IRAS 00509+6623 & 005407.67 & +664012.8 & -0.2 & -0.2 & 0.3 & Yes & $13.950 \pm 0.032$ & $12.175 \pm 0.032$ & $10.634 \pm 0.024$ \\
\hline IRAS 08242-3828 & 082603.78 & -383847.4 & 3.3 & 0.6 & 3.3 & Yes & $7.08 \pm 0.04$ & $5.68 \pm 0.04$ & $4.912 \pm 0.022$ \\
\hline IRAS 09055-4629 & 090719.56 & -464123.6 & 1.6 & -2.6 & 3.1 & No & $15.90 \pm 0.08$ & $14.14 \pm 0.04$ & $12.686 \pm 0.032$ \\
\hline IRAS 09102-5101 & 091157.36 & $-51 \quad 1424.9$ & 36.3 & -33.9 & 49.6 & Yes & $14.41 \pm 0.05$ & $13.88 \pm 0.06$ & $12.77 \pm 0.05$ \\
\hline IRAS 09119-5150 & 091333.03 & -520241.6 & 1.2 & -2.6 & 2.9 & Yes & $13.99 \pm 0.04$ & $13.18 \pm 0.04$ & $11.85 \pm 0.04$ \\
\hline IRAS 09378-5117 & 093937.02 & -513129.3 & 1.1 & -3.3 & 3.5 & Yes & $12.077 \pm 0.026$ & $10.876 \pm 0.022$ & $10.273 \pm 0.021$ \\
\hline IRAS 09500-5236 & 095150.04 & -525052.1 & 4.9 & -12.1 & 13.1 & No & 17.2 & $14.62 \pm 0.06$ & $11.845 \pm 0.023$ \\
\hline IRAS 11339-6004 & 113620.69 & -60 2053.3 & -2.3 & 2.7 & 3.5 & No & $15.30 \pm 0.07$ & $14.20 \pm 0.07$ & $13.64 \pm 0.05$ \\
\hline IRAS 11488-6432 & 115117.36 & -64 4912.7 & 2.3 & -3.7 & 4.4 & Yes & $10.907 \pm 0.026$ & $9.354 \pm 0.024$ & $8.325 \pm 0.027$ \\
\hline IRAS 12262-6417 & 122904.49 & -643338.0 & 1.9 & -1.0 & 2.1 & Yes & $12.53 \pm 0.04$ & $10.754 \pm 0.029$ & $9.150 \pm 0.023$ \\
\hline IRAS 12309-5928 & 123344.58 & -594518.5 & -1.7 & -0.5 & 1.7 & No & $16.07 \pm 0.11$ & $13.843 \pm 0.030$ & $11.861 \pm 0.025$ \\
\hline IRAS 13356-6249 & 133906.31 & -630444.3 & 3.5 & -0.3 & 3.5 & No & $9.546 \pm 0.024$ & $8.00 \pm 0.06$ & $7.068 \pm 0.024$ \\
\hline IRAS 13398-5951 & 134312.56 & -600703.5 & 2.7 & -2.5 & 3.7 & No & $13.372 \pm 0.027$ & $11.813 \pm 0.023$ & $10.655 \pm 0.021$ \\
\hline IRAS 13421-6125 & 134534.08 & -614003.8 & -5.8 & 3.2 & 6.7 & Yes & $12.027 \pm 0.026$ & $10.835 \pm 0.022$ & $10.415 \pm 0.023$ \\
\hline IRAS 13483-5905 & 135143.73 & -59 2015.5 & -2.8 & 0.5 & 2.9 & Yes & $13.669 \pm 0.021$ & $12.800 \pm 0.022$ & $12.508 \pm 0.021$ \\
\hline IRAS 14104-5819 & 141400.56 & -583358.0 & 1.3 & -1.0 & 1.6 & No & 16.3 & $14.204 \pm 0.09$ & $10.690 \pm 0.024$ \\
\hline IRAS $14521-5300$ & 145545.70 & -531230.1 & -1.8 & 2.9 & 3.4 & Yes & $11.042 \pm 0.022$ & $10.236 \pm 0.023$ & $9.876 \pm 0.023$ \\
\hline IRAS $15038-5533$ & 150734.77 & -554450.7 & -5.3 & 3.3 & 6.3 & No & $15.04 \pm 0.07$ & $12.98 \pm 0.04$ & $11.784 \pm 0.027$ \\
\hline IRAS 15144-5812 & 151821.92 & -582311.9 & -0.6 & 1.1 & 1.3 & Yes & $11.066 \pm 0.026$ & $9.068 \pm 0.023$ & $7.348 \pm 0.018$ \\
\hline IRAS $15408-5657$ & 154448.36 & -570708.7 & 2.1 & -0.7 & 2.2 & No & 17.4 & 15.3 & $11.519 \pm 0.026$ \\
\hline IRAS 15408-5413 & 154439.80 & -542305.0 & -0.9 & 0.0 & 0.9 & No & $7.583 \pm 0.021$ & $4.769 \pm 0.015$ & $3.07 \pm 0.31$ \\
\hline IRAS $16228-5014$ & 162634.27 & -50 2101.8 & -20.4 & 3.2 & 20.6 & Yes & $9.927 \pm 0.026$ & $8.55 \pm 0.05$ & $7.735 \pm 0.023$ \\
\hline IRAS 16279-8158 & 163751.63 & -820449.9 & -1.0 & -0.9 & 1.3 & Yes & $10.308 \pm 0.023$ & $9.263 \pm 0.025$ & $8.818 \pm 0.023$ \\
\hline IRAS 16517-3626 & 165506.20 & -363132.2 & 13.3 & -2.2 & 13.4 & Yes & $12.652 \pm 0.026$ & $11.900 \pm 0.025$ & 11.2 \\
\hline IRAS 16559-2957 & 165908.22 & -300140.3 & 1.6 & -0.3 & 1.6 & Yes & $11.596 \pm 0.023$ & $10.713 \pm 0.023$ & $9.347 \pm 0.021$ \\
\hline IRAS 16567-3838 & 170009.07 & -384309.1 & 10.2 & -1.1 & 10.2 & No & 15.9 & 14.7 & $11.632 \pm 0.027$ \\
\hline IRAS $16584-3710$ & 170152.05 & -37 1453.7 & -5.4 & 3.3 & 6.3 & No & 16.5 & $14.00 \pm 0.04$ & $21 \pm 0.025$ \\
\hline IRAS 17010-3810 & 170427.31 & -38 1441.7 & 4.8 & 1.3 & 5.0 & Yes & $13.110 \pm 0.022$ & $12.040 \pm 0.028$ & $11.302 \pm($ \\
\hline IRAS 1702 & 170523.71 & -311318.7 & 6.5 & -0.7 & 6.6 & No & 17.4 & 16.4 & $13.88 \pm 0.05$ \\
\hline IRAS 17021-3054 & 170523.83 & -305813.0 & -3.5 & 0.0 & 3.5 & No & $14.61 \pm 0.08$ & $12.91 \pm 0.06$ & $11.532 \pm 0.034$ \\
\hline IRAS 17149-3053 & 171810.93 & -305639.9 & -9.9 & 0.1 & 9.9 & No & 15.8 & 14.5 & $13.37 \pm 0.06$ \\
\hline IRAS 17175-2819 & 171819.85 & -32 2721.6 & -0.9 & -1.4 & 1.7 & Yes & $11.10 \pm 0.04$ & $10.22 \pm 0.04$ & $9.391 \pm 0.024$ \\
\hline IRAS 17233-2602 & 172628.99 & -260456.8 & 3.9 & 1.2 & 4.1 & Yes & $14.93 \pm 0.04$ & $13.88 \pm 0.04$ & $13.10 \pm 0.04$ \\
\hline IRAS 17291-2147 & 173211.17 & -215002.2 & 14.9 & -3.2 & 15.2 & Yes & $13.507 \pm 0.021$ & $12.188 \pm 0.022$ & $11.008 \pm 0.019$ \\
\hline IRAS 17348-2906 & 173803.92 & -290816.5 & -3.7 & 6.5 & 7.5 & Yes & $8.027 \pm 0.023$ & $6.302 \pm 0.047$ & $5.395 \pm 0.018$ \\
\hline IRAS 17359-2902 & 173907.70 & -290402.8 & -0.1 & 0.1 & 0.2 & No & $12.75 \pm 0.06$ & $11.32 \pm 0.07$ & $10.44 \pm 0.04$ \\
\hline IRAS 17360-2142 & 173905.95 & -214351.4 & 0.7 & 0.6 & 0.9 & Yes & $10.825 \pm 0.023$ & $9.714 \pm 0.022$ & $9.107 \pm 0.019$ \\
\hline IRAS 17385-3332 & 174152.28 & -333340.6 & 1.0 & 0.4 & 1.1 & Yes & $10.484 \pm 0.029$ & $9.528 \pm 0.030$ & $9.018 \pm 0.029$ \\
\hline IRAS 17499-3520 & 175320.42 & $\begin{array}{lll}-35 & 21 & 10.3\end{array}$ & 8.8 & 0.7 & 8.8 & Yes & $10.663 \pm 0.024$ & $10.005 \pm 0.027$ & $9.595 \pm 0.019$ \\
\hline IRAS 17516-2525 & 175443.35 & -252628.0 & -2.0 & -1.0 & 2.3 & Yes & $8.695 \pm 0.020$ & $6.850 \pm 0.036$ & $5.082 \pm 0.016$ \\
\hline IRAS 17540-2753 & 175711.41 & -275419.0 & -35.7 & -3.0 & 35.8 & No & $12.326 \pm 0.028$ & $10.903 \pm 0.029$ & $9.996 \pm 0.021$ \\
\hline IRAS 17548-2753 & 175758.27 & -275320.2 & 7.6 & -0.2 & 7.6 & No & 15.3 & $12.78 \pm 0.04$ & $11.465 \pm 0.032$ \\
\hline IRAS $17550-2800$ & 175809.57 & -280025.3 & -13.6 & 0.7 & 13.7 & Yes & 12.5 & $11.04 \pm 0.06$ & 8.9 \\
\hline IRAS 17580-3111 & 180120.40 & -311120.3 & 9.0 & 2.7 & 9.4 & No & $12.402 \pm 0.024$ & $10.551 \pm 0.022$ & $9.280 \pm 0.019$ \\
\hline IRAS 17582-2619 & 180121.57 & -261937.3 & 5.0 & -0.3 & 5.0 & No & 15.3 & $12.527 \pm 0.030$ & $10.840 \pm 0.025$ \\
\hline IRAS 17596-3952 & 180306.77 & -395153.8 & 6.6 & 1.2 & 6.7 & Yes & $9.624 \pm 0.026$ & $8.845 \pm 0.026$ & $8.410 \pm 0.022$ \\
\hline IRAS 18015-1352 & 180422.66 & -135150.1 & 6.7 & -1.1 & 6.8 & No & 16.2 & 15.0 & $13.289 \pm 0.035$ \\
\hline IRAS 18049-2118 & 180755.12 & -211808.3 & 4.5 & 0.7 & 4.5 & No & 16.6 & 13.9 & $11.312 \pm 0.035$ \\
\hline IRAS 18071-1727 & 181006.07 & -172634.5 & 9.6 & 21.5 & 23.5 & No & 15.9 & 14.1 & $12.71 \pm 0.06$ \\
\hline IRAS 18105-1935 & 181329.92 & -193502.9 & -32.2 & 0.1 & 32.2 & No & 11.3 & $10.81 \pm 0.04$ & 9.2 \\
\hline IRAS 18113-2503 & 181427.26 & -250300.4 & 14.4 & -5.4 & 15.4 & Yes & $11.630 \pm 0.033$ & $10.698 \pm 0.034$ & $10.323 \pm 0.030$ \\
\hline IRAS 18183-2538 & 182124.49 & -253635.0 & -2.8 & 0.0 & 2.8 & Yes & $14.59 \pm 0.04$ & $13.043 \pm 0.026$ & $11.344 \pm 0.023$ \\
\hline IRAS 18199-1442 & 182250.92 & -144048.3 & 1.7 & 0.7 & 1.9 & No & 16.9 & 15.8 & $11.146 \pm 0.023$ \\
\hline IRAS 18229-1127 & 182541.49 & -112614.5 & -51.6 & -18.5 & 54.8 & Yes & $12.416 \pm 0.029$ & $11.39 \pm 0.04$ & $10.696 \pm 0.029$ \\
\hline IRAS 18236-0447 & 182619.56 & -044544.3 & -11.1 & -2.3 & 11.3 & No & 16.5 & $15.30 \pm 0.11$ & $13.10 \pm 0.04$ \\
\hline IRAS 18355-0712 & 183815.46 & -070954.3 & 0.9 & -2.3 & 2.5 & No & $15.85 \pm 0.11$ & $13.12 \pm 0.04$ & $11.528 \pm 0.035$ \\
\hline IRAS 18361-1203 & 183859.12 & -120042.0 & 4.7 & 2.0 & 5.1 & Yes & $11.727 \pm 0.026$ & $10.627 \pm 0.032$ & $10.027 \pm 0.024$ \\
\hline IRAS 18434-0042 & 184604.70 & -003854.4 & 4.5 & 0.6 & 4.5 & No & $16.13 \pm 0.10$ & $13.806 \pm 0.028$ & $12.573 \pm 0.022$ \\
\hline IRAS 18454+0001 & 184801.18 & +00 0448.5 & -4.8 & 1.5 & 5.0 & Yes & $14.061 \pm 0.026$ & $12.876 \pm 0.024$ & $12.112 \pm 0.025$ \\
\hline IRAS 18470+0015 & 184938.91 & +001854.9 & -2.9 & 2.9 & 4.1 & No & $14.07 \pm 0.13$ & $12.454 \pm 0.021$ & $10.765 \pm 0.020$ \\
\hline IRAS 18485+0642 & 185059.60 & +06 4559.0 & 10.4 & 4.0 & 11.2 & Yes & $14.020 \pm 0.030$ & $12.949 \pm 0.031$ & $12.325 \pm 0.029$ \\
\hline IRAS 18524+0544 & 185454.14 & +054811.2 & 0.0 & -0.1 & 0.1 & Yes & $14.58 \pm 0.04$ & $13.70 \pm 0.04$ & $12.412 \pm 0.033$ \\
\hline IRAS 18576+0341 & 190010.89 & +034547.1 & -0.2 & 0.1 & 0.2 & No & $12.164 \pm 0.027$ & $8.918 \pm 0.028$ & $7.007 \pm 0.020$ \\
\hline
\end{tabular}


Table 2. continued.

\begin{tabular}{cccrrclccc}
\hline \hline Object & $\begin{array}{c}\alpha \\
(\mathrm{J} 2000)\end{array}$ & $\begin{array}{c}\delta \\
(\mathrm{J} 2000)\end{array}$ & $\begin{array}{c}\Delta \alpha \\
\left({ }^{\prime \prime}\right)\end{array}$ & $\begin{array}{c}\Delta \delta \\
\left({ }^{\prime \prime}\right)\end{array}$ & $\begin{array}{c}\text { Distance } \\
\left({ }^{\prime \prime}\right)\end{array}$ & Optical & $\begin{array}{c}J \\
(\mathrm{mag})\end{array}$ & $\begin{array}{c}H \\
(\mathrm{mag})\end{array}$ & $\begin{array}{c}K \\
(\mathrm{mag})\end{array}$ \\
\hline IRAS 19006+1022 & 190300.03 & +102636.8 & 1.9 & 1.8 & 2.6 & No & $16.34 \pm 0.13$ & $13.55 \pm 0.04$ & $11.440 \pm 0.023$ \\
IRAS 19075+0432 & 190959.92 & +043708.5 & -1.2 & 2.5 & 2.8 & Yes & $10.973 \pm 0.021$ & $10.061 \pm 0.024$ & $8.745 \pm 0.025$ \\
IRAS 19079-0315 & 191032.66 & -031013.0 & 3.9 & 3.0 & 4.9 & Yes & $11.979 \pm 0.023$ & $11.205 \pm 0.025$ & $10.588 \pm 0.023$ \\
IRAS 19083+0119 & 191054.53 & +012445.0 & 1.9 & 3.0 & 3.6 & No & 16.9 & 15.5 & $14.34 \pm 0.08$ \\
IRAS 19094+1627 & 191144.68 & +163255.4 & 2.6 & 1.4 & 2.9 & Yes & $12.829 \pm 0.033$ & 11.8 & 11.1 \\
IRAS 19134+2131 & 191535.20 & +213634.0 & -2.8 & 1.0 & 3.0 & No & $16.54 \pm 0.13$ & $14.93 \pm 0.07$ & $13.46 \pm 0.04$ \\
IRAS 19176+1251 & 191955.77 & +125738.2 & -0.4 & 4.2 & 4.2 & No & $16.04 \pm 0.09$ & $14.52 \pm 0.05$ & $13.020 \pm 0.026$ \\
IRAS 19181+1806 & 192024.86 & +181141.4 & -4.8 & 0.4 & 4.9 & Yes & $13.472 \pm 0.025$ & $12.086 \pm 0.018$ & $10.984 \pm 0.020$ \\
IRAS 19193+1804 & 192131.63 & +181009.5 & 1.9 & 0.5 & 1.9 & No & 18.0 & $15.56 \pm 0.13$ & $14.29 \pm 0.07$ \\
IRAS 19319+2214 & 193403.58 & +222115.9 & 0.0 & -0.1 & 0.1 & No & $13.668 \pm 0.024$ & $12.003 \pm 0.022$ & $10.747 \pm 0.018$ \\
IRAS 19374+2359 & 193935.54 & +240627.0 & -0.8 & -1.0 & 1.3 & Yes & $12.038 \pm 0.025$ & $10.866 \pm 0.023$ & $9.735 \pm 0.017$ \\
IRAS 20174+3222 & 201927.81 & +323215.2 & -2.4 & 2.2 & 3.3 & Yes & $10.992 \pm 0.023$ & $10.118 \pm 0.032$ & $9.620 \pm 0.021$ \\
IRAS 20214+3749 & 202318.94 & +375851.6 & -3.1 & -0.4 & 3.1 & No & $16.96 \pm 0.20$ & $13.865 \pm 0.035$ & $11.046 \pm 0.018$ \\
IRAS 20244+3509 & 202625.16 & +351913.4 & -2.9 & -0.6 & 3.0 & Yes & $9.522 \pm 0.020$ & $8.188 \pm 0.029$ & $7.394 \pm 0.017$ \\
IRAS 20406+2953 & 204245.95 & +300406.4 & -0.2 & -0.1 & 0.2 & Yes & $10.966 \pm 0.021$ & $9.065 \pm 0.023$ & $7.920 \pm 0.021$ \\
IRAS 20461+3853 & 204804.43 & +390459.5 & -2.0 & -0.5 & 2.0 & Yes & $11.451 \pm 0.021$ & $10.294 \pm 0.019$ & $9.664 \pm 0.015$ \\
IRAS 21525+5643 & 215414.58 & +565727.0 & -3.4 & 4.0 & 5.3 & No & 16.5 & $16.00 \pm 0.16$ & $13.50 \pm 0.07$ \\
\hline
\end{tabular}

Table 3. IRAS post-AGB star and PN candidates with no counterparts in the 2MASS PSC.

\begin{tabular}{|c|c|c|}
\hline Object & Optical & Comments \\
\hline IRAS $04137+7016$ & Yes & Two objects unresolved by 2MASS. \\
\hline IRAS $05573+3156$ & No & Diffuse emission. \\
\hline IRAS 06499+0145 & Yes & Diffuse emission. \\
\hline IRAS 11381-6401 & Yes & Two objects unresolved by 2MASS. \\
\hline IRAS $11544-6408$ & Yes & Source very faint. 2MASS magnitudes not available. \\
\hline IRAS $12360-5740$ & Yes & 2MASS magnitudes contaminated by a nearby star. \\
\hline IRAS $12405-6219$ & No & Diffuse emission or multiple objects unresolved by $2 \mathrm{MASS}$. \\
\hline IRAS 13293-6000 & No & Two objects unresolved by 2MASS. \\
\hline IRAS $13427-6531$ & Yes & Multiple objects unresolved by 2 MASS. \\
\hline IRAS $13529-5934$ & Yes & Two objects unresolved by 2MASS. \\
\hline IRAS $14249-5310$ & No & Two objects unresolved by 2MASS. \\
\hline IRAS $15103-5754$ & No & Diffuse emission. \\
\hline IRAS $15229-5433$ & Yes & Diffuse emission or multiple objects unresolved by 2 MASS. \\
\hline IRAS $15452-5459$ & No & Diffuse emission. \\
\hline IRAS 15534-5422 & Yes & Diffuse emission. \\
\hline IRAS 16209-4714 & Yes & 2MASS magnitudes contaminated by a nearby star. \\
\hline IRAS 16296-4507 & Yes & Diffuse emission or two objects unresolved by 2 MASS. \\
\hline IRAS $16333-4807$ & No & Diffuse emission. \\
\hline IRAS $16507-4810$ & Yes & Diffuse emission or two objects unresolved by 2MASS. \\
\hline IRAS $17009-4154$ & No & Diffuse emission. \\
\hline IRAS $17052-3245$ & No & Source very faint. 2MASS magnitudes not available. \\
\hline IRAS $17150-3224$ & Yes & Diffuse emission, the Cotton Candy Nebula. \\
\hline IRAS $17234-4008$ & No & Two objects unresolved by 2MASS. \\
\hline IRAS 17269-2235 & Yes & Two objects unresolved by 2 MASS. \\
\hline IRAS $17301-2538$ & Yes & Two objects unresolved by 2MASS. \\
\hline IRAS 17361-4159 & No & Two objects unresolved by 2 MASS. \\
\hline IRAS 17376-3448 & No & Diffuse emission or multiple objects unresolved by 2 MASS. \\
\hline IRAS $17418-3335$ & No & Two objects unresolved by 2MASS. \\
\hline IRAS $17479-3032$ & No & Two objects unresolved by 2MASS. \\
\hline IRAS $17487-1922$ & Yes & Two objects unresolved by 2MASS. \\
\hline IRAS 17506-2955 & No & Two objects unresolved by 2MASS. \\
\hline IRAS 17560-2027 & Yes & Two objects unresolved by 2MASS. \\
\hline IRAS $18087-1440$ & No & Two objects unresolved by 2MASS. \\
\hline IRAS $19015+1256$ & No & Two objects unresolved by 2 MASS. \\
\hline IRAS $19071+0857$ & No & Diffuse emission or multiple objects unresolved by 2 MASS. \\
\hline IRAS $19178+1206$ & No & Two objects unresolved by 2MASS. \\
\hline IRAS $19208+1541$ & No & Two objects unresolved by 2 MASS. \\
\hline IRAS $20035+3242$ & No & Two objects unresolved by 2 MASS. \\
\hline IRAS 21554+6204 & No & Two objects unresolved by 2MASS. \\
\hline
\end{tabular}


Table 4. IRAS post-AGB star and PN candidates without 2MASS near-IR counterparts.

\begin{tabular}{l|l|l|l|l|l}
\hline \hline IRAS 08351-4634 & IRAS 15531-5704 & IRAS 17158-4049 & IRAS 17543-3102 & IRAS 18135-1456 & IRAS 19011+1049 \\
IRAS 09370-4826 & IRAS 16245-3859 & IRAS 17168-3736 & IRAS 17550-2120 & IRAS 18246-1032 & IRAS 19013+0629 \\
IRAS 10194-5625 & IRAS 16518-3425 & IRAS 17382-2531 & IRAS 17552-2030 & IRAS 18385+1350 & IRAS 19190+1102 \\
IRAS 11444-6150 & IRAS 16558-3417 & IRAS 17385-2413 & IRAS 18011-1847 & IRAS 18514+0019 & IRAS 19315+2235 \\
IRAS 11549-6225 & IRAS 17067-3759 & IRAS 17393-2727 & IRAS 18016-2743 & IRAS 18529+0210 & IRAS 19454+2920 \\
IRAS 13404-6059 & IRAS 17088-4221 & IRAS 17404-2713 & IRAS 18039-1903 & IRAS 18580+0818 & IRAS 20042+3259 \\
IRAS 13500-6106 & IRAS 17097-3624 & IRAS 17443-2949 & IRAS 18051-2415 & IRAS 18596+0315 & IRAS 21537+6435 \\
IRAS 15284-6026 & IRAS 17153-3814 & IRAS 17482-2501 & IRAS 18083-2155 & & \\
\hline
\end{tabular}
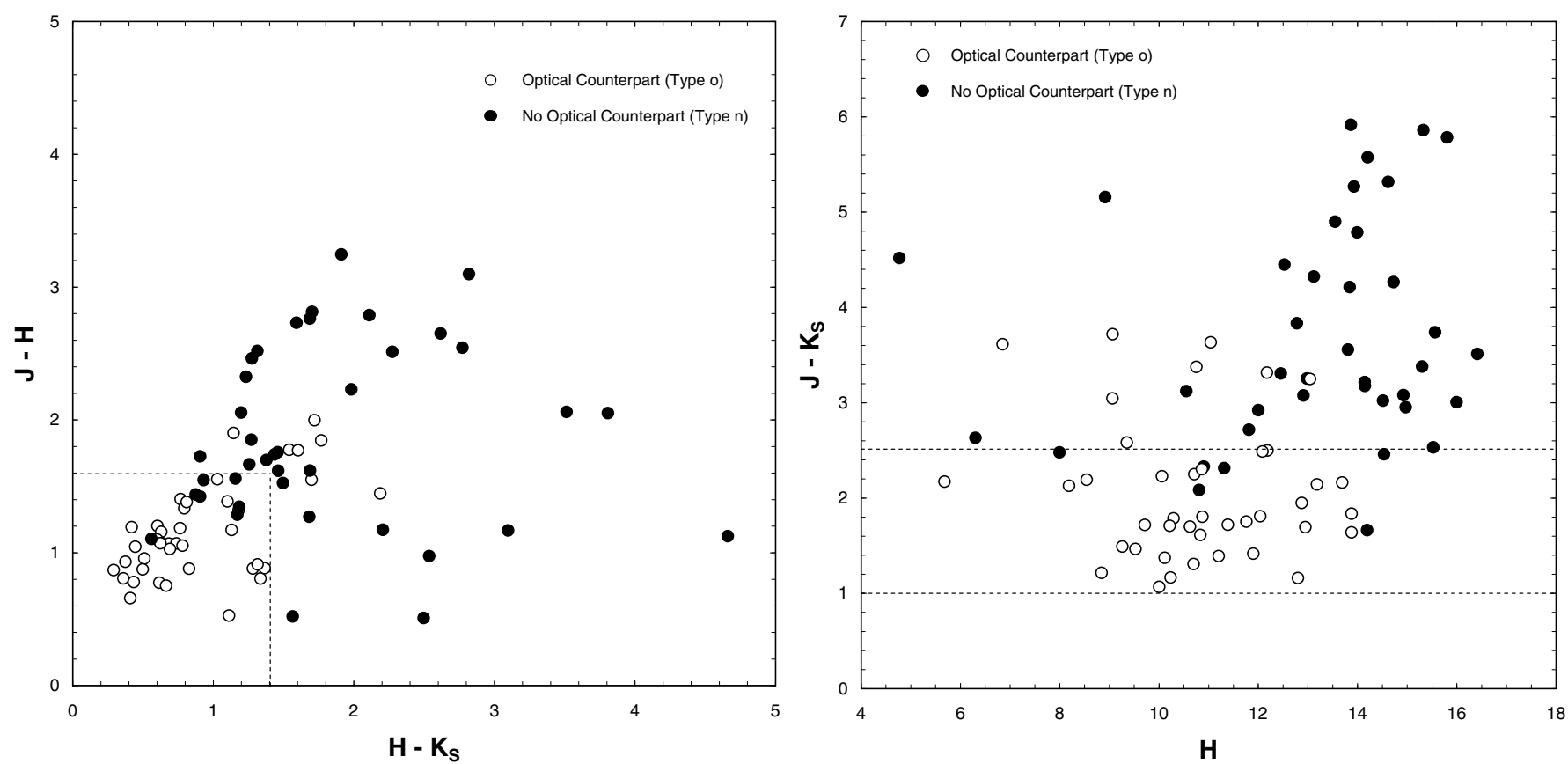

Fig. 5. (left) $J-H$ vs. $H-K_{\mathrm{s}}$ and (right) $J-K_{\mathrm{s}}$ vs. $H$ 2MASS diagrams of the IRAS post-AGB star and PN candidates in our sample with $2 \mathrm{MASS}$ PSC counterparts. The dashed lines mark the regions mostly occupied by sources of type $o$ (sources with optical counterpart).

- type $n$ sources (for near-IR): optically obscured sources detected in 2MASS images, but not in DSS images;

- type $o$ sources (for optical): sources with little optical and near-IR obscuration detected in both DSS and 2MASS images.

Type $m$ sources will be found among the sources listed in Table 4. The study of these sources will be postponed to an upcoming paper. Similarly, the 2MASS spatial resolution has not allowed us to measure the $J H K$ magnitudes of sources in Table 3 and study of these sources will also be deferred. In the following, we focus on the sources listed in Table 2, i.e., the sources of types $n$ and $o$ with a near-IR counterpart in the 2MASS PSC.

\subsection{Spectral properties}

The near- and mid-IR properties of the type $o$ and type $n$ sources can be used to investigate their nature. Using the JHK magnitudes of the sources listed in Table 2, the MSX and Spitzer GLIMPSE fluxes given in Table 5, and their IRAS fluxes (Table 1), we built the IR spectral energy distributions (SEDs) that are shown in Fig. 3. Analysis of the IR SEDs of these sources can be performed by studying the different color diagrams shown in Figs. 5-7.

The $J-H$ vs. $H-K_{\mathrm{s}}$ color diagram (Fig. 5) shows that the sources in this sample mostly have near-IR colors in the ranges $0.5<J-H<3$ and $0.2<H-K_{\mathrm{s}}<3$, although a few sources are very highly obscured, with $H-K_{\mathrm{s}}$ colors up to $\sim 5$ mag. In this diagram, sources detected in the optical tend to have lower values of their $J-H$ and $H-K_{\mathrm{s}}$ colors, with almost all these sources having $J-H<1.6$ and $H-K_{\mathrm{s}}<1.4$. This trend is more clearly seen in the $J-K_{\mathrm{s}}$ vs. $H$ diagram (Fig. 5) that shows noticeable differences in the distribution of sources of types $o$ and $n$. Type $o$ sources are mostly located on the band $1<J-K_{\mathrm{s}}<2.5$, while type $n$ sources are located mostly above this band, with $J-K_{\mathrm{s}} \geq 2.5$. Interpretation of this behavior is clearly illustrated by the SED of these two types of sources: type $o$ sources usually have flat or shallow near-IR SEDs (e.g., IRAS 14521-5300), while type $n$ sources present steep near-IR SEDs (e.g., IRAS 14104-5819).

The segregation between the sources of types $o$ and $n$ with a 2MASS PSC counterpart is also clear in the $M S X-J$ color diagram (Fig. 6). In this diagram, sources of type $o$ have $J-[8.3]$ colors typically below 10 . In contrast, sources of types $o$ and $n$ are not separated in the MSX color-color diagram (Fig. 6), where both types are spread over a wide region. Interestingly, these sources are mostly located in the quadrants of this diagram that have been suggested are populated by AGB and post-AGB stars, while generally avoiding the quadrant where objects in star-forming regions are typically found (Sevenster 2002). This result confirms our selection criteria for AGB and post-AGB stars in Sect. 2.

Similarly, the IRAS color-color diagram shown in Fig. 7 does not single out the optical type $o$ sources from the near-IR 
Table 5. Spitzer and MSX fluxes of IRAS post-AGB star and PN candidates with counterparts in the 2MASS PSC.

\begin{tabular}{|c|c|c|c|c|c|c|c|c|}
\hline & \multicolumn{4}{|c|}{ Spitzer } & \multicolumn{4}{|c|}{$M S X$} \\
\hline & $\begin{array}{c}3.6 \mu \mathrm{m} \\
{[\mathrm{Jy}]}\end{array}$ & $\begin{array}{c}4.5 \mu \mathrm{m} \\
{[\mathrm{Jy}]}\end{array}$ & $\begin{array}{c}5.8 \mu \mathrm{m} \\
{[\mathrm{Jy}]}\end{array}$ & $\begin{array}{c}8 \mu \mathrm{m} \\
{[\mathrm{Jy}]}\end{array}$ & $\begin{array}{c}8.28 \mu \mathrm{m} \\
{[\mathrm{Jy}]}\end{array}$ & $\begin{array}{c}12.13 \mu \mathrm{m} \\
{[\mathrm{Jy}]}\end{array}$ & $\begin{array}{c}14.65 \mu \mathrm{m} \\
{[\mathrm{Jy}]}\end{array}$ & $\begin{array}{c}21.3 \mu \mathrm{m} \\
{[\mathrm{Jy}]}\end{array}$ \\
\hline IRAS 00509+6623 & $\cdots$ & $\cdots$ & $\cdots$ & $\cdots$ & $1.18 \pm 0.05$ & $2.75 \pm 0.19$ & $2.45 \pm 0.18$ & $3.50 \pm 0.27$ \\
\hline IRAS 08242-3828 & $\ldots$ & $\ldots$ & $\ldots$ & $\ldots$ & $8.9 \pm 0.4$ & $11.3 \pm 0.6$ & $12.9 \pm 0.8$ & $20.7 \pm 1.2$ \\
\hline IRAS 09055-4629 & $\ldots$ & $\ldots$ & $\ldots$ & $\ldots$ & $0.26 \pm 0.01$ & $0.78 \pm 0.05$ & $0.86 \pm 0.06$ & $1.98 \pm 0.13$ \\
\hline IRAS 09102-5101 & $\ldots$ & $\ldots$ & $\ldots$ & $\ldots$ & $0.14 \pm 0.01$ & $\ldots$ & $1.06 \pm 0.08$ & $1.63 \pm 0.13$ \\
\hline IRAS 09119-5150 & $\ldots$ & $\ldots$ & $\ldots$ & $\ldots$ & $0.30 \pm 0.02$ & $1.05 \pm 0.09$ & $1.26 \pm 0.09$ & $2.14 \pm 0.16$ \\
\hline IRAS 09500-5236 & $\ldots$ & $\ldots$ & $\cdots$ & $\ldots$ & $0.23 \pm 0.01$ & $\ldots$ & $0.88 \pm 0.07$ & $\ldots$ \\
\hline IRAS 09378-5117 & $\ldots$ & $\cdots$ & $\cdots$ & $\ldots$ & $0.76 \pm 0.03$ & $2.17 \pm 0.13$ & $3.89 \pm 0.24$ & $4.92 \pm 0.31$ \\
\hline IRAS 11339-6004 & $\ldots$ & $\ldots$ & .. & $\ldots$ & $0.67 \pm 0.03$ & $2.37 \pm 0.14$ & $3.28 \pm 0.21$ & $5.65 \pm 0.35$ \\
\hline IRAS 11488-6432 & $\cdots$ & $\cdots$ & $\cdots$ & $\cdots$ & $1.03 \pm 0.04$ & $1.49 \pm 0.10$ & $1.72 \pm 0.11$ & $2.02 \pm 0.15$ \\
\hline IRAS 11544-6408 & $\ldots$ & $\cdots$ & $\cdots$ & $\cdots$ & $10.34 \pm 0.42$ & $24.6 \pm 1.2$ & $34.1 \pm 2.1$ & $34.0 \pm 2.0$ \\
\hline IRAS 12262-6417 & $\ldots$ & $\cdots$ & $\cdots$ & $\cdots$ & $1.86 \pm 0.08$ & $2.55 \pm 0.15$ & $2.64 \pm 0.17$ & $5.01 \pm 0.32$ \\
\hline IRAS 12309-5928 & $\cdots$ & $\cdots$ & $\cdots$ & $\ldots$ & $1.31 \pm 0.05$ & $2.00 \pm 0.12$ & $2.34 \pm 0.15$ & $3.90 \pm 0.25$ \\
\hline IRAS 13356-6249 & $\ldots$ & $\ldots$ & $0.747 \pm 0.022$ & $0.882 \pm 0.030$ & $1.49 \pm 0.06$ & $7.6 \pm 0.4$ & $22.1 \pm 1.4$ & $103 \pm 6$ \\
\hline IRAS 13398-5951 & $\ldots$ & $\cdots$ & $\ldots$ & $\ldots$ & $1.57 \pm 0.06$ & $2.70 \pm 0.15$ & $3.37 \pm 0.21$ & $4.81 \pm 0.30$ \\
\hline IRAS 13421-6125 & $0.273 \pm 0.031$ & $\cdots$ & $\cdots$ & $\cdots$ & $7.14 \pm 0.29$ & $14.6 \pm 0.7$ & $22.4 \pm 1.4$ & $22.0 \pm 1.3$ \\
\hline IRAS 13483-5905 & $\ldots$ & $\cdots$ & $\cdots$ & $\cdots$ & $1.07 \pm 0.04$ & $4.45 \pm 0.23$ & $10.0 \pm 0.6$ & $10.8 \pm 0.7$ \\
\hline IRAS 14104-5819 & $\ldots$ & $\ldots$ & $\ldots$ & $\ldots$ & $3.64 \pm 0.15$ & $4.59 \pm 0.24$ & $7.8 \pm 0.5$ & $9.2 \pm 0.6$ \\
\hline IRAS $15038-5533$ & $\ldots$ & $\ldots$ & $\ldots$ & $\ldots$ & $0.63 \pm 0.03$ & $1.25 \pm 0.09$ & $1.61 \pm 0.11$ & $2.15 \pm 0.16$ \\
\hline IRAS 15144-5812 & $0.35 \pm 0.07$ & $3.35 \pm 0.21$ & $4.41 \pm 0.11$ & $\ldots$ & $5.13 \pm 0.21$ & $6.53 \pm 0.33$ & $8.8 \pm 0.5$ & $26.6 \pm 1.6$ \\
\hline IRAS $15408-5657$ & $\ldots$ & $\ldots$ & $\ldots$ & $\ldots$ & $65.9 \pm 2.7$ & $102 \pm 5$ & $112 \pm 7$ & $123 \pm 7$ \\
\hline IRAS $15408-5413$ & $\ldots$ & $\cdots$ & $\cdots$ & $\cdots$ & $135 \pm 6$ & $231 \pm 12$ & $257 \pm 16$ & $298 \pm 18$ \\
\hline IRAS $16228-5014$ & $\ldots$ & $\ldots$ & $0.690 \pm 0.020$ & $0.463 \pm 0.011$ & $0.22 \pm 0.01$ & $0.76 \pm 0.07$ & $1.35 \pm 0.09$ & $11.4 \pm 0.7$ \\
\hline IRAS 16517-3626 & $\cdots$ & $\cdots$ & $\ldots$ & $\ldots$ & $0.75 \pm 0.03$ & $1.65 \pm 0.13$ & $2.39 \pm 0.16$ & $5.45 \pm 0.35$ \\
\hline IRAS 16518-3425 & $\cdots$ & $\cdots$ & $\cdots$ & $\cdots$ & $0.35 \pm 0.02$ & $\ldots$ & $1.95 \pm 0.14$ & $6.7 \pm 0.4$ \\
\hline IRAS 16567-3838 & $\cdots$ & $\cdots$ & $\cdots$ & $\cdots$ & $5.88 \pm 0.24$ & $8.5 \pm 0.4$ & $11.1 \pm 0.7$ & $13.4 \pm 0.8$ \\
\hline IRAS 16584-3710 & $\cdots$ & $\cdots$ & $\cdots$ & $\cdots$ & $0.43 \pm 0.02$ & $0.95 \pm 0.10$ & $1.96 \pm 0.13$ & $4.00 \pm 0.26$ \\
\hline IRAS 17010-3810 & $\cdots$ & $\cdots$ & $\cdots$ & $\cdots$ & $0.55 \pm 0.02$ & $2.21 \pm 0.13$ & $4.08 \pm 0.25$ & $8.2 \pm 0.5$ \\
\hline IRAS 17021-3109 & $\ldots$ & $\ldots$ & $\cdots$ & $\cdots$ & $1.06 \pm 0.05$ & $2.99 \pm 0.21$ & $6.3 \pm 0.4$ & $9.1 \pm 0.6$ \\
\hline IRAS 17021-3054 & $\cdots$ & $\cdots$ & $\cdots$ & $\cdots$ & $0.42 \pm 0.02$ & $\ldots$ & $3.32 \pm 0.23$ & $4.51 \pm 0.32$ \\
\hline IRAS 17149-3053 & $\cdots$ & $\cdots$ & $\cdots$ & $\cdots$ & $0.41 \pm 0.02$ & $2.02 \pm 0.13$ & $3.93 \pm 0.25$ & $8.2 \pm 0.5$ \\
\hline IRAS 17175-2819 & $\cdots$ & $\cdots$ & $\cdots$ & $\cdots$ & $12.7 \pm 0.5$ & $61 \pm 3$ & $128 \pm 8$ & $247 \pm 15$ \\
\hline IRAS 17233-2602 & $\cdots$ & $\cdots$ & $\cdots$ & $\cdots$ & $1.94 \pm 0.08$ & $4.32 \pm 0.23$ & $7.4 \pm 0.5$ & $7.9 \pm 0.5$ \\
\hline IRAS 17348-2906 & $\ldots$ & $\ldots$ & $2.54 \pm 0.07$ & $\ldots$ & $1.92 \pm 0.08$ & $3.30 \pm 0.18$ & $3.92 \pm 0.24$ & $7.7 \pm 0.5$ \\
\hline IRAS 17359-2902 & $0.0520 \pm 0.0020$ & $0.0522 \pm 0.0017$ & $0.0610 \pm 0.0024$ & $0.261 \pm 0.007$ & $0.44 \pm 0.02$ & $1.70 \pm 0.11$ & $3.27 \pm 0.21$ & $10.0 \pm 0.6$ \\
\hline IRAS 17360-2142 & $\ldots$ & $\ldots$ & $\ldots$ & $\ldots$ & $0.26 \pm 0.01$ & $1.3 \pm 0.6$ & $2.31 \pm 0.15$ & $8.9 \pm 0.5$ \\
\hline IRAS 17385-3332 & $\cdots$ & $\cdots$ & $\cdots$ & $\cdots$ & $0.19 \pm 0.01$ & $0.73 \pm 0.08$ & $1.78 \pm 0.12$ & $7.7 \pm 0.5$ \\
\hline IRAS 17499-3520 & $\cdots$ & $\cdots$ & $\cdots$ & $\cdots$ & $0.76 \pm 0.03$ & $2.40 \pm 0.15$ & $3.65 \pm 0.23$ & $10.3 \pm 0.6$ \\
\hline IRAS 17516-2525 & $\cdots$ & $\cdots$ & $\ldots$ & $\ldots$ & $40.5 \pm 1.7$ & $43.9 \pm 2.2$ & $44.7 \pm 2.7$ & $89 \pm 5$ \\
\hline IRAS 17540-2753 & $0.0590 \pm 0.0020$ & $0.0483 \pm 0.0027$ & $0.0580 \pm 0.0018$ & $0.292 \pm 0.010$ & $0.38 \pm 0.02$ & $1.41 \pm 0.10$ & $3.74 \pm 0.24$ & $10.0 \pm 0.6$ \\
\hline IRAS 17548-2753 & $0.0367 \pm 0.0015$ & $0.0462 \pm 0.0019$ & $0.0480 \pm 0.0014$ & $0.136 \pm 0.004$ & $0.23 \pm 0.01$ & $1.00 \pm 0.09$ & $3.60 \pm 0.23$ & $11.4 \pm 0.7$ \\
\hline IRAS 17550-2800 & $\ldots$ & $\ldots$ & $1.90 \pm 0.06$ & $\ldots$ & $2.29 \pm 0.09$ & $2.17 \pm 0.13$ & $3.00 \pm 0.19$ & $4.28 \pm 0.28$ \\
\hline IRAS 17580-3111 & $\cdots$ & $\cdots$ & $\ldots$ & $\cdots$ & $1.24 \pm 0.05$ & $3.62 \pm 0.18$ & $6.9 \pm 0.4$ & $13.2 \pm 0.8$ \\
\hline IRAS 17582-2619 & $\cdots$ & $\cdots$ & $\cdots$ & $\cdots$ & $0.38 \pm 0.02$ & $1.65 \pm 0.11$ & $3.45 \pm 0.22$ & $7.0 \pm 0.4$ \\
\hline IRAS 18015-1352 & $\ldots$ & $\ldots$ & $\ldots$ & $\cdots$ & $1.46 \pm 0.06$ & $2.39 \pm 0.14$ & $2.48 \pm 0.16$ & $3.42 \pm 0.22$ \\
\hline IRAS 18049-2118 & $\cdots$ & $3.90 \pm 0.34$ & $11.4 \pm 0.8$ & $\cdots$ & $8.31 \pm 0.34$ & $13.4 \pm 0.7$ & $17.6 \pm 1.1$ & $18.3 \pm 1.1$ \\
\hline IRAS 18071-1727 & $0.147 \pm 0.006$ & $\ldots$ & $\ldots$ & $\ldots$ & $8.22 \pm 0.34$ & $28.7 \pm 1.4$ & $51.5 \pm 3.1$ & $57.5 \pm 3.5$ \\
\hline IRAS 18105-1935 & $0.370 \pm 0.021$ & $\cdots$ & $2.22 \pm 0.05$ & $4.32 \pm 0.11$ & $3.78 \pm 0.15$ & $8.3 \pm 0.4$ & $12.0 \pm 0.7$ & $15.0 \pm 0.9$ \\
\hline IRAS 18113-2503 & $\ldots$ & $\ldots$ & $\ldots$ & $\ldots$ & $0.69 \pm 0.03$ & $2.53 \pm 0.14$ & $5.48 \pm 0.33$ & $9.6 \pm 0.6$ \\
\hline IRAS 18199-1442 & $0.542 \pm 0.04$ & $2.83 \pm 0.15$ & $\cdots$ & $\cdots$ & $15.7 \pm 0.6$ & $22.3 \pm 1.1$ & $29.8 \pm 1.8$ & $28.4 \pm 1.7$ \\
\hline IRAS $18229-1127$ & $0.0368 \pm 0.0029$ & $0.0434 \pm 0.0024$ & $0.161 \pm 0.007$ & $0.515 \pm 0.04$ & $0.50 \pm 0.02$ & $1.18 \pm 0.09$ & $1.92 \pm 0.12$ & $17.4 \pm 1.1$ \\
\hline IRAS 18236-0447 & $\ldots$ & $\ldots$ & $\ldots$ & $\ldots$ & $0.67 \pm 0.03$ & $1.40 \pm 0.10$ & $2.70 \pm 0.17$ & $2.73 \pm 0.19$ \\
\hline IRAS 18355-0712 & $0.0416 \pm 0.0019$ & $0.0689 \pm 0.0019$ & $0.1011 \pm 0.0021$ & $0.227 \pm 0.004$ & $0.27 \pm 0.01$ & $1.55 \pm 0.08$ & $3.86 \pm 0.24$ & $9.4 \pm 0.6$ \\
\hline IRAS 18361-1203 & $\ldots$ & $\ldots$ & $\ldots$ & $\ldots$ & $1.57 \pm 0.06$ & $2.63 \pm 0.15$ & $3.29 \pm 0.21$ & $4.31 \pm 0.27$ \\
\hline IRAS 18434-0042 & $0.0078 \pm 0.0004$ & $0.0113 \pm 0.0007$ & $0.0266 \pm 0.0010$ & $0.1249 \pm 0.0031$ & $0.14 \pm 0.01$ & $0.62 \pm 0.07$ & $1.48 \pm 0.10$ & $2.81 \pm 0.18$ \\
\hline IRAS 18454+0001 & $0.0105 \pm 0.0004$ & $0.0106 \pm 0.0003$ & $0.0319 \pm 0.0009$ & $0.099 \pm 0.004$ & $0.12 \pm 0.01$ & $0.41 \pm 0.03$ & $1.24 \pm 0.08$ & $9.5 \pm 0.6$ \\
\hline IRAS $18470+0015$ & $0.216 \pm 0.009$ & $0.447 \pm 0.018$ & $0.807 \pm 0.022$ & $\ldots$ & $2.15 \pm 0.09$ & $4.64 \pm 0.23$ & $6.1 \pm 0.4$ & $8.8 \pm 0.5$ \\
\hline IRAS $18485+0642$ & $\ldots$ & $\ldots$ & $\ldots$ & $\cdots$ & $0.99 \pm 0.04$ & $3.42 \pm 0.18$ & $7.8 \pm 0.5$ & $14.9 \pm 0.9$ \\
\hline IRAS 18524+0544 & $\cdots$ & $\cdots$ & $\cdots$ & $\cdots$ & $0.19 \pm 0.01$ & $\ldots$ & $1.44 \pm 0.10$ & $3.43 \pm 0.22$ \\
\hline IRAS 18576+0341 & $2.3 \pm 0.4$ & $\cdots$ & $\cdots$ & $\cdots$ & $10.6 \pm 0.4$ & $45.5 \pm 2.3$ & $89 \pm 5$ & $277 \pm 17$ \\
\hline IRAS $19006+1022$ & $\cdots$ & $\cdots$ & $\cdots$ & $\cdots$ & $0.56 \pm 0.02$ & $\cdots$ & $1.47 \pm 0.10$ & $2.93 \pm 0.20$ \\
\hline IRAS 19075+0432 & $\cdots$ & $\cdots$ & $\cdots$ & $\cdots$ & $4.51 \pm 0.19$ & $5.34 \pm 0.27$ & $8.6 \pm 0.5$ & $17.0 \pm 1.0$ \\
\hline
\end{tabular}


Table 5. continued.

\begin{tabular}{|c|c|c|c|c|c|c|c|c|}
\hline & \multicolumn{4}{|c|}{ Spitzer } & \multicolumn{4}{|c|}{$\overline{M S X}$} \\
\hline & $\begin{array}{c}3.6 \mu \mathrm{m} \\
{[\mathrm{Jy}]}\end{array}$ & $\begin{array}{c}4.5 \mu \mathrm{m} \\
{[\mathrm{Jy}]}\end{array}$ & $\begin{array}{c}5.8 \mu \mathrm{m} \\
{[\mathrm{Jy}]}\end{array}$ & $\begin{array}{c}8 \mu \mathrm{m} \\
{[\mathrm{Jy}]}\end{array}$ & $\begin{array}{c}8.28 \mu \mathrm{m} \\
{[\mathrm{Jy}]}\end{array}$ & $\begin{array}{c}12.13 \mu \mathrm{m} \\
{[\mathrm{Jy}]}\end{array}$ & $\begin{array}{c}14.65 \mu \mathrm{m} \\
{[\mathrm{Jy}]}\end{array}$ & $\begin{array}{c}21.3 \mu \mathrm{m} \\
{[\mathrm{Jy}]}\end{array}$ \\
\hline IRAS $19083+0119$ & $\cdots$ & $\cdots$ & $\cdots$ & $\cdots$ & $0.54 \pm 0.02$ & $2.18 \pm 0.13$ & $5.08 \pm 0.31$ & $11.1 \pm 0.7$ \\
\hline IRAS 19094+1627 & $\ldots$ & $\ldots$ & $\ldots$ & $\cdots$ & $0.32 \pm 0.02$ & $0.75 \pm 0.08$ & $1.34 \pm 0.10$ & $2.40 \pm 0.17$ \\
\hline IRAS 19134+2131 & $\ldots$ & $\ldots$ & $\ldots$ & $\ldots$ & $1.22 \pm 0.05$ & $5.06 \pm 0.27$ & $8.9 \pm 0.5$ & $10.1 \pm 0.6$ \\
\hline IRAS $19176+1251$ & $0.0071 \pm 0.0003$ & $0.0124 \pm 0.0005$ & $0.0189 \pm 0.0006$ & $0.162 \pm 0.005$ & $0.32 \pm 0.01$ & $1.13 \pm 0.08$ & $2.06 \pm 0.13$ & $8.1 \pm 0.5$ \\
\hline IRAS $19181+1806$ & $\ldots$ & $\ldots$ & $\ldots$ & $\ldots$ & $0.22 \pm 0.01$ & $\ldots$ & $0.80 \pm 0.07$ & $2.63 \pm 0.18$ \\
\hline IRAS $19193+1804$ & $\ldots$ & $\ldots$ & $\ldots$ & $\ldots$ & $0.11 \pm 0.01$ & $\ldots$ & $1.95 \pm 0.13$ & $5.17 \pm 0.32$ \\
\hline IRAS $19319+2214$ & $\ldots$ & $\ldots$ & $\ldots$ & $\ldots$ & $2.23 \pm 0.09$ & $3.52 \pm 0.19$ & $4.82 \pm 0.30$ & $5.27 \pm 0.33$ \\
\hline IRAS $19374+2359$ & $0.430 \pm 0.011$ & $\ldots$ & .. & .. & $13.8 \pm 0.6$ & $23.72 \pm 1.19$ & $33.7 \pm 2.1$ & $68 \pm 4$ \\
\hline IRAS $20174+3222$ & $\ldots$ & $\ldots$ & $\ldots$ & $\ldots$ & $0.87 \pm 0.04$ & $2.81 \pm 0.16$ & $3.32 \pm 0.21$ & $11.5 \pm 0.7$ \\
\hline IRAS 20214+3749 & $\ldots$ & $\ldots$ & $\ldots$ & $\cdots$ & $1.23 \pm 0.05$ & $1.93 \pm 0.12$ & $2.18 \pm 0.14$ & $2.60 \pm 0.18$ \\
\hline IRAS $20244+3509$ & $\ldots$ & $\ldots$ & $\ldots$ & $\ldots$ & $0.76 \pm 0.03$ & $1.08 \pm 0.09$ & $1.53 \pm 0.11$ & $3.03 \pm 0.20$ \\
\hline IRAS $20461+3853$ & $\ldots$ & $\ldots$ & $\ldots$ & $\ldots$ & $0.82 \pm 0.03$ & $2.48 \pm 0.14$ & $3.01 \pm 0.19$ & $6.8 \pm 0.4$ \\
\hline IRAS $21525+5643$ & $\ldots$ & $\ldots$ & $\ldots$ & $\ldots$ & $2.28 \pm 0.09$ & $5.25 \pm 0.27$ & $6.8 \pm 0.4$ & $9.9 \pm 0.6$ \\
\hline
\end{tabular}
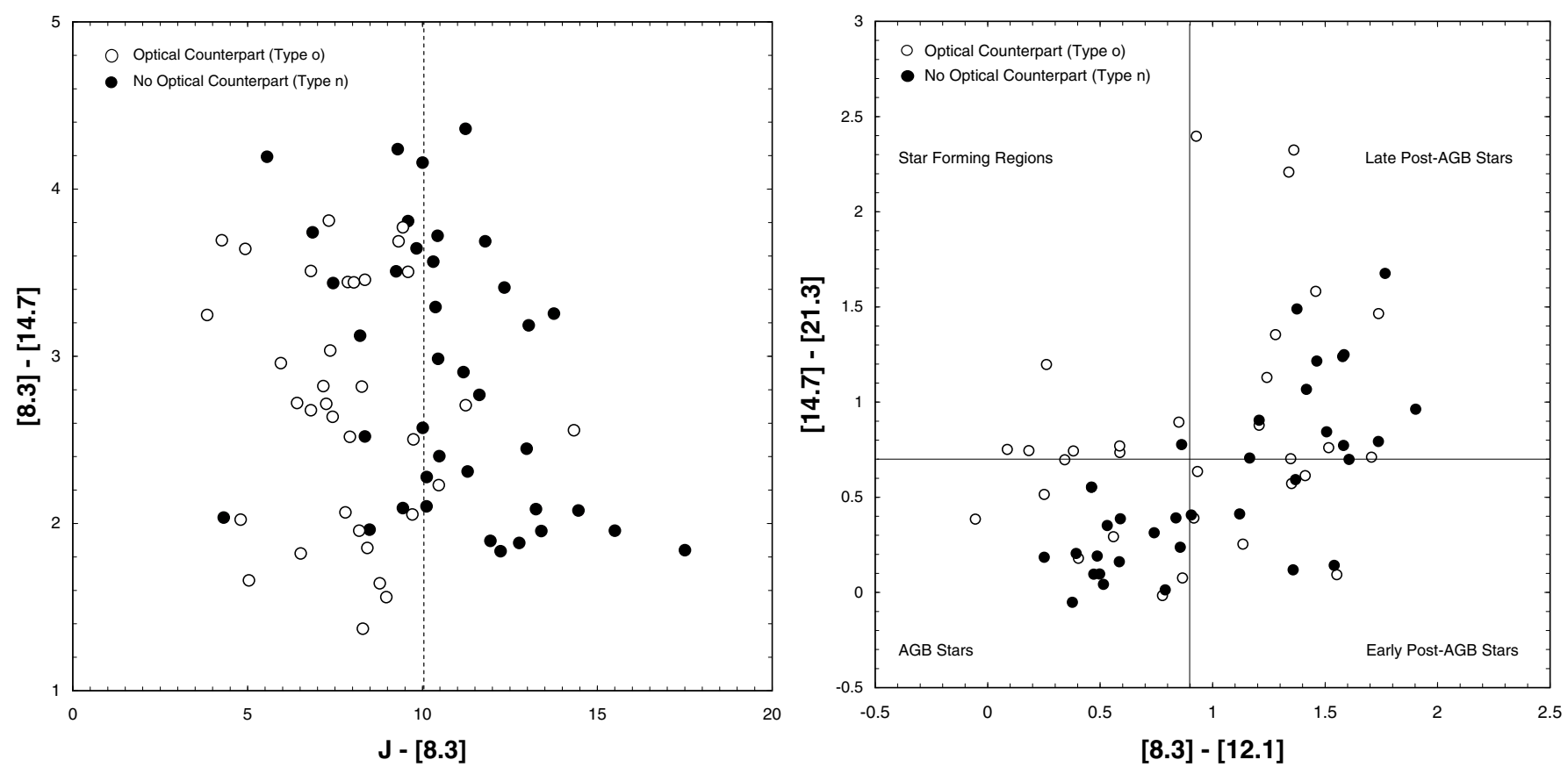

Fig. 6. (left) $M S X-J$ and (right) $M S X$ color-color diagrams of the IRAS post-AGB star and PN candidates with 2 MASS PSC and $M S X$ counterparts. The vertical line in the $M S X-J$ color-color diagram at $J-[8.3]=10$ marks a rough division between sources of types $o$ and $n$. The vertical and horizontal lines in the $M S X$ color-color diagram divide it into four quadrants that correspond to areas of higher probability of containing the different types of objects labeled on the diagram (Sevenster 2002).

type $n$ sources in Table 2. On the other hand, the two types of sources show different properties in the $K_{\mathrm{s}}-$ [8.3] vs. $J-$ [25] color-color diagram (Fig. 7). In this diagram (spotlighted by Suárez et al., in prep., as able to distinguish very efficiently between different types of SEDs of post-AGB stars), sources of type $o$ have mostly $J-[25]<7$ and sources of type $n$ have $J-[25]>9.6$, with an intermediate region populated by both types $o$ and $n$ sources. It is interesting to note that the $K_{\mathrm{s}}-[8.3]$ and $J-[25]$ colors of the sources in our sample are clearly correlated.

In all the color-color diagrams shown in Figs. 5-7, the use of colors in which the $J$ magnitude is involved has allowed us to separate the sources with optical counterparts from these obscured in the optical. It can be concluded that the relation between the magnitude in the $J$ band and in different bands in the near-, mid-, and far-IR is critical in forecasting the level of emission at optical wavelenghts.

\subsection{Sources in the Spitzer GLIMPSE Image Atlas}

The Spitzer images of the IRAS post-AGB star and PN candidates with 2MASS PSC counterparts that are available in the GLIMPSE Image Atlas are presented in Fig. 8 (which is at the end of the text). All IRAS sources identified in the Spitzer GLIMPSE Image Atlas are point-like, except IRAS 18229-1127, IRAS 18454+0001, and IRAS 18576+0341, whose PSFs have FWHMs broader than those of point sources in their respective images. IRAS 18229-1127 has an FWHM of $3{ }^{\prime \prime} 0$ in the 4 IRAC bands, just above the FWHM of stellar sources $(\sim 2$.' 1$)$. IRAS $18454+0001$ is a point-like source, except in the $8 \mu \mathrm{m}$ band, with an FWHM of 2". 4 (compared to the FWHM of 1'.9 of stellar sources). Finally, IRAS 18576+0341 has the broadest FWHM $\left(\sim 5^{\prime \prime} .0\right)$ in the $3.6 \mu \mathrm{m}, 4.5 \mu \mathrm{m}$, and $5.8 \mu \mathrm{m}$ bands (compared to the FWHM of $\sim 2$ '. 0 of stellar sources). In the $8 \mu \mathrm{m}$ band, its extension is unknown, as its image in this band is saturated. 

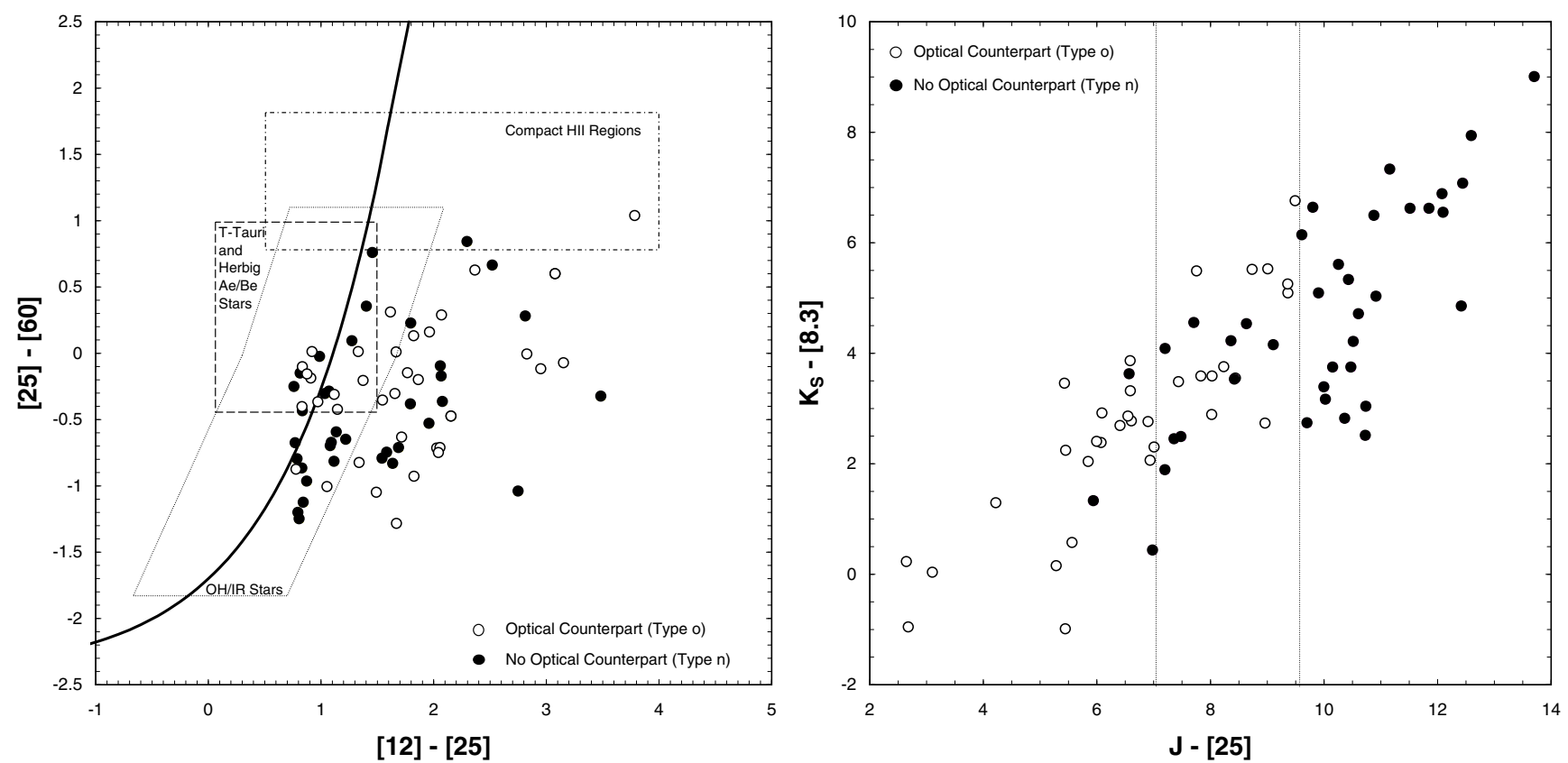

Fig. 7. (left) IRAS and (right) $K_{\mathrm{S}}-$ [8.3] vs. $J-$ [25] color-color diagrams of the IRAS post-AGB star and PN candidates with 2MASS PSC and MSX counterparts. In the IRAS color-color diagram, the locii of OH/IR stars, T-Tauri and Herbig Ae/Be stars, and compact $\mathrm{H}$ II are overlaid as in Fig. 1. The vertical lines in the $K_{\mathrm{s}}-[8.3]$ vs. $J-$ [25] diagram mark three regions with the leftmost being dominated by sources with optical counterpart (type $o$ sources), and the rightmost being exclusively populated by sources with no optical counterpart (type $n$ sources).

\section{Summary}

Using 2MASS, Spitzer GLIMPSE, and MSX data products, we have identified 119 near-IR counterparts out of a sample of 165 IRAS post-AGB star and PN candidates. For these sources, we provide near-IR identification charts. Among those 119 sources with near-IR counterparts, 80 objects have unambiguous 2MASS PSC counterparts. For these sources, we further provide improved coordinates and IR energy spectral distributions from $1 \mu \mathrm{m}$ to $100 \mu \mathrm{m}$.

Using the improved coordinates of the sources with 2MASS PSC counterpart, we searched for their optical counterparts in DSS red plates and found that $\sim 50 \%$ of these sources are detected in the optical. We then investigated the different spectral properties of the sources with and without optical counterparts, dubbed sources of types $o$ and $n$, respectively. The two types of sources have similar mid- and far-IR colors, but they appear clearly segregated in color-color diagrams in which the magnitude in the $J$ band is used to compute one of the colors.

Acknowledgements. This publication makes use of data products from the 2MASS, which is a joint project of the University of Massachusetts and the Infrared Processing and Analysis Center/California Institute of Technology, funded by the National Aeronautics and Space Administration and the National Science Foundation. This work is based, in part, on observations made with the Spitzer Space Telescope, which is operated by the Jet Propulsion Laboratory, California Institute of Technology under a contract with NASA. Support for this work was provided by an award issued by JPL/Caltech. G.R.L. acknowledges support of a postdoctoral fellowship from CONACyT (Mexico) grant 75861. M.A.G., O.S., L.F.M., and J.F.G. acknowledge support from Consejería de Innovación, Ciencia y Empresa of Junta de Andalucía. G.R.L., M.A.G., and L.F.M. are partially funded by grant AYA2005-01495 of the Spanish Ministerio de Educación y Ciencia (MEC). J.F.G. is partially funded by grant AYA200508523-C03-03 of MEC. G.R.L., M.A.G., O.S., and L.F.M. are partially funded by grant AYA2008-01934 of the Spanish Ministerio de Ciencia e Innovación (MICINN). O.S. and J.F.G. are partially funded by grant AYA2008-06189-C0301 of MICINN. This research has made use of the SIMBAD database, operated at CDS, Strasbourg, France.

\section{References}

Antonopoulos, E., \& Pottasch, S. R. 1987, A\&A, 173, 108

Bedijn, P. J. 1987, A\&A, 186, 136

Beichman, C. A., Neugebauer, G., Habing, H. J., Clegg, P. E., \& Chester, T. J. 1988, Infrared astronomical satellite (IRAS) catalogs and atlases, Explanatory supplement, 1, 1

Benjamin, R. A., Churchwell, E., Babler, B. L., et al. 2003, PASP, 115, 953

Bloecker, T. 1995, A\&A, 299, 755

Fazio, G. G., Hora, J. L., Allen, L. E., et al. 2004, ApJS, 154, 10

García-Lario, P., Manchado, A., Suso, S. R., Pottasch, S. R., \& Olling, R. 1990, A\&AS, 82, 497

García-Lario, P., Manchado, A., Pych, W., \& Pottasch, S. R. 1997, A\&AS, 126, 479

Harris, S., Clegg, P., \& Hughes, J. 1988, MNRAS, 235, 441

Hrivnak, B. J., Kelly, D. M., Su, K. Y. L., Kwok, S., \& Sahai, R. 2006, ApJ, 650, 237

Hu, J. Y., Slijkhuis, S., de Jong, T., \& Jiang, B. W. 1993, A\&AS, 100, 413

Kenyon, S. J., \& Hartmann, L. 1995, ApJS, 101, 117

Kwok, S., Su, K. Y. L., \& Hrivnak, B. J. 1998, ApJ, 501, L117

Malfait, K., Bogaert, E., \& Waelkens, C. 1998, A\&A, 331, 211

Meeus, G., Waters, L. B. F. M., Bouwman, J., et al. 2001, A\&A, 365, 476

Oudmaijer, R. D., Waters, L. B. F. M., van der Veen, W. E. C. J., \& Geballe, T. R. 1995, A\&A, 299, 69

Pereira, C. B., \& Miranda, L. F. 2007, A\&A, 462, 231

Pottasch, S. R., Olling, R., Bignell, C., \& Zijlstra, A. A. 1988, A\&A, 205, 248

Preite-Martinez, A. 1988, A\&AS, 76, 317

Price, S. D., Egan, M. P., Carey, S. J., Mizuno, D. R., \& Kuchar, T. A. 2001, AJ, 121,2819

Ratag, M. A., Pottasch, S. R., Zijlstra, A. A., \& Menzies, J. 1990, A\&A, 233, 181

Sahai, R., Morris, M., Sánchez Contreras, C., \& Claussen, M. 2007, AJ, 134, 2200

Sevenster, M. N. 2002, AJ, 123, 2772

Siódmiak, N., Meixner, M., Ueta, T., et al. 2008, ApJ, 677, 382

Sivagnaman, P. 1989, Ph.D. Thesis, University of Paris VII, France

Skrutskie, M. F., Cutri, R. M., Stiening, R., et al. 2006, AJ, 131, 1163

Suárez, O., García-Lario, P., Manchado, A., et al. 2006, A\&A, 458, 173

te Lintel Hekkert, P., Caswell, J. L., Haynes, R. F., Norris, R. P., \& Habing, H. J. 1991, A\&AS, 90, 327

van de Steene, G. C. M., \& Pottasch, S. R. 1993, A\&A, 274, 895

van de Steene, G. C. M., van Hoof, P. A. M., \& Wood, P. R. 2000, A\&A, 362, 984

van der Veen, W. E. C. J., \& Habing, H. J. 1988, A\&A, 194, 125

van der Veen, W. E. C. J., Habing, H. J., \& Geballe, T. R. 1989, A\&A, 226, 108 

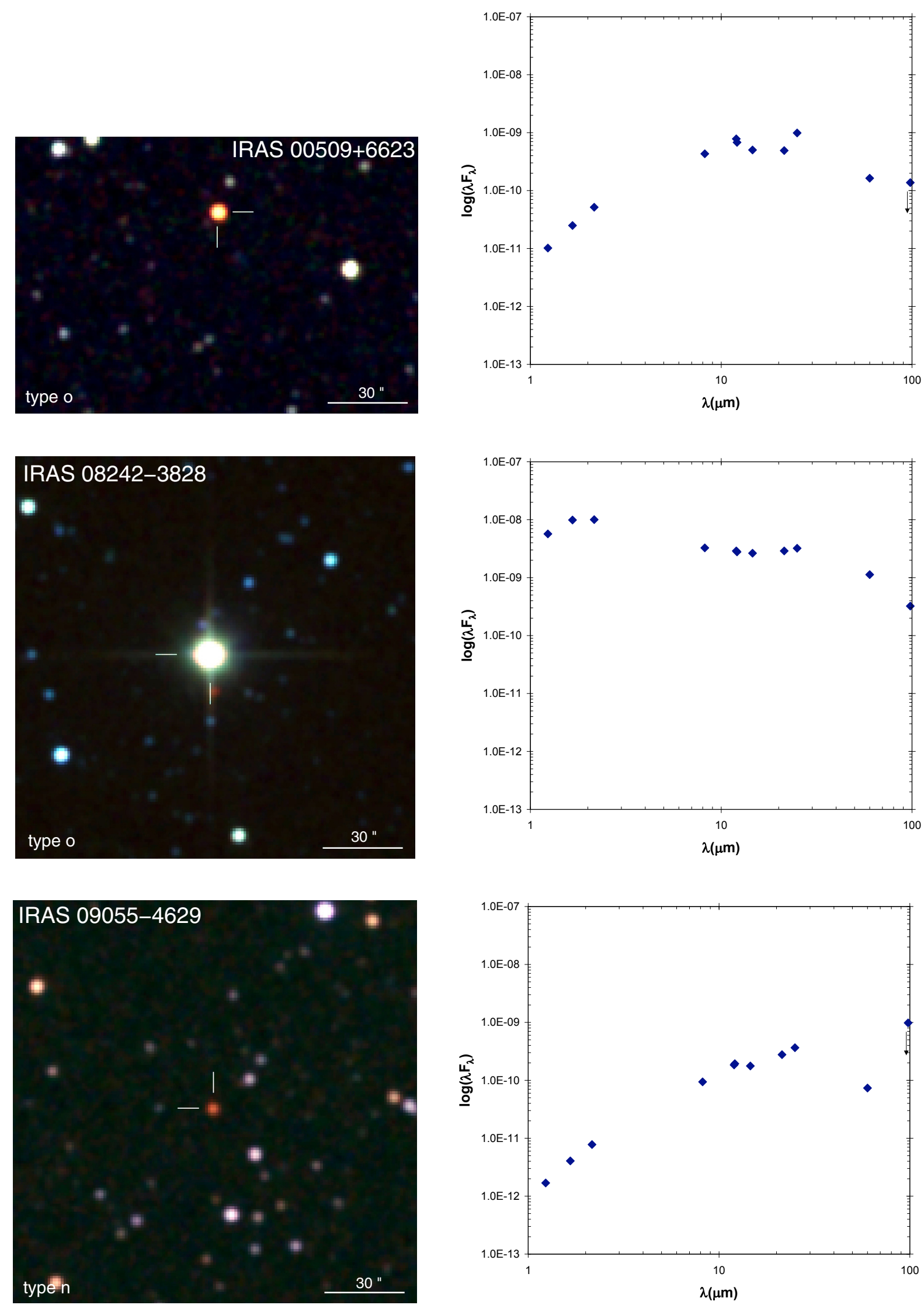

Fig. 3. (left) 2MASS $J$ (blue), $H$ (green), and $K_{\mathrm{S}}$ (red) composite pictures and (right) IR spectral energy distributions (SEDs) of the IRAS postAGB star and PN candidates with near-IR counterparts in the 2MASS PSC. In the pictures, north is up, east to the left, and the locations of the near-IR counterparts and their types ( $o$ or $n$ whether they are detected in DSS red images or not) are overlaid. The SEDs are built using data extracted from the 2MASS, Spitzer GLIMPSE, MSX, and IRAS catalogs. The arrows at $100 \mu \mathrm{m}$ represent flux upper limits (IRAS quality-factor, FQUAL, equals to 1). 
G. Ramos-Larios et al.: IRAS obscured post-AGB star and PN candidates with 2MASS counterparts. I.
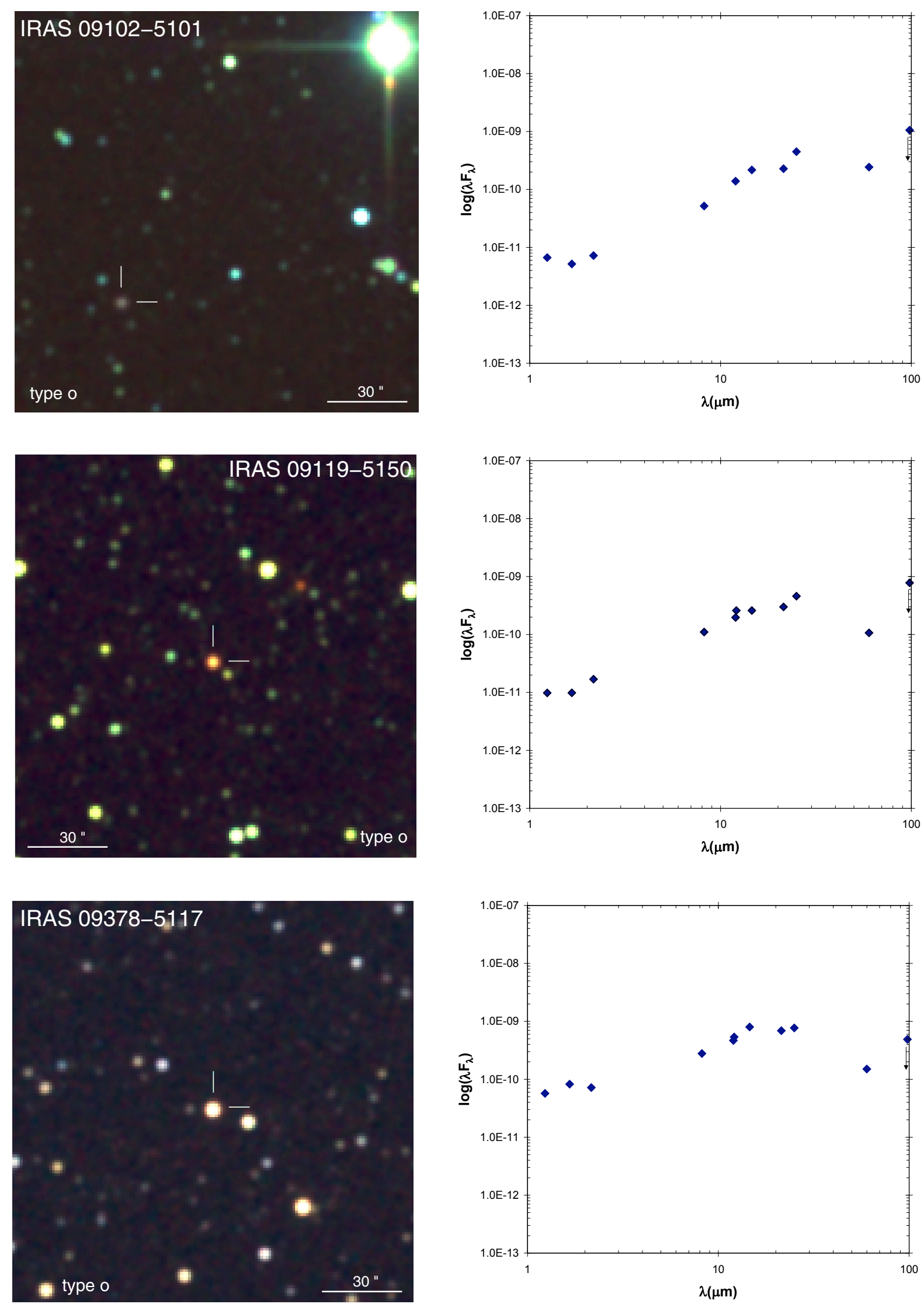

Fig. 3. continued. 

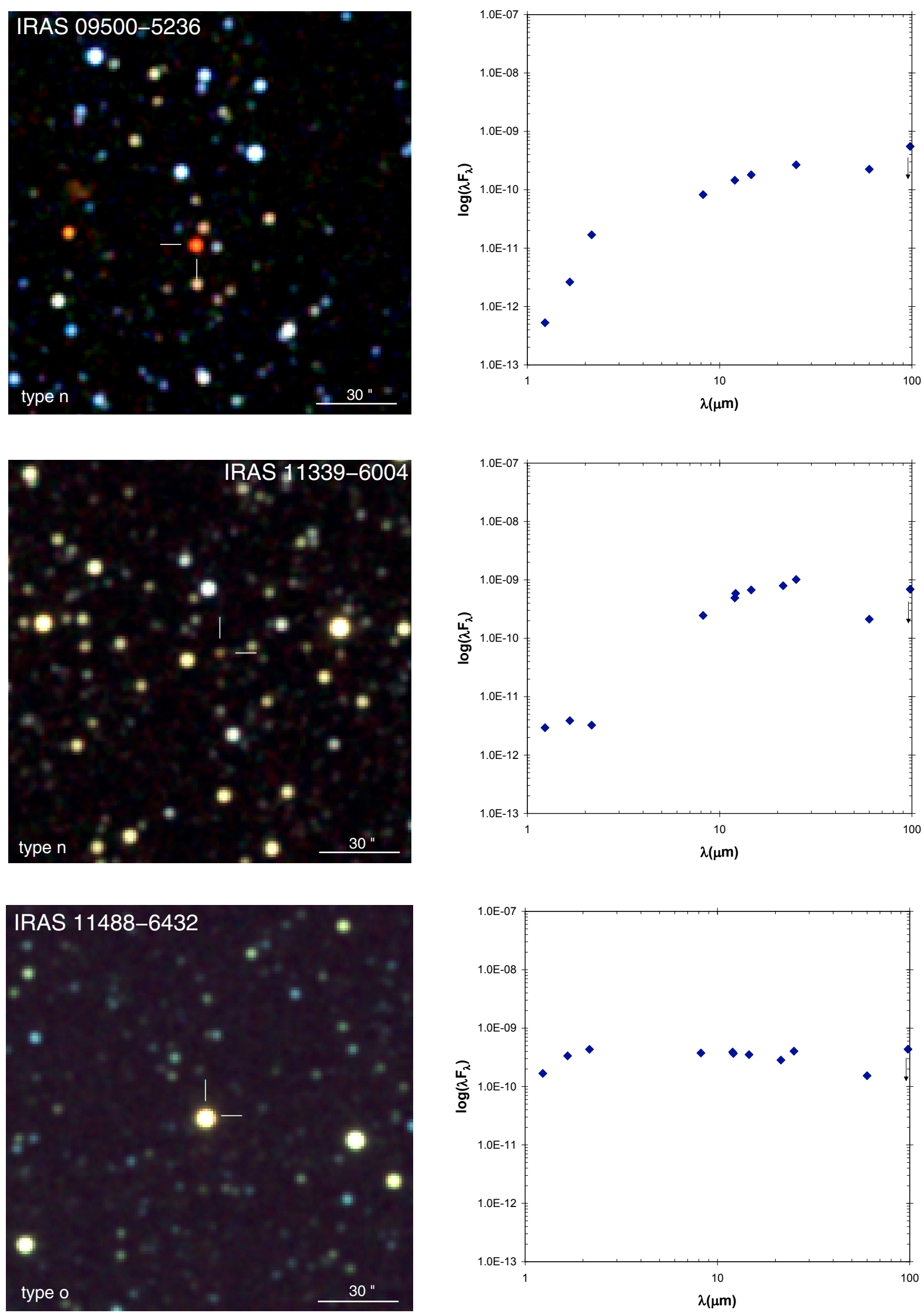

Fig. 3. continued. 
G. Ramos-Larios et al.: IRAS obscured post-AGB star and PN candidates with 2MASS counterparts. I.
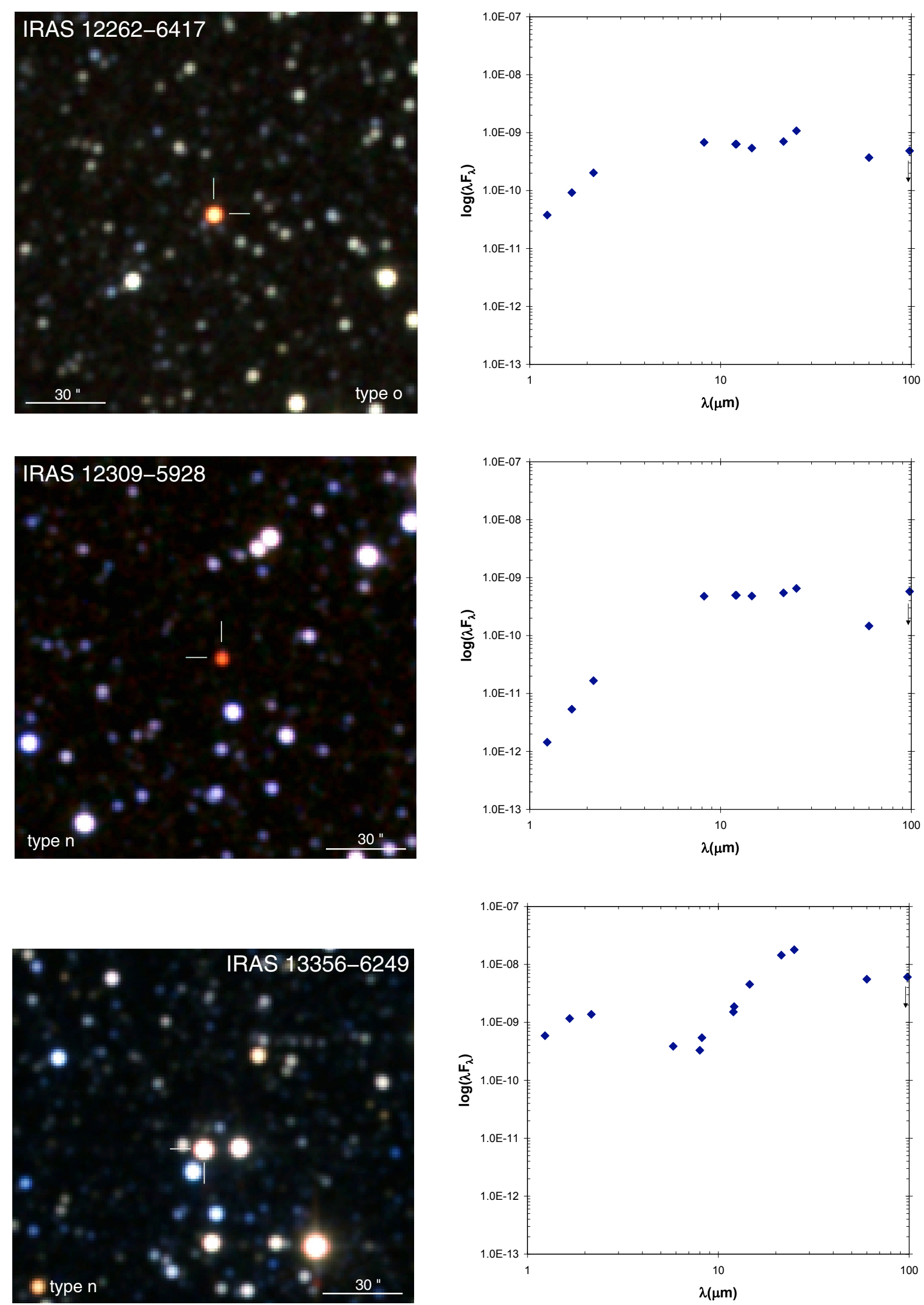

Fig. 3. continued. 

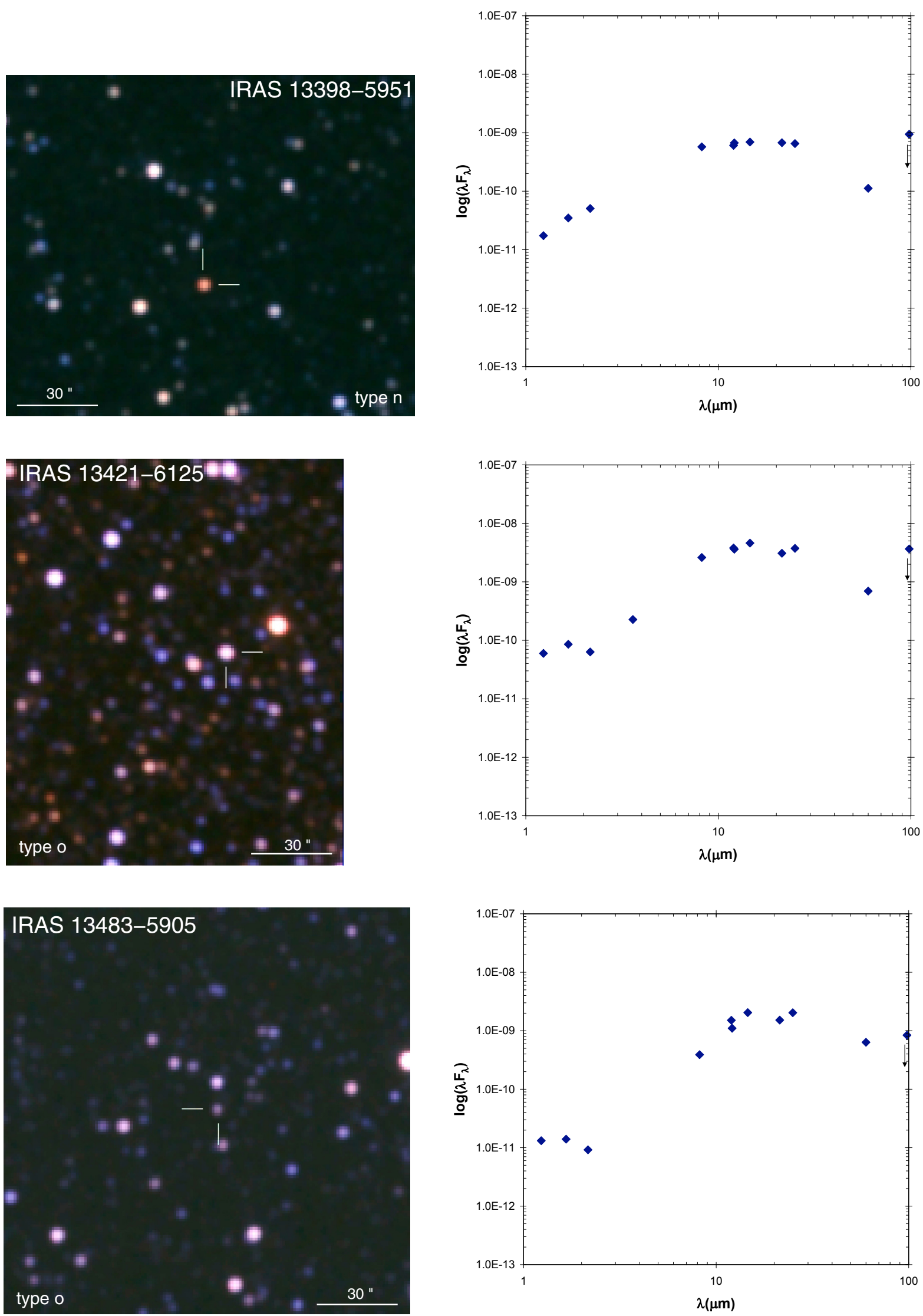

Fig. 3. continued. 
G. Ramos-Larios et al.: IRAS obscured post-AGB star and PN candidates with 2MASS counterparts. I.
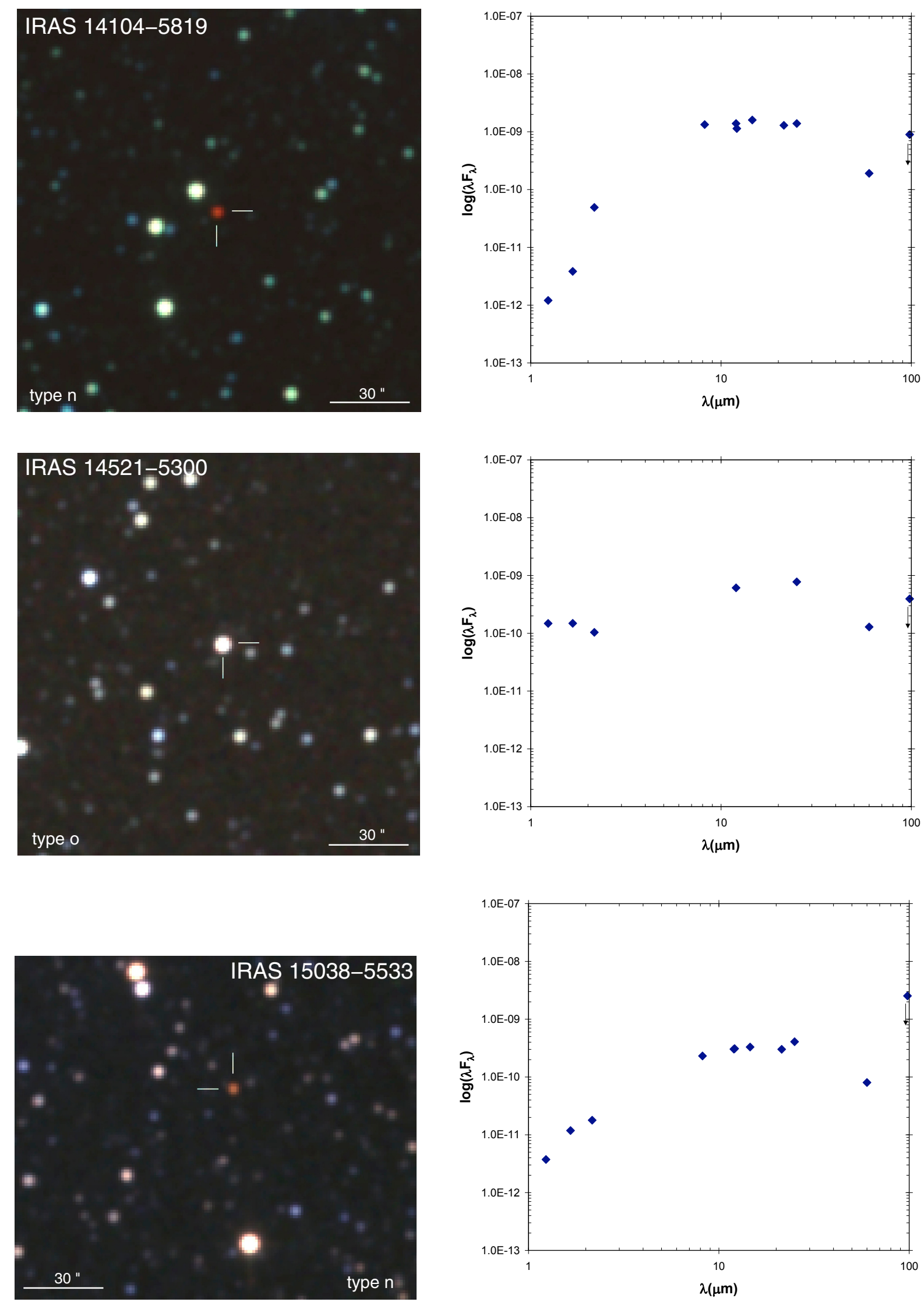

Fig. 3. continued. 

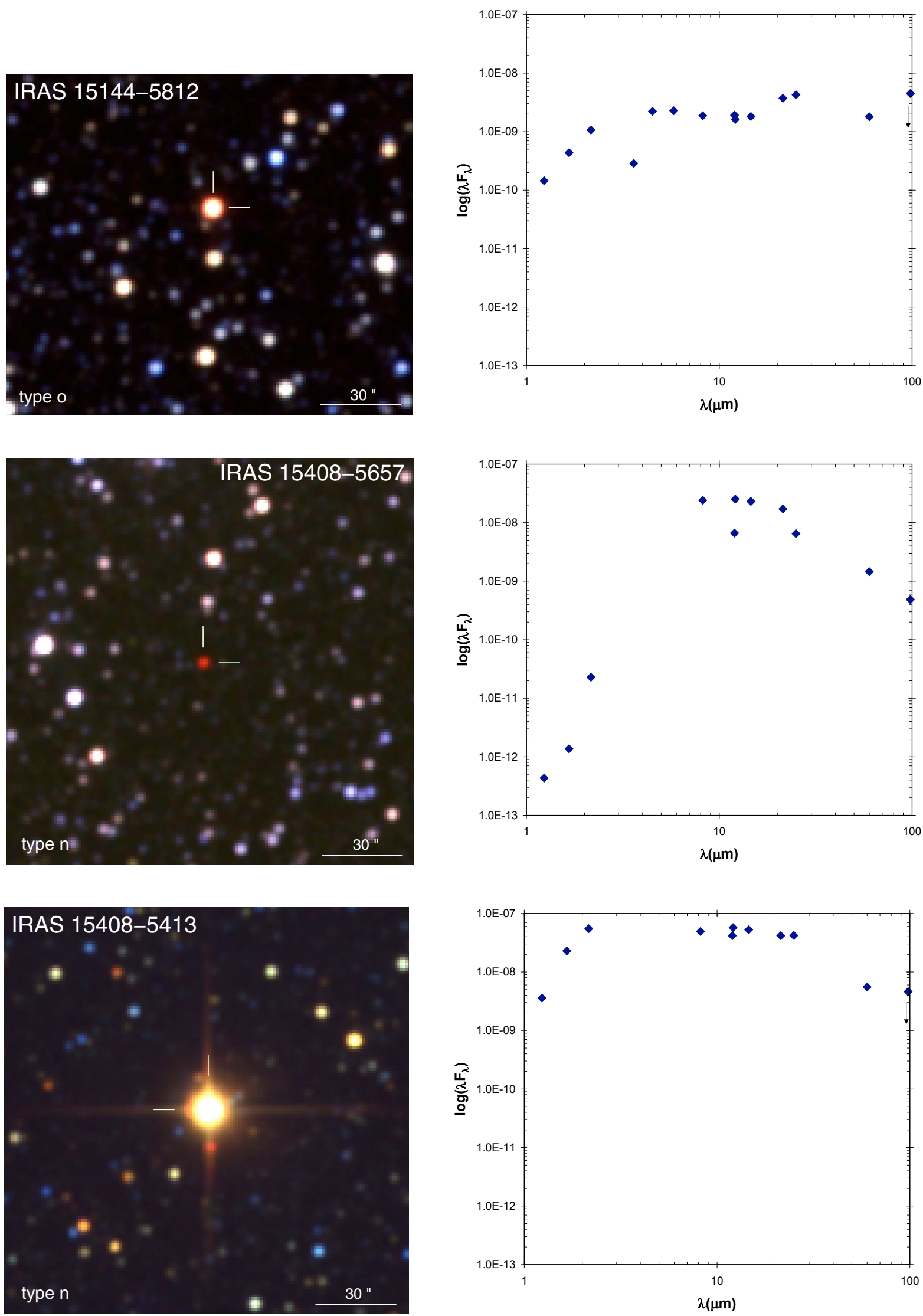

Fig. 3. continued. 
G. Ramos-Larios et al.: IRAS obscured post-AGB star and PN candidates with 2MASS counterparts. I.
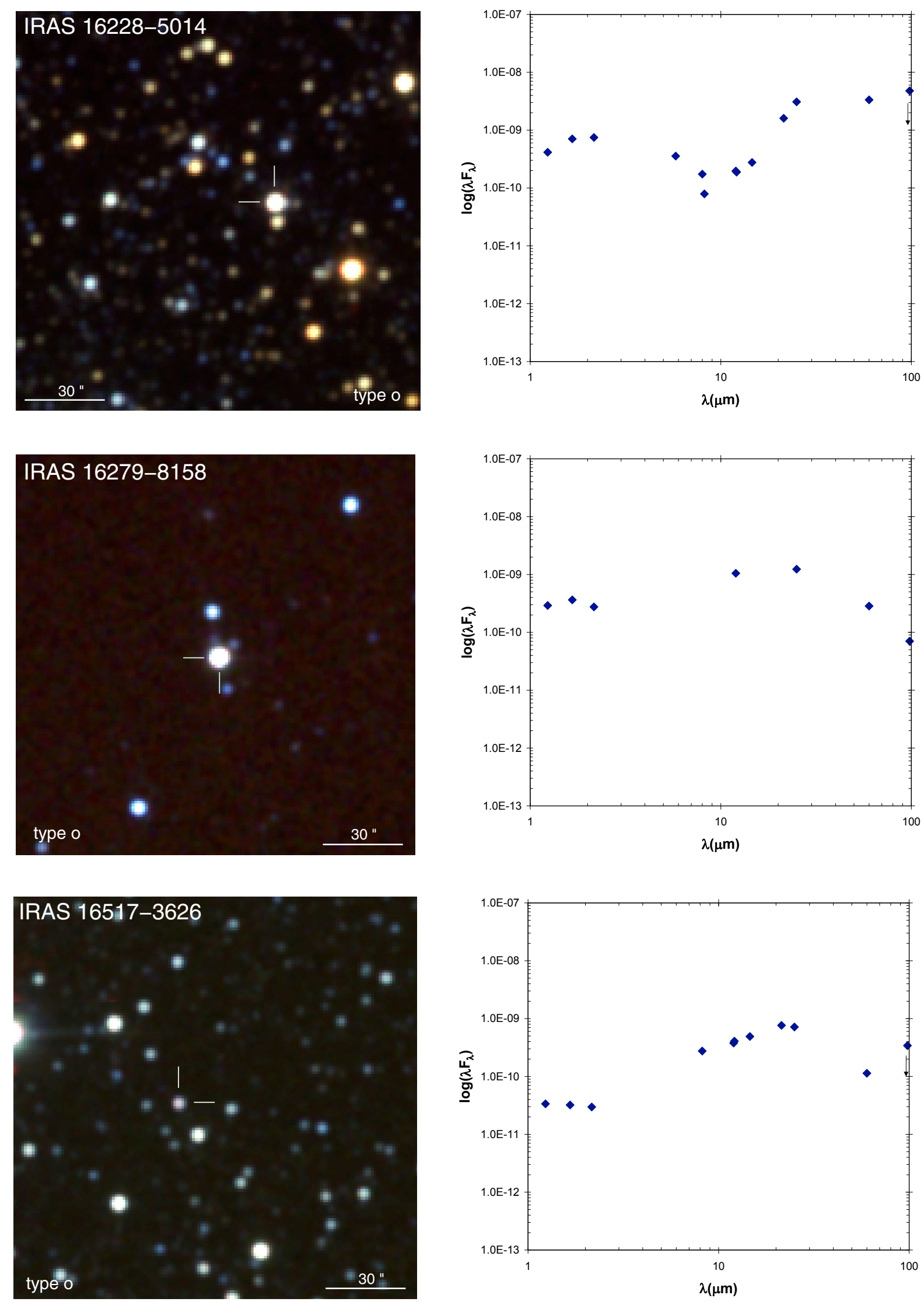

Fig. 3. continued. 

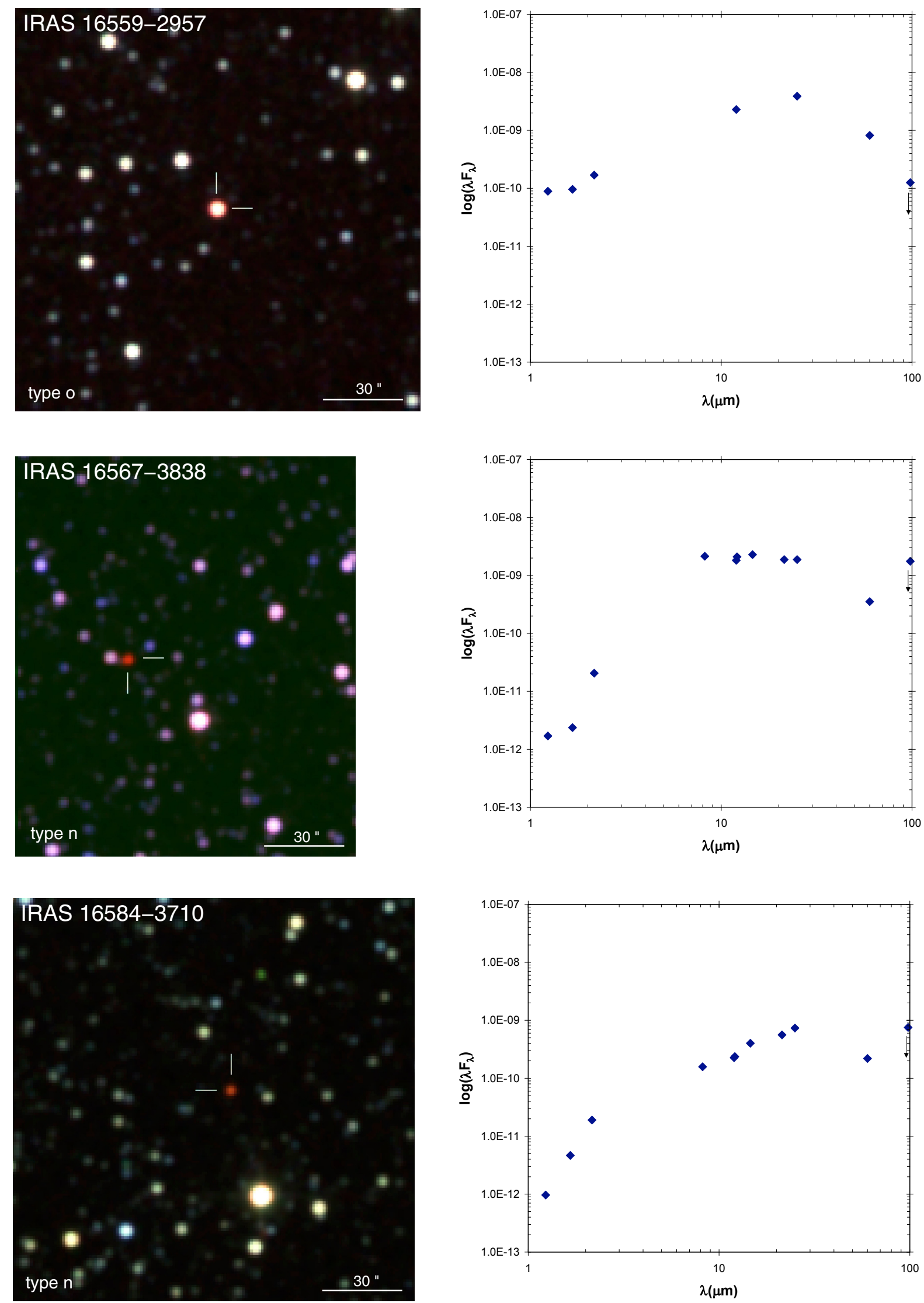

Fig. 3. continued. 
G. Ramos-Larios et al.: IRAS obscured post-AGB star and PN candidates with 2MASS counterparts. I.
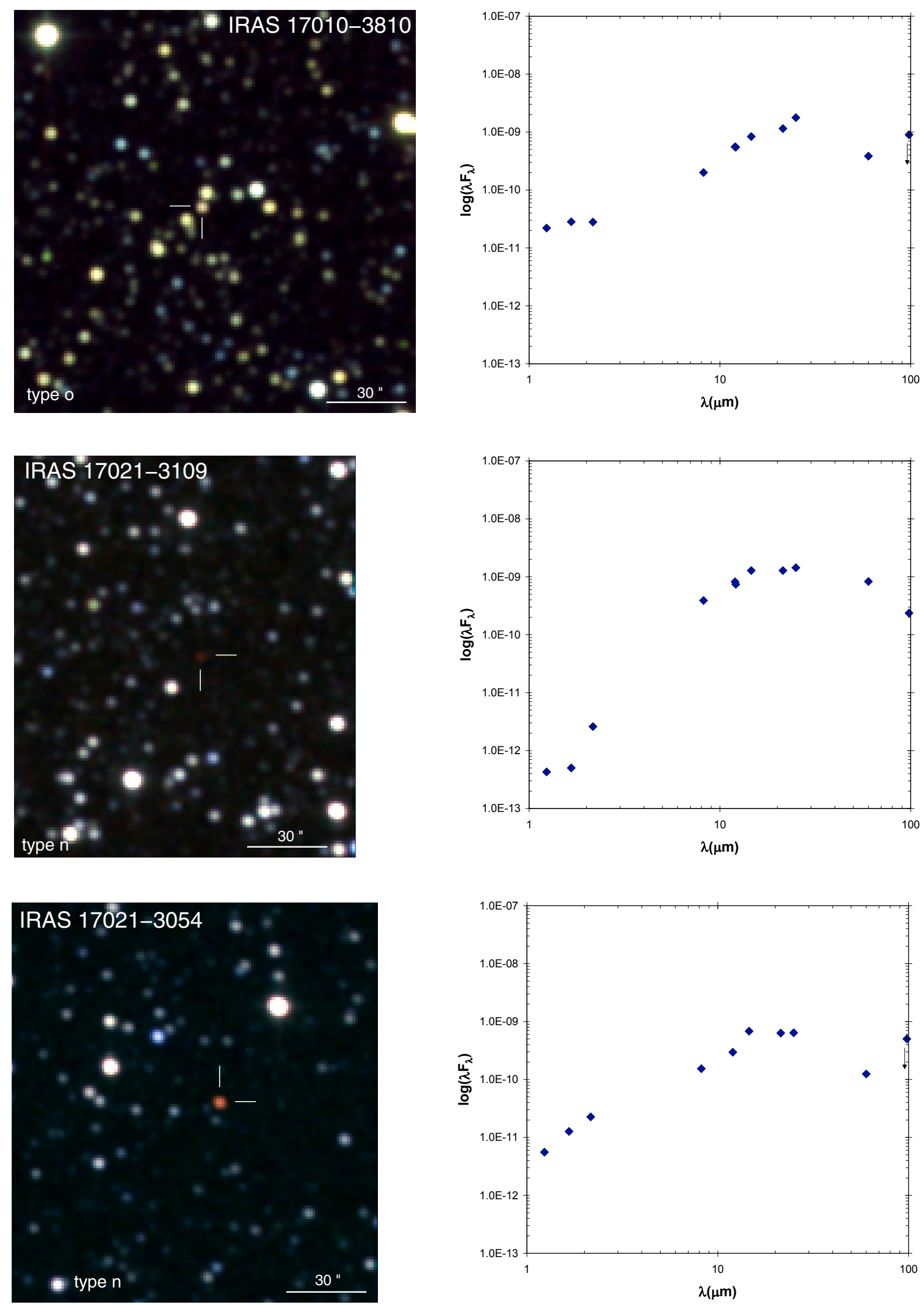

Fig. 3. continued. 

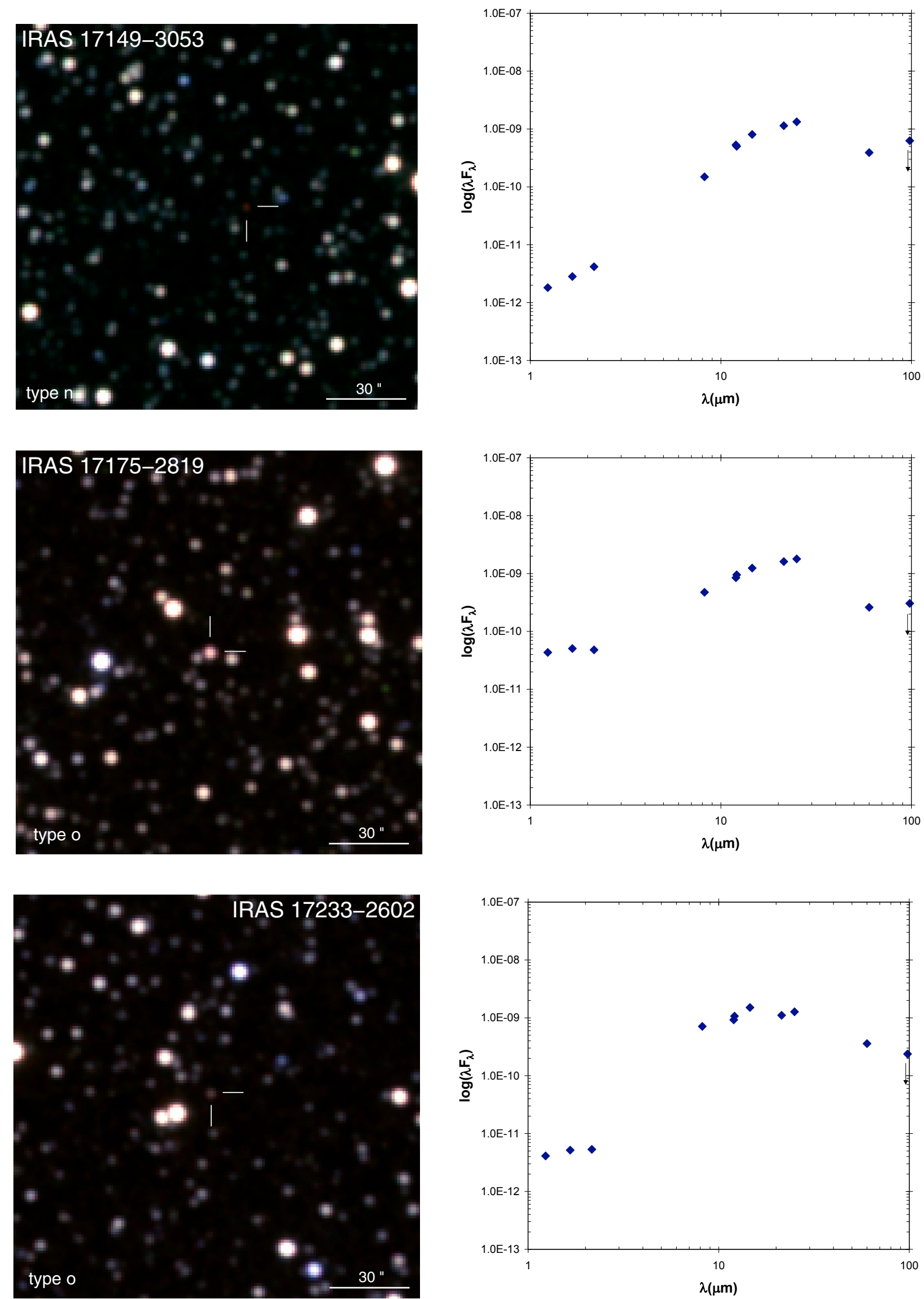

Fig. 3. continued. 
G. Ramos-Larios et al.: IRAS obscured post-AGB star and PN candidates with 2MASS counterparts. I.
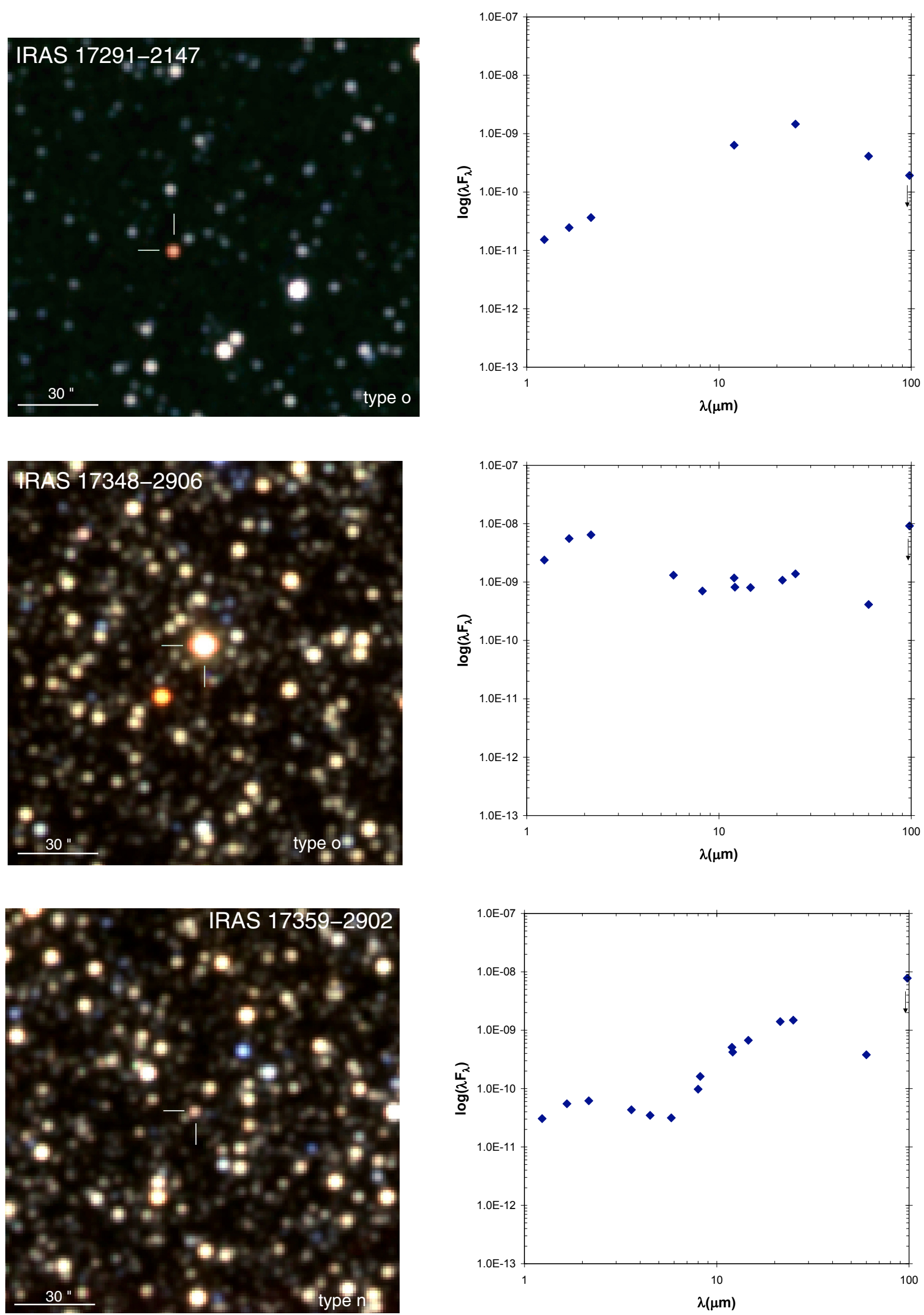

Fig. 3. continued. 

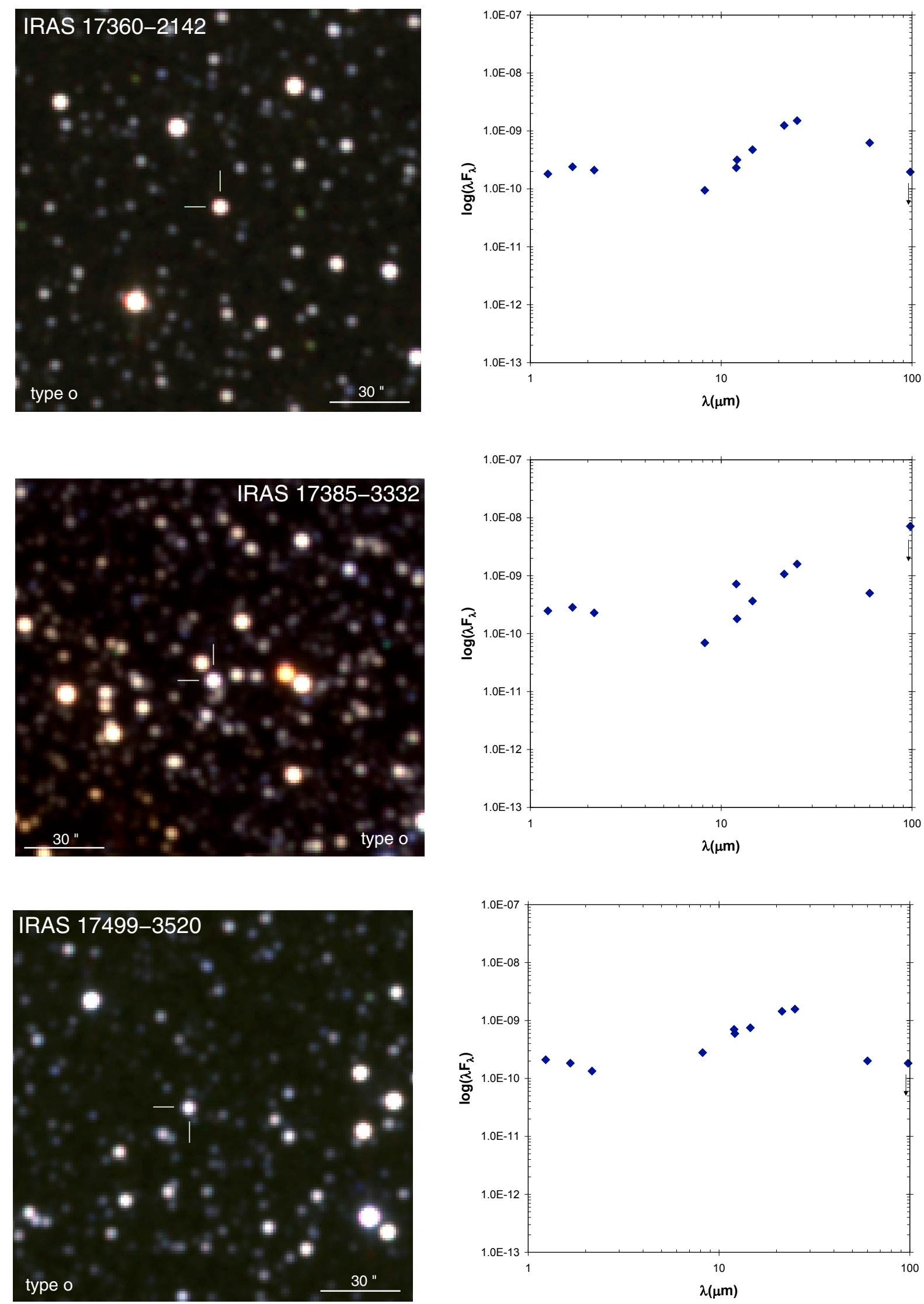

Fig. 3. continued. 
G. Ramos-Larios et al.: IRAS obscured post-AGB star and PN candidates with 2MASS counterparts. I.
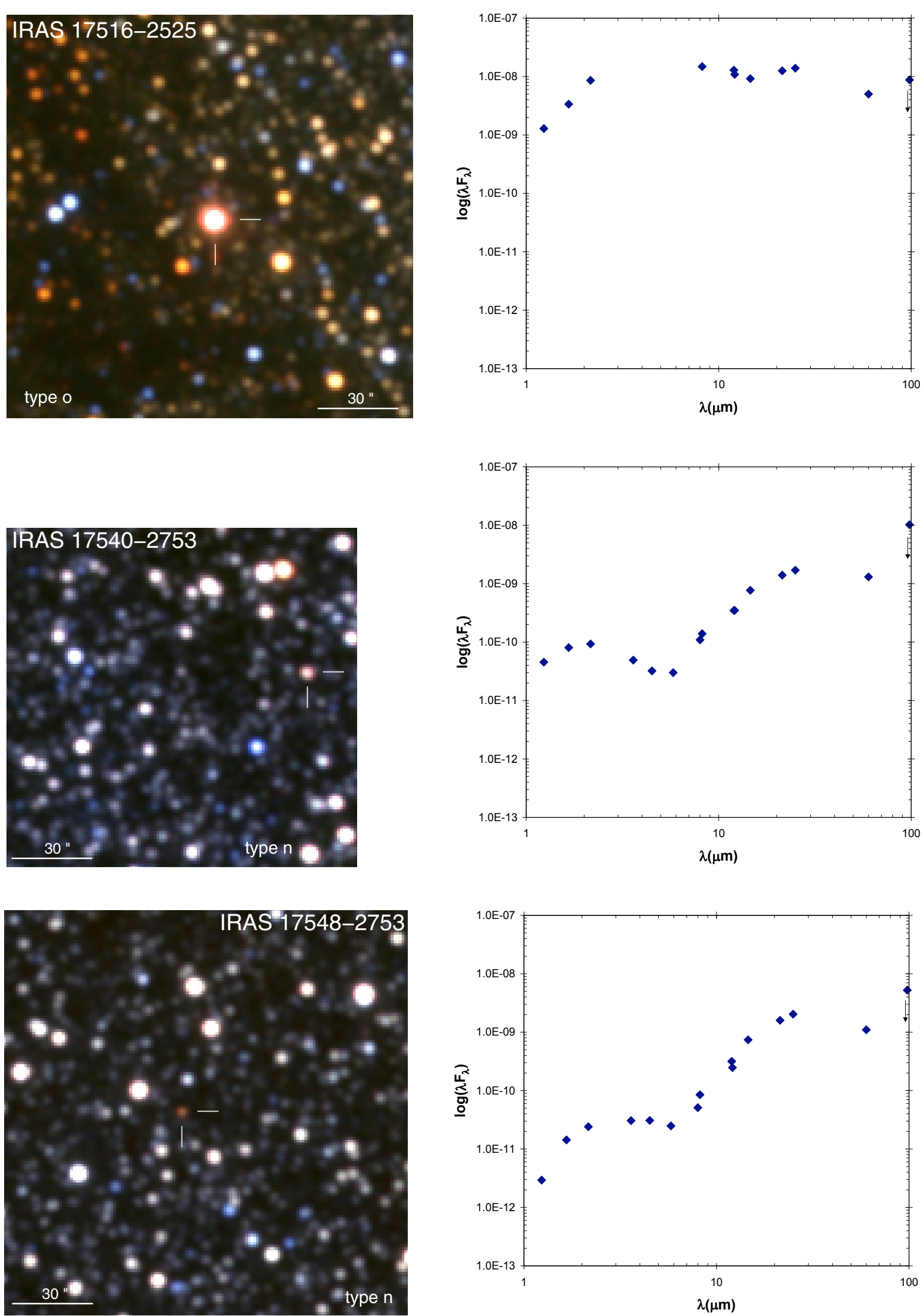

Fig. 3. continued. 

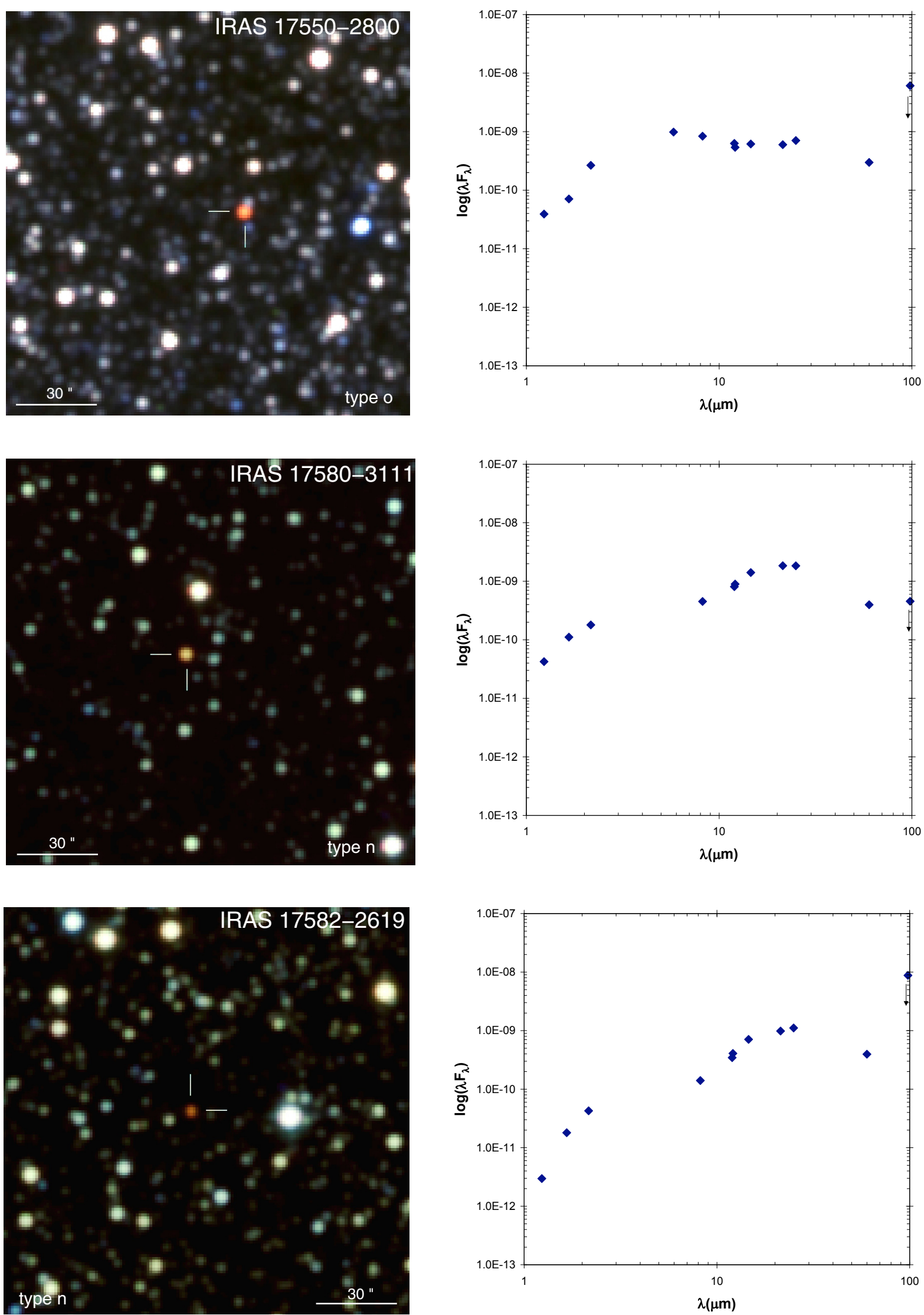

Fig. 3. continued. 
G. Ramos-Larios et al.: IRAS obscured post-AGB star and PN candidates with 2MASS counterparts. I.
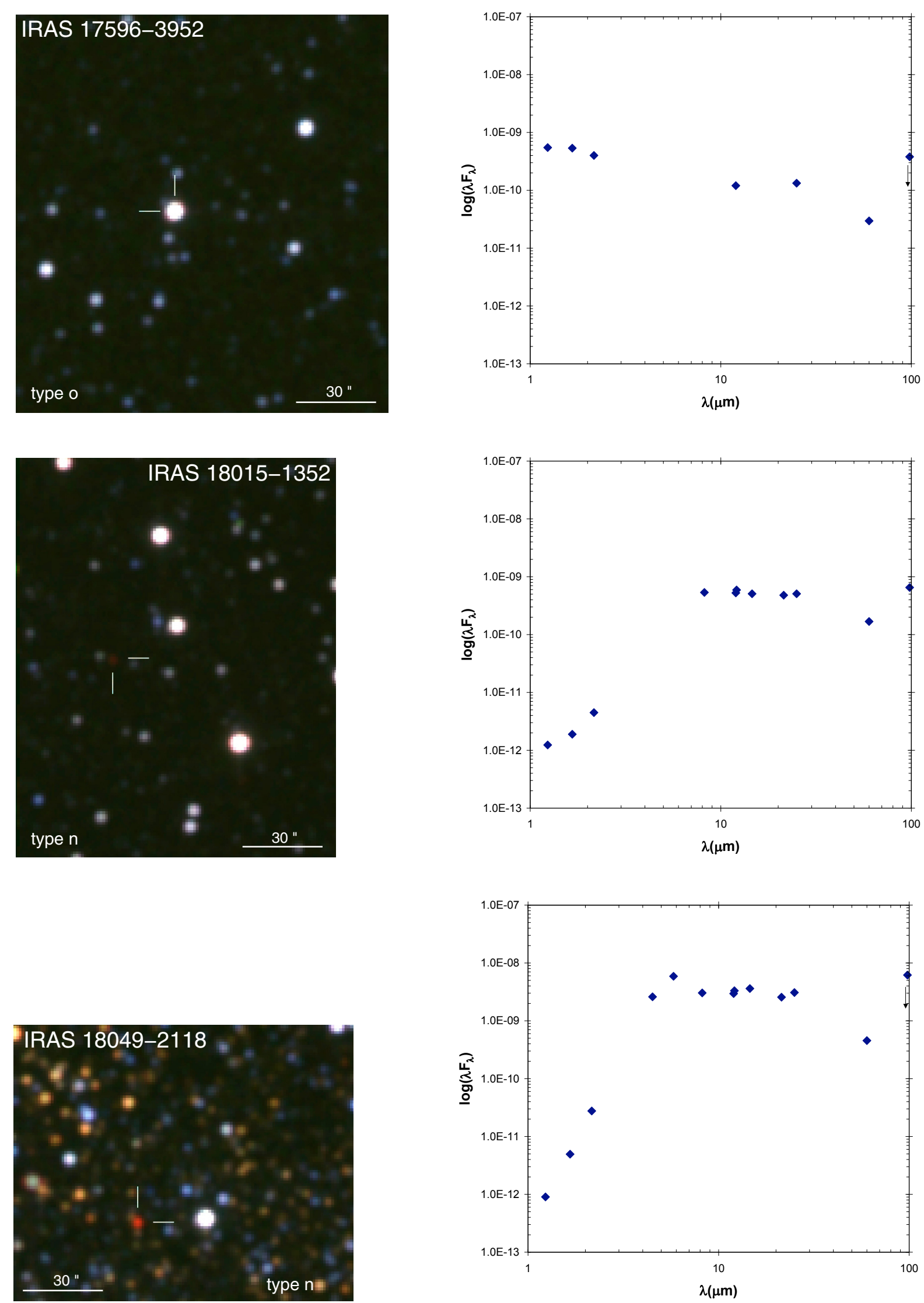

Fig. 3. continued. 

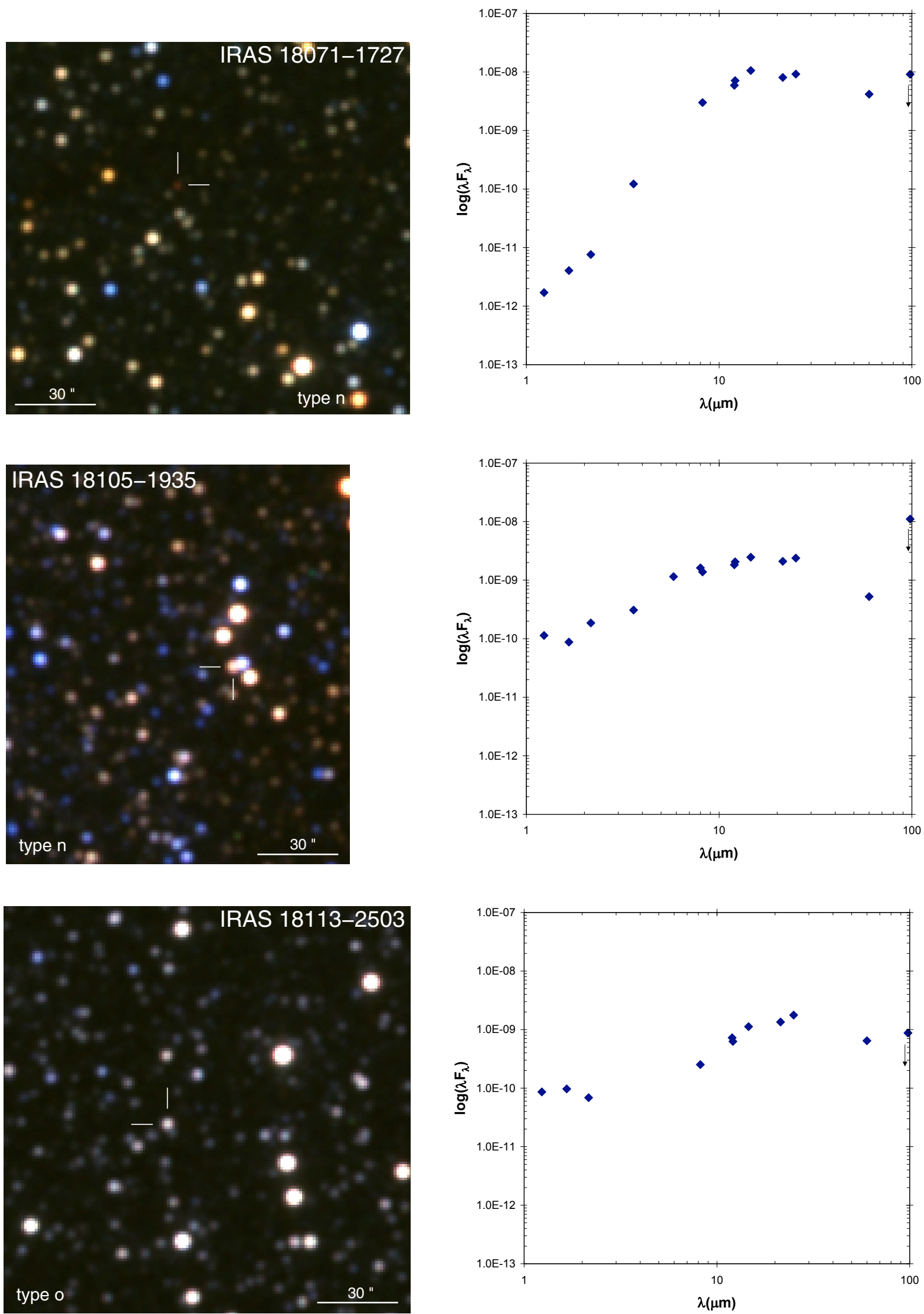

Fig. 3. continued. 
G. Ramos-Larios et al.: IRAS obscured post-AGB star and PN candidates with 2MASS counterparts. I.
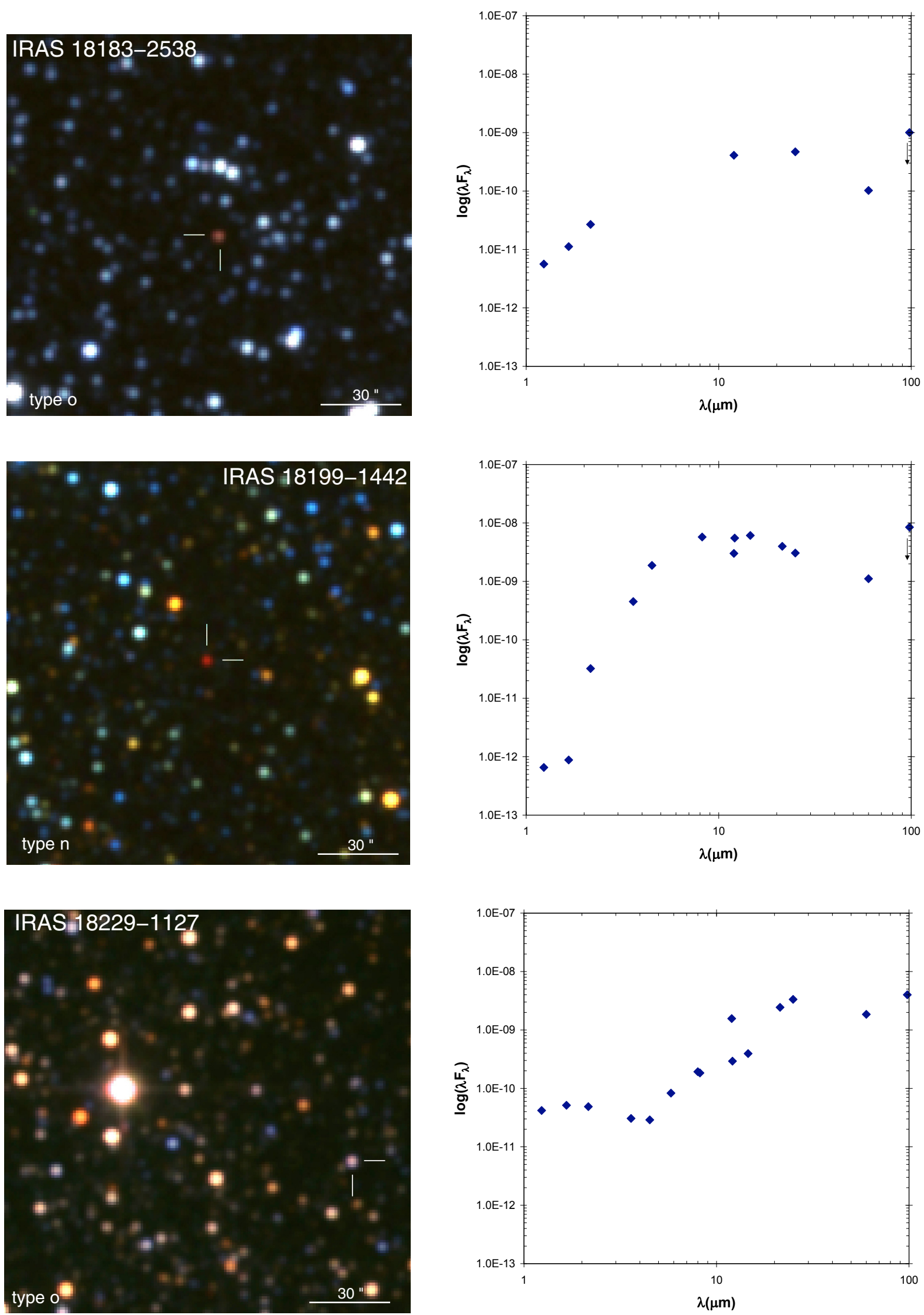

Fig. 3. continued. 

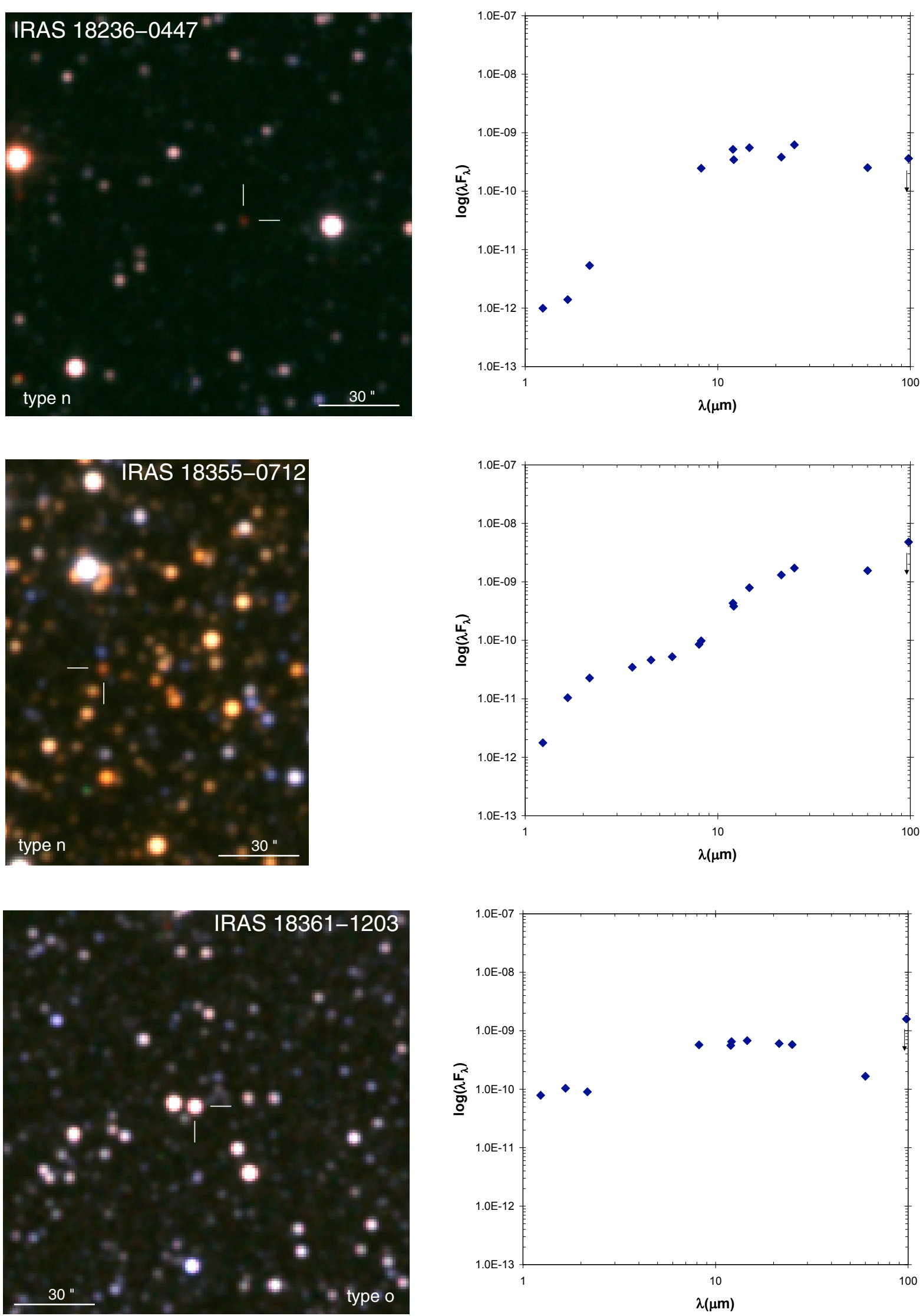

Fig. 3. continued. 
G. Ramos-Larios et al.: IRAS obscured post-AGB star and PN candidates with 2MASS counterparts. I.
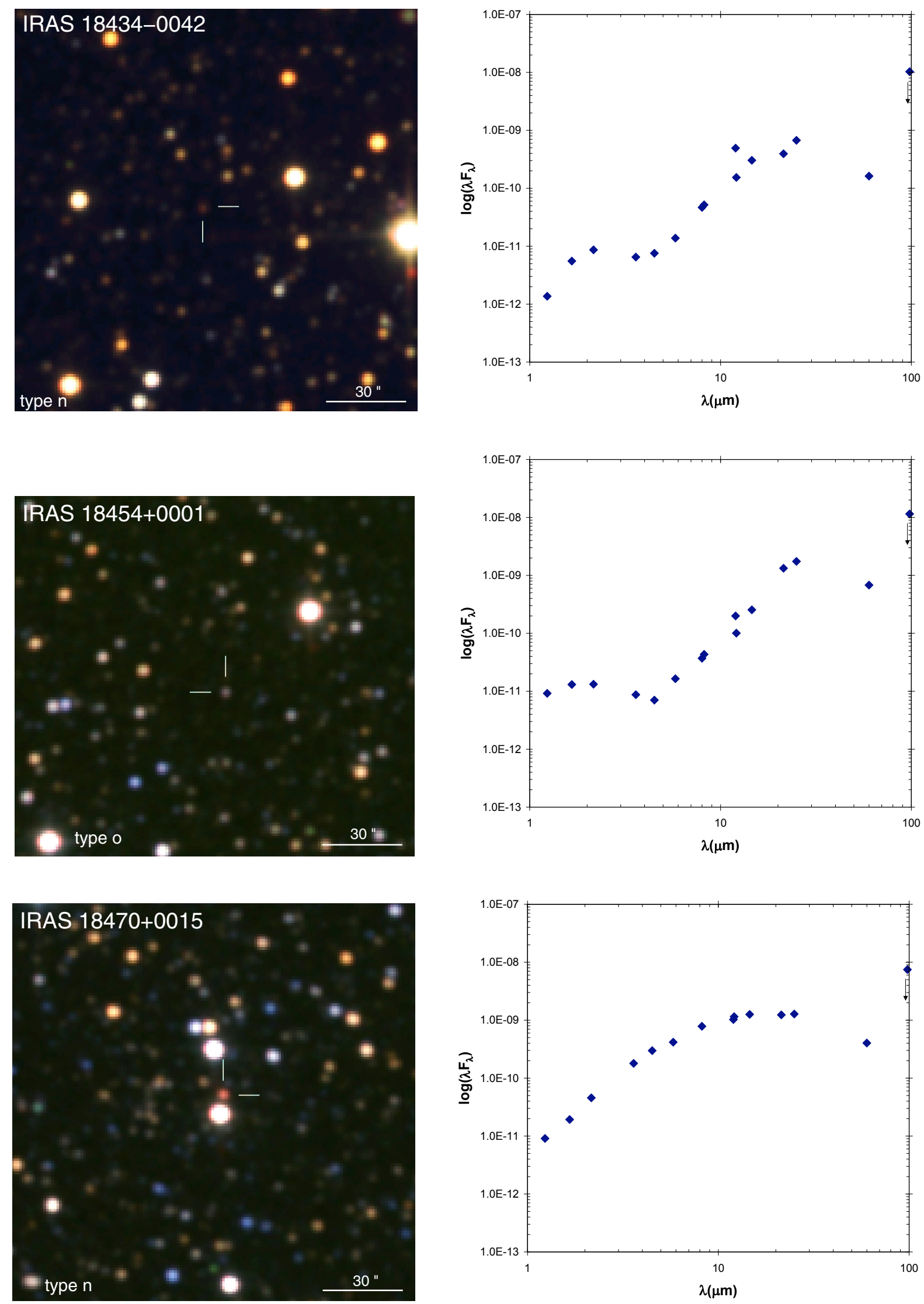

Fig. 3. continued. 

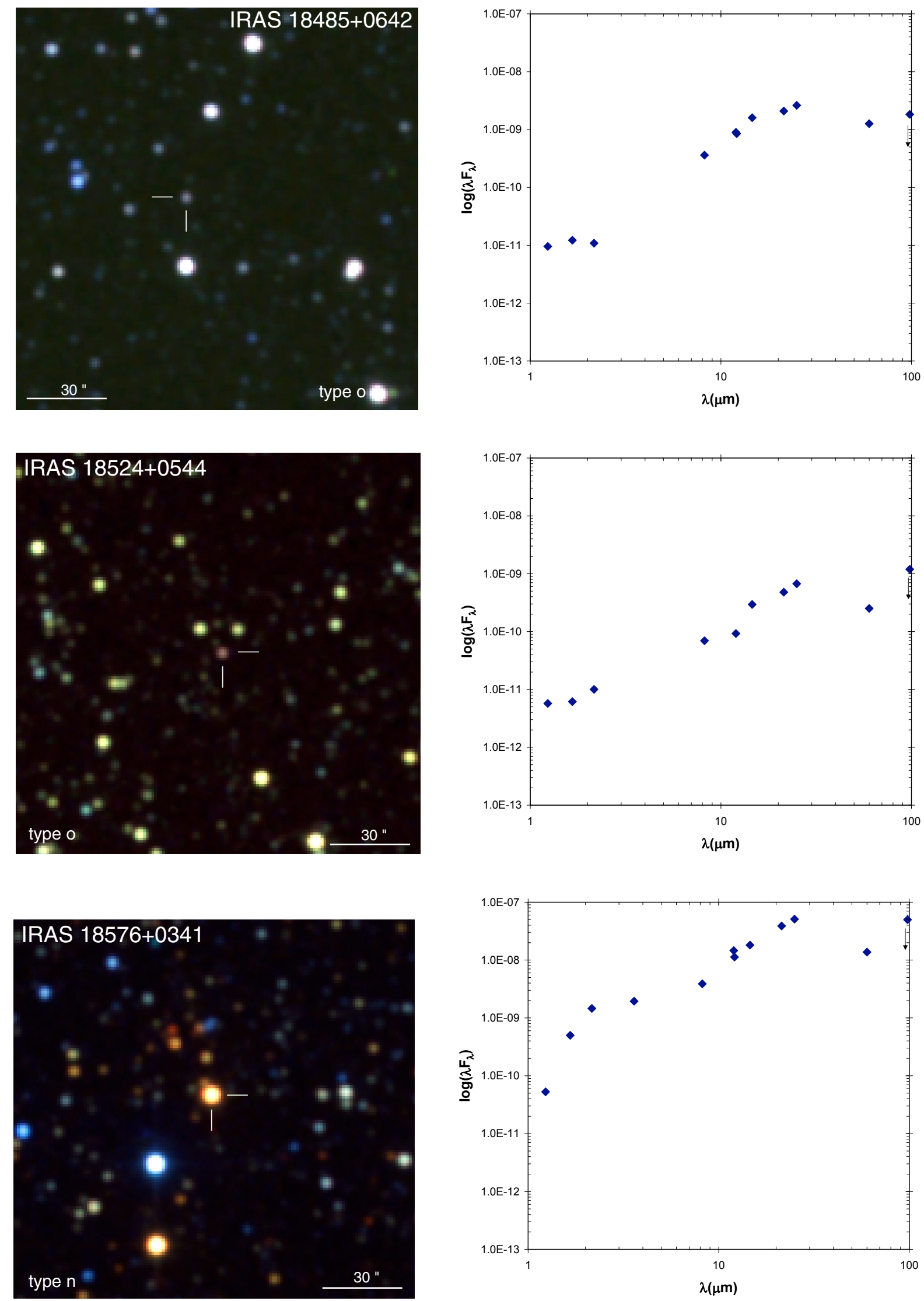

Fig. 3. continued. 
G. Ramos-Larios et al.: IRAS obscured post-AGB star and PN candidates with 2MASS counterparts. I.
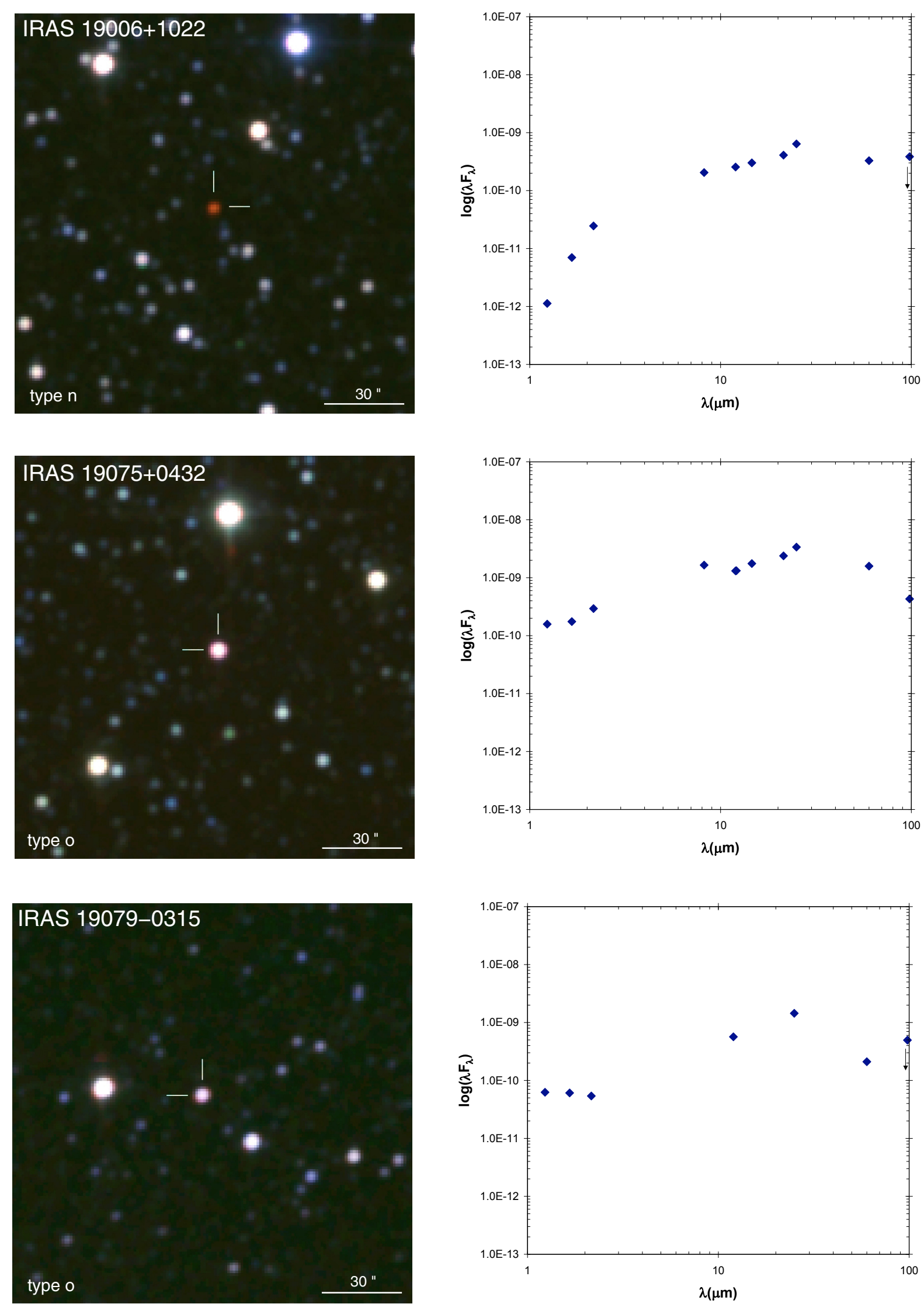

Fig. 3. continued. 

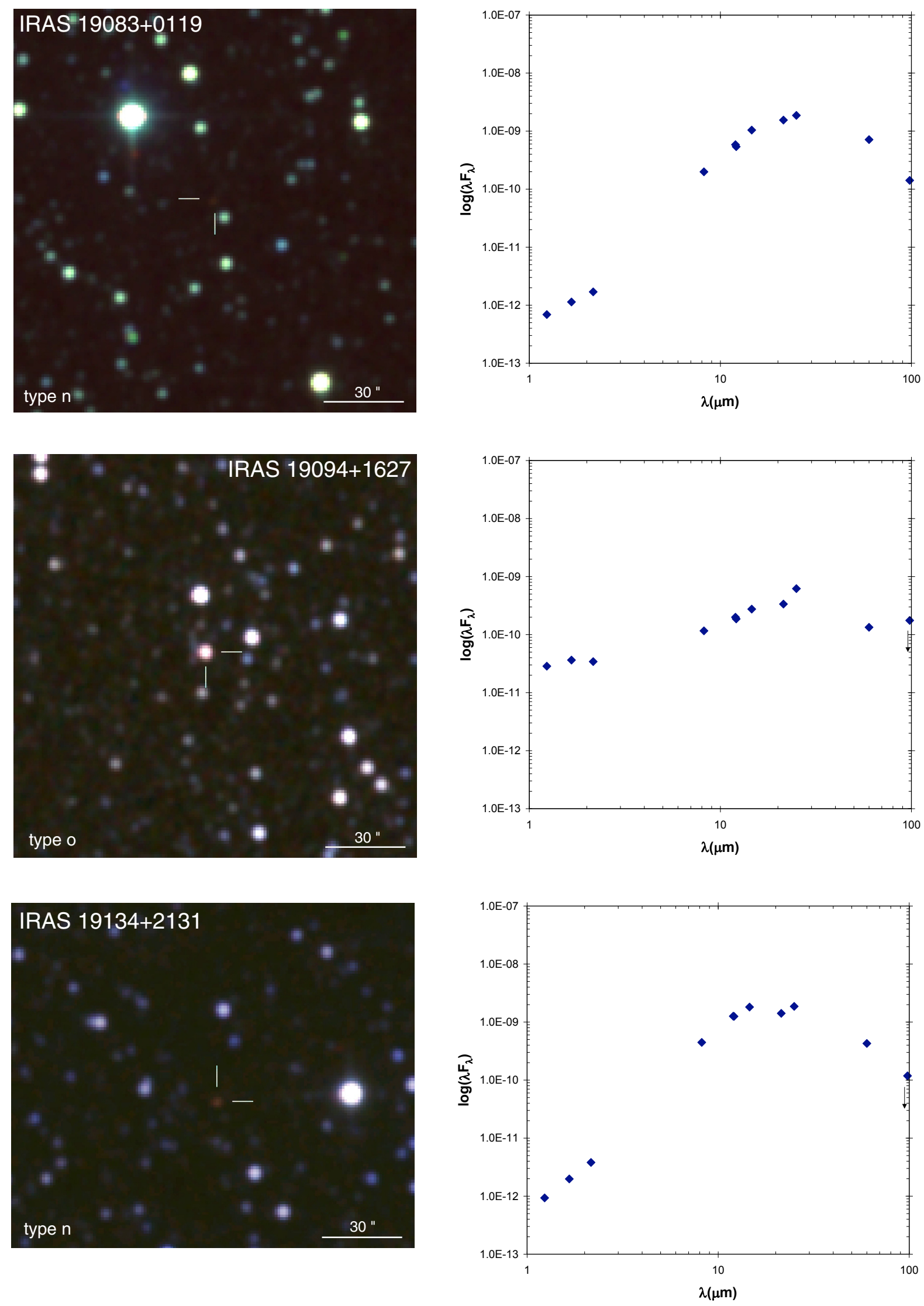

Fig. 3. continued. 
G. Ramos-Larios et al.: IRAS obscured post-AGB star and PN candidates with 2MASS counterparts. I.
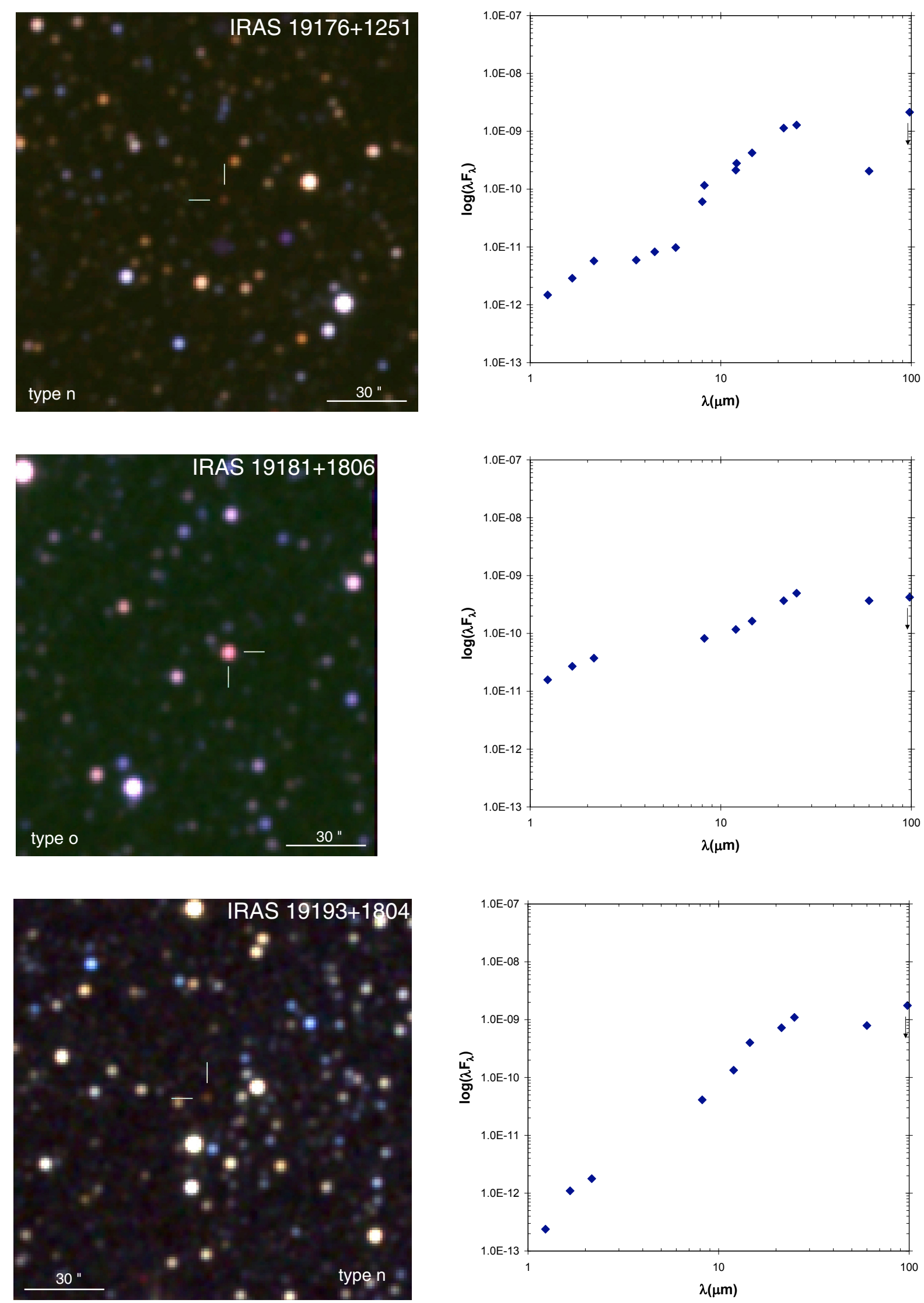

Fig. 3. continued. 

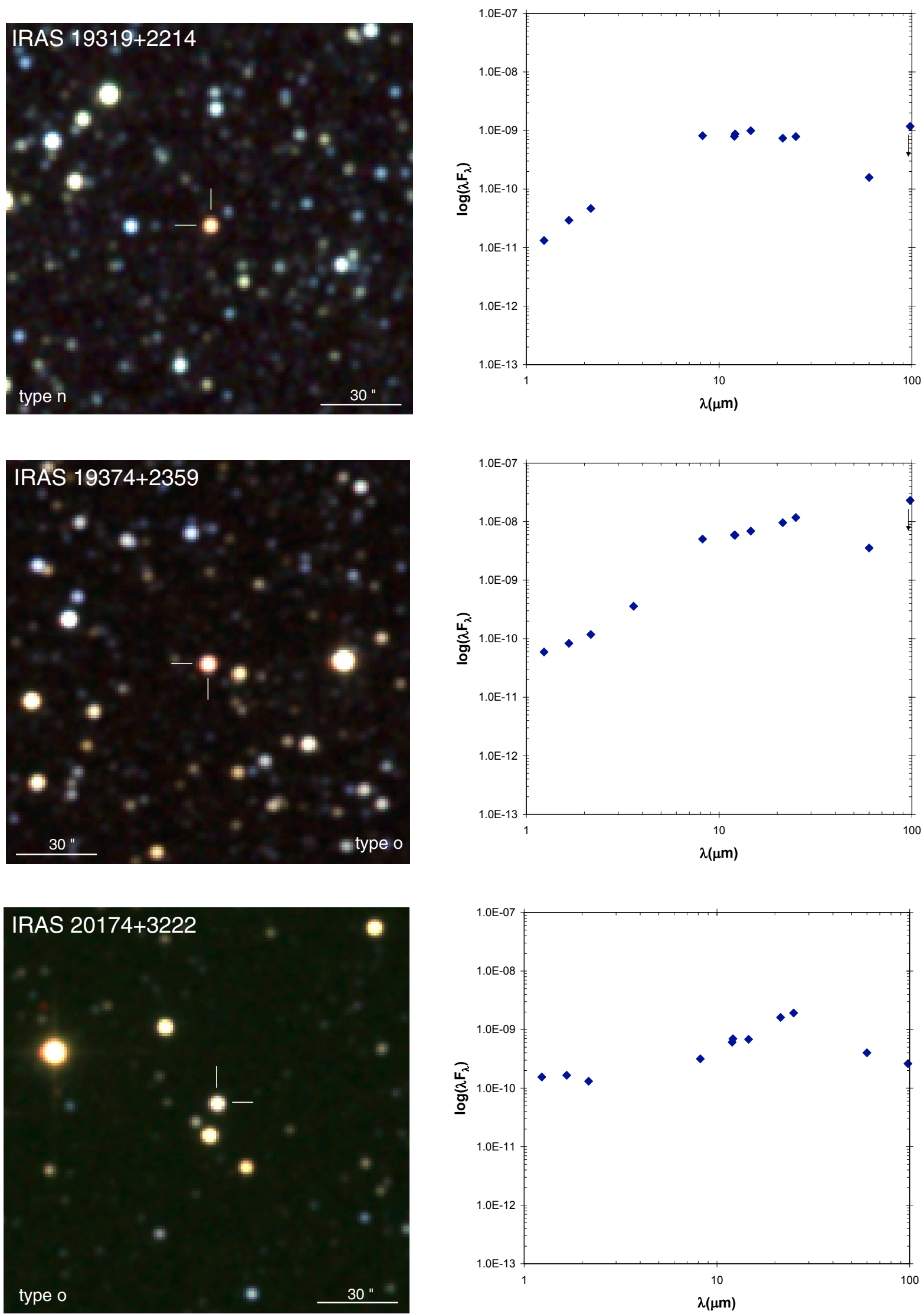

Fig. 3. continued. 
G. Ramos-Larios et al.: IRAS obscured post-AGB star and PN candidates with 2MASS counterparts. I.
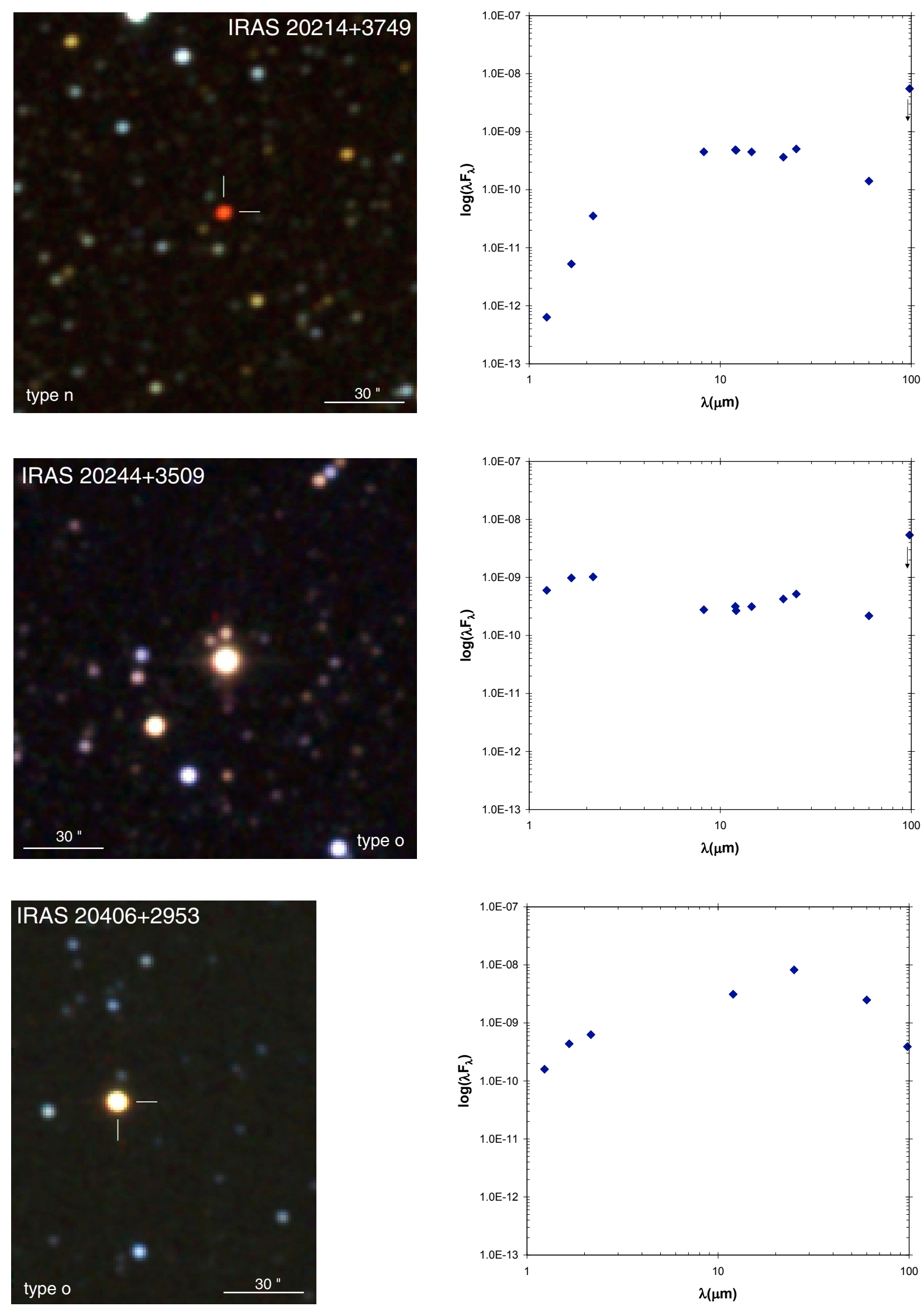

Fig. 3. continued. 

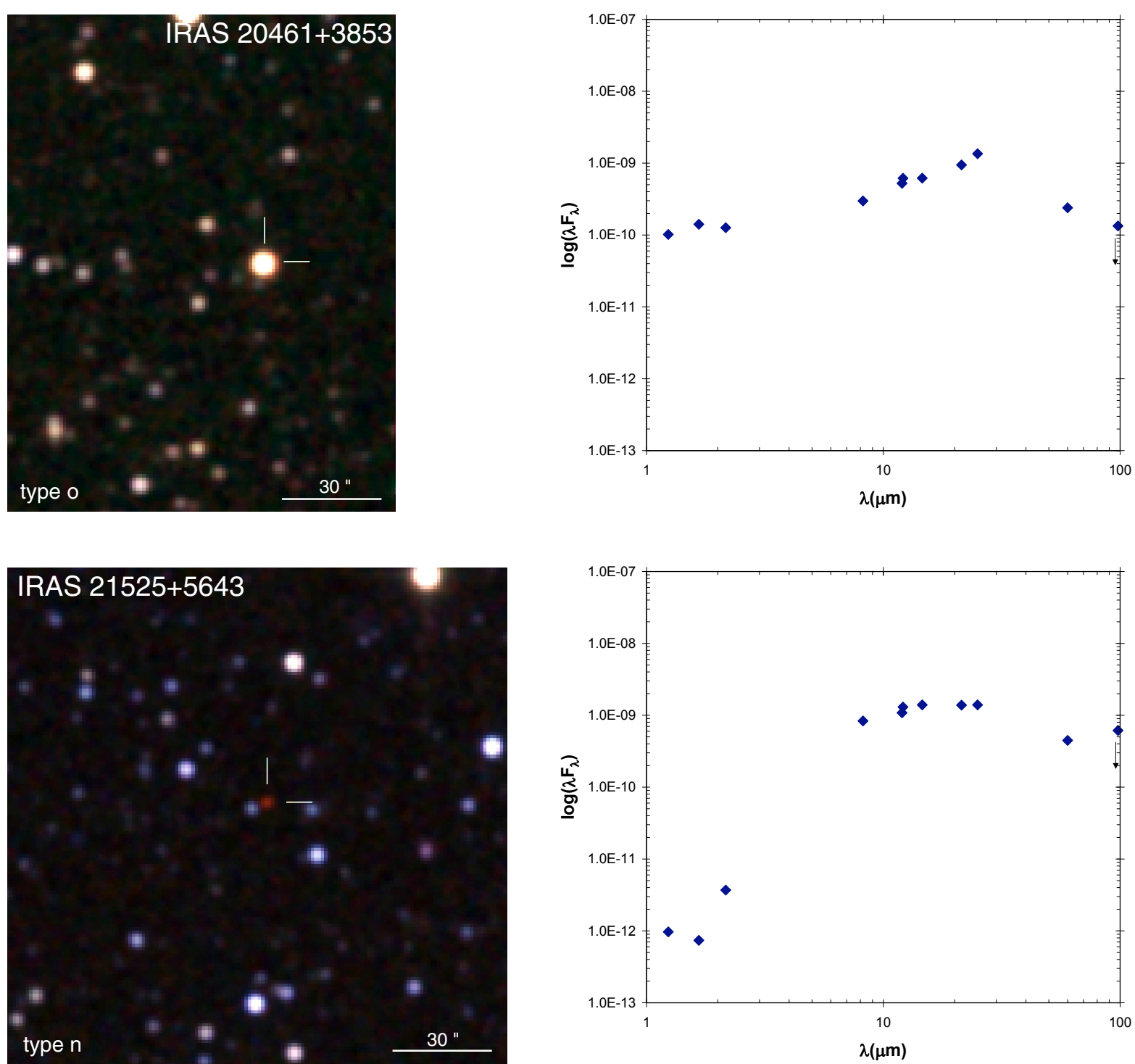

Fig. 3. continued. 

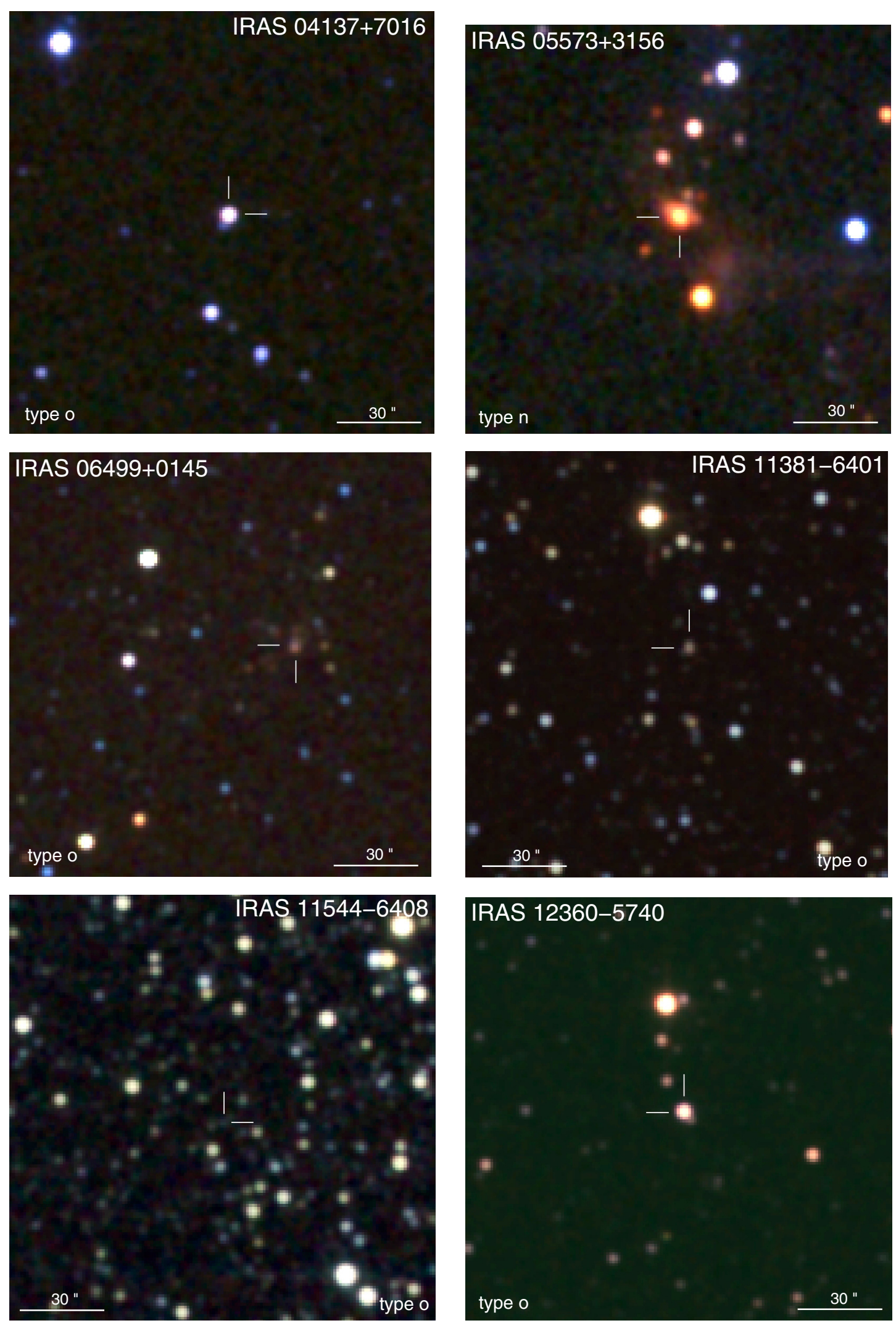

Fig. 4. 2MASS $J$ (blue), $H$ (green), and $K_{\mathrm{S}}$ (red) composite pictures of the IRAS post-AGB star and PN candidates with near-IR counterparts that are not resolved or unambiguously identified in the 2MASS PSC. The locations of the near-IR counterparts and their types ( $o$ or $n$ whether they are detected in DSS red images or not) are overlaid on the pictures. North is up, east to the left. 

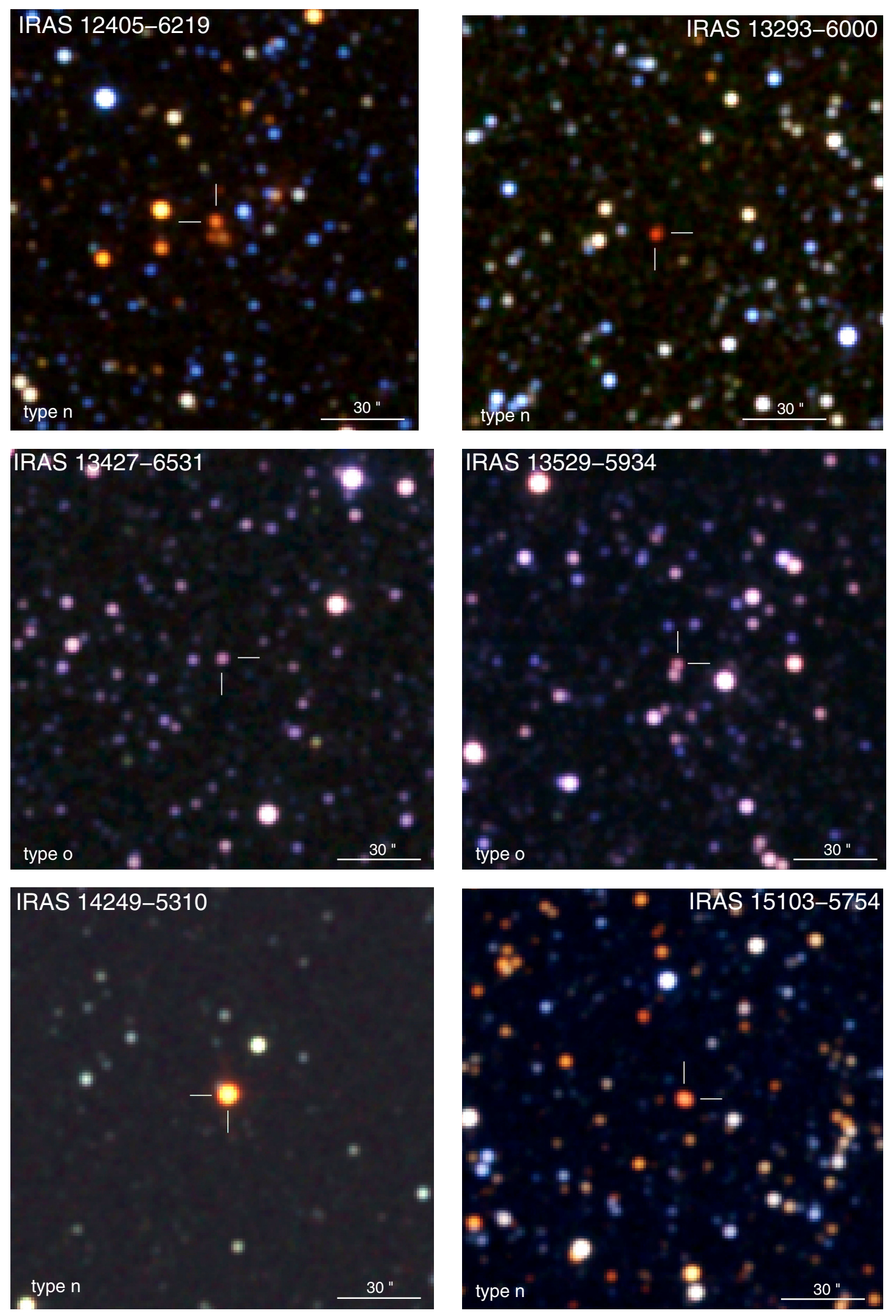

Fig. 4. continued. 
G. Ramos-Larios et al.: IRAS obscured post-AGB star and PN candidates with 2MASS counterparts. I.
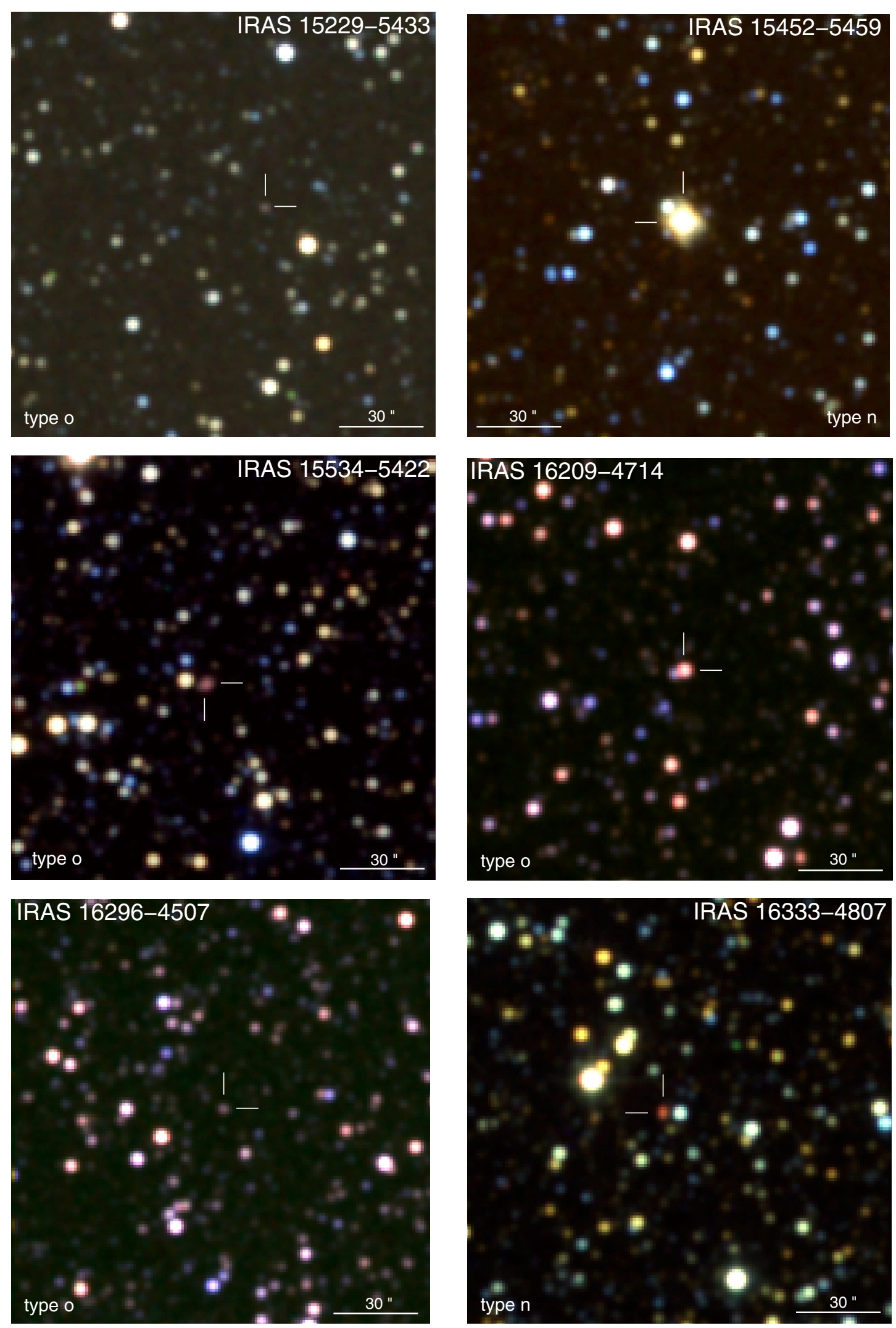

Fig. 4. continued. 

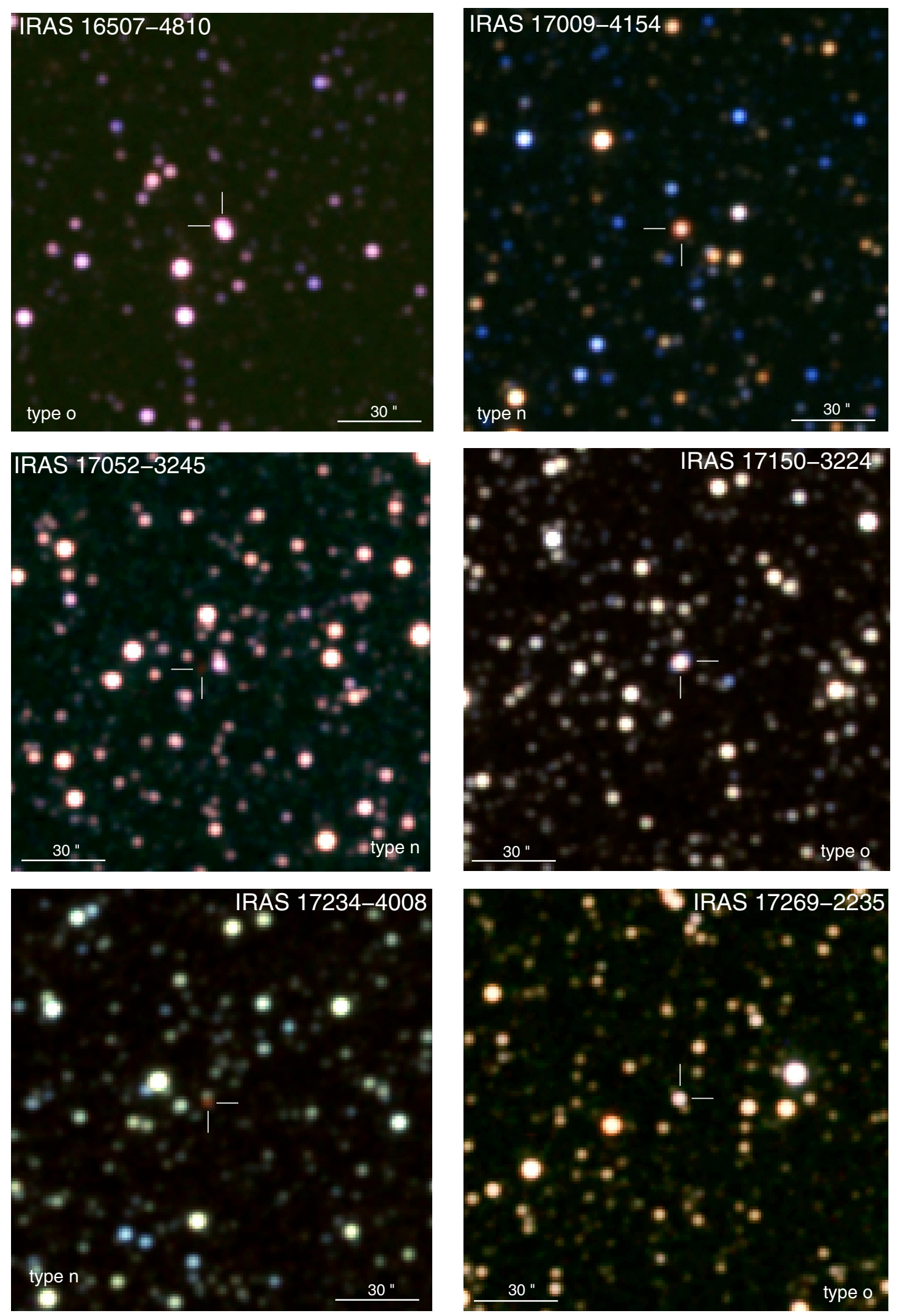

Fig. 4. continued. 
G. Ramos-Larios et al.: IRAS obscured post-AGB star and PN candidates with 2MASS counterparts. I.
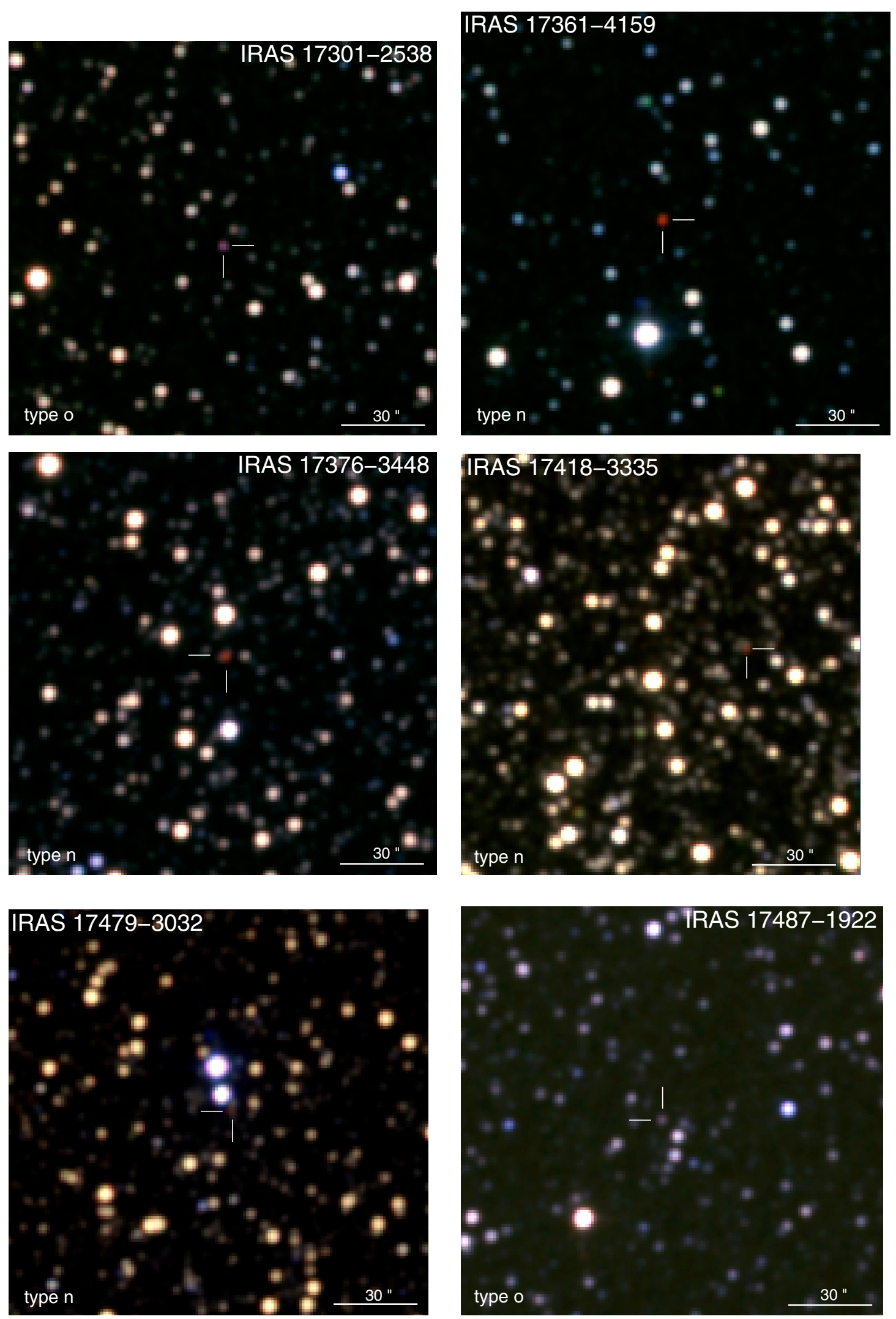

Fig. 4. continued. 

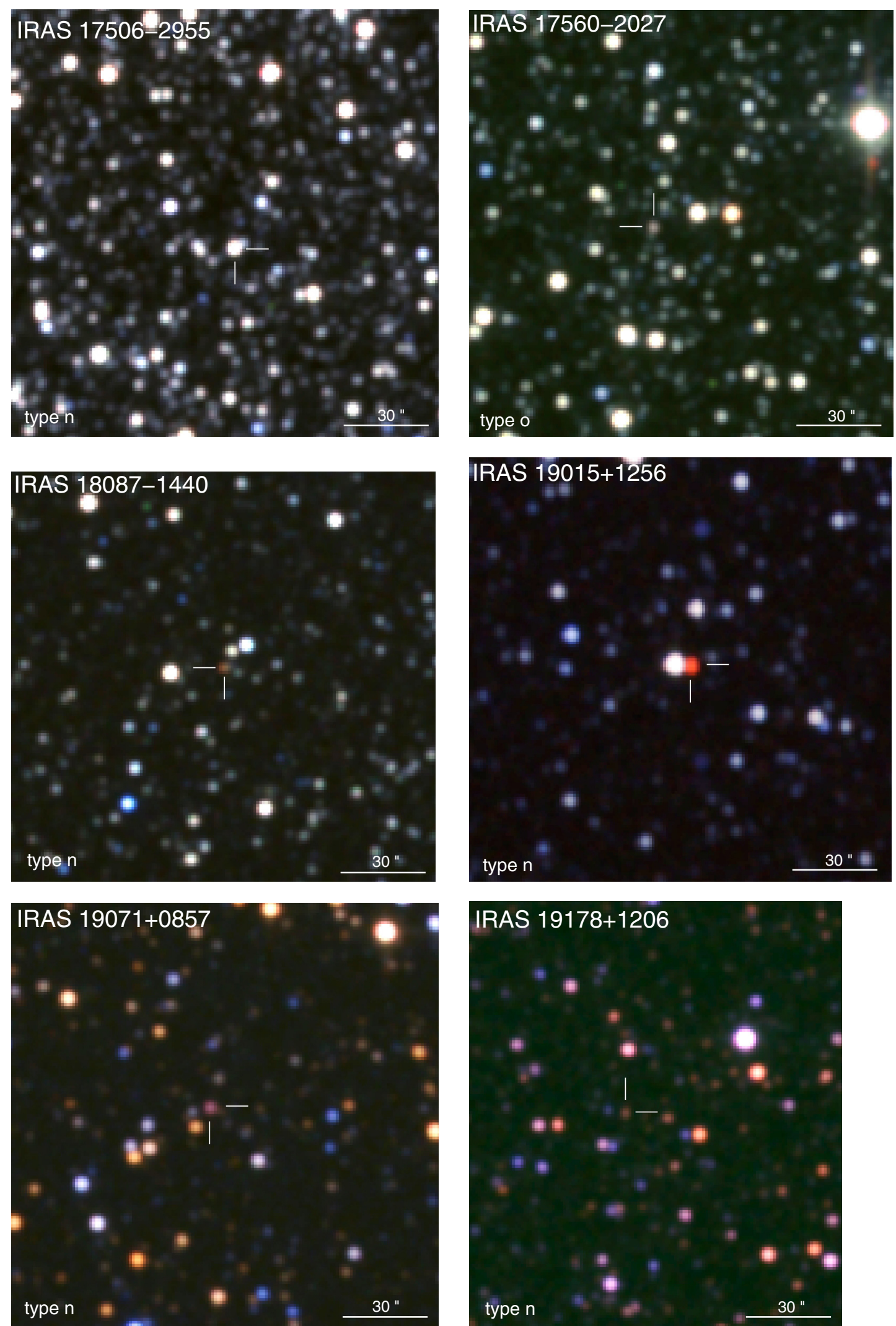

Fig. 4. continued. 
G. Ramos-Larios et al.: IRAS obscured post-AGB star and PN candidates with 2MASS counterparts. I.
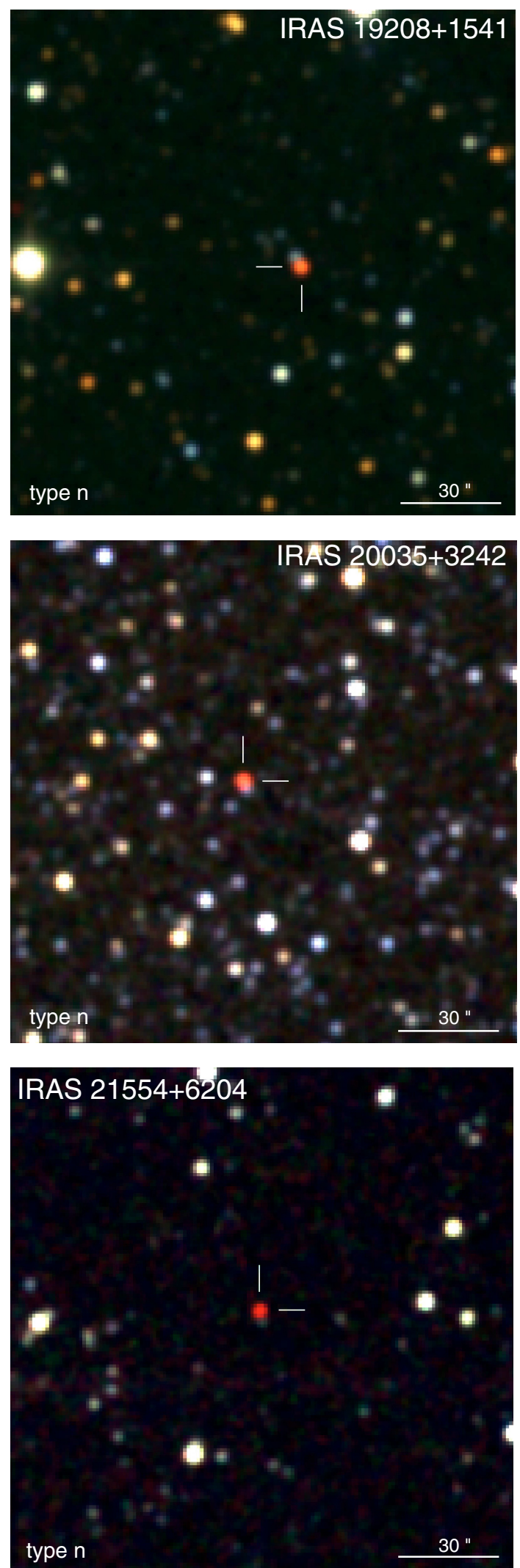

Fig. 4. continued. 

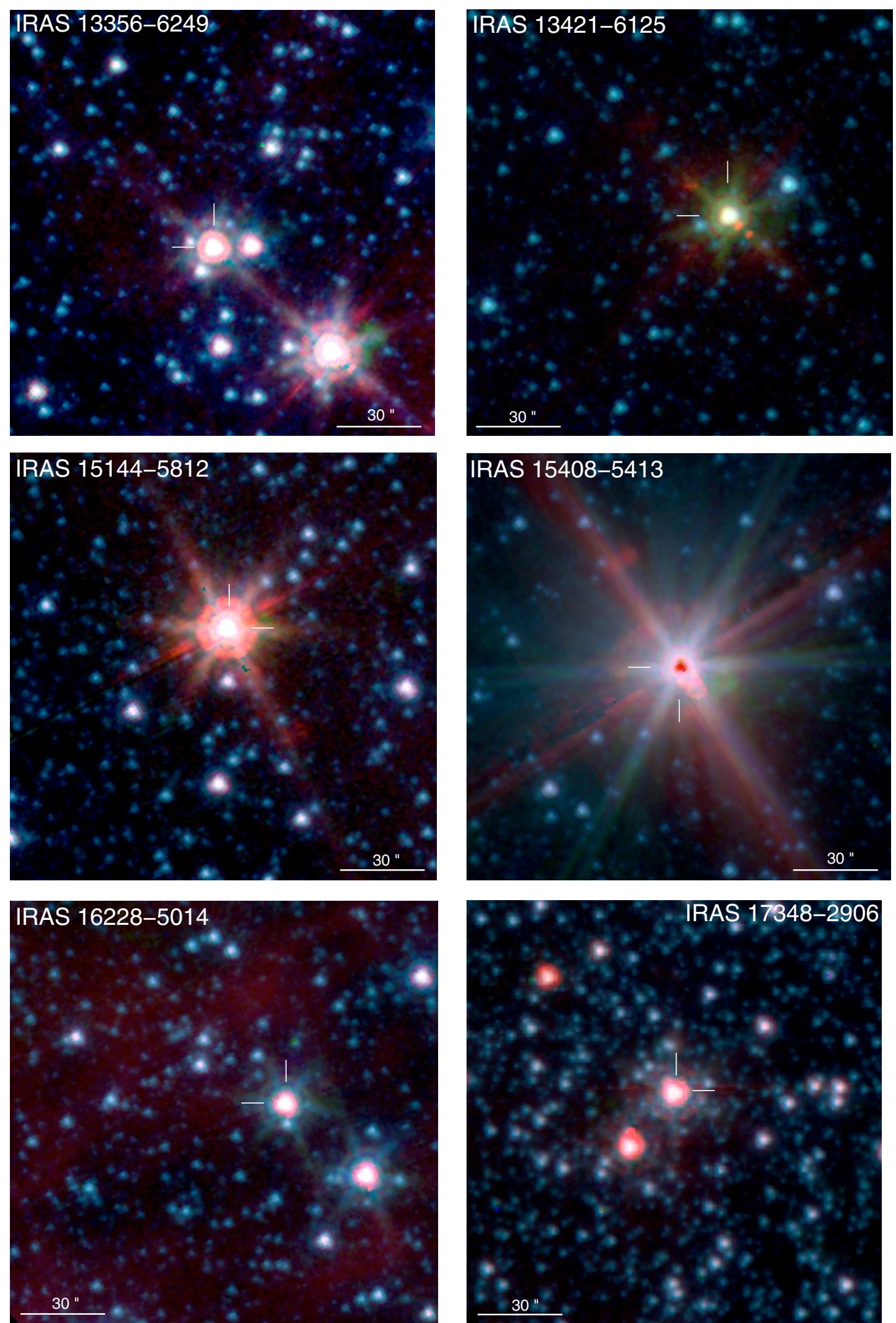

Fig. 8. Spitzer GLIMPSE composite pictures of the IRAS post-AGB star and PN candidates with near-IR counterparts in the $2 \mathrm{MASS}$ PSC and Spitzer observations. The blue, green, and red colors in the composite pictures correspond to the $3.6 \mu \mathrm{m}, 4.5 \mu \mathrm{m}$, and $8.0 \mu \mathrm{m}$ bands, respectively, except for the pictures of IRAS 13421-6125, IRAS 15408-5413, IRAS 19374+2359, and IRAS 18576+0341 where they represent the $3.6 \mu \mathrm{m}$, $4.5 \mu \mathrm{m}$, and $5.8 \mu \mathrm{m}$ bands, respectively, and for the picture of IRAS 17548-2753 where the $4.5 \mu \mathrm{m}$ band is shown in blue and green, and the $8.0 \mu \mathrm{m}$ band is shown in red. The locations of the sources are overlaid on the pictures. North is up, east to the left. 
G. Ramos-Larios et al.: IRAS obscured post-AGB star and PN candidates with 2MASS counterparts. I.
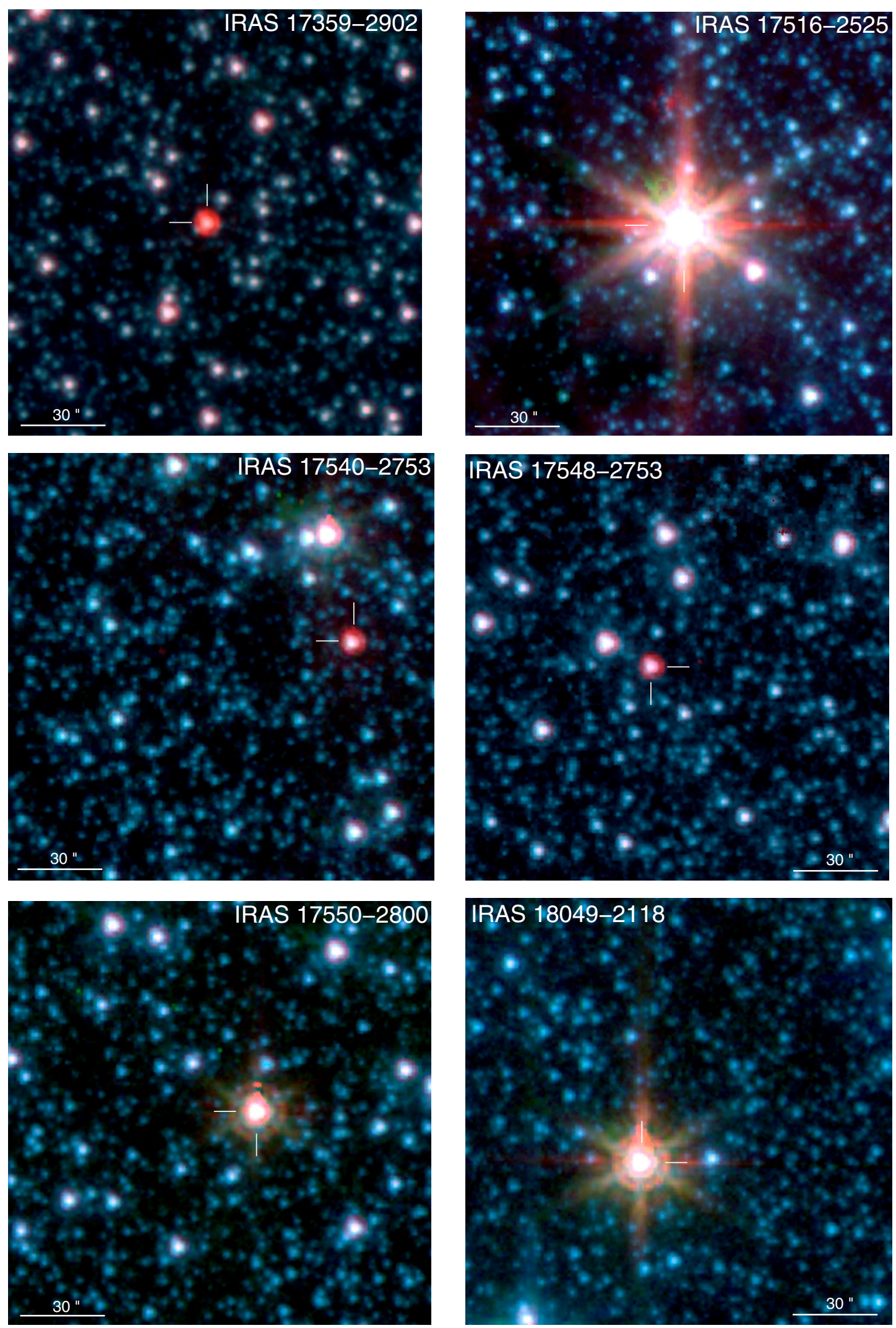

Fig. 8. continued. 

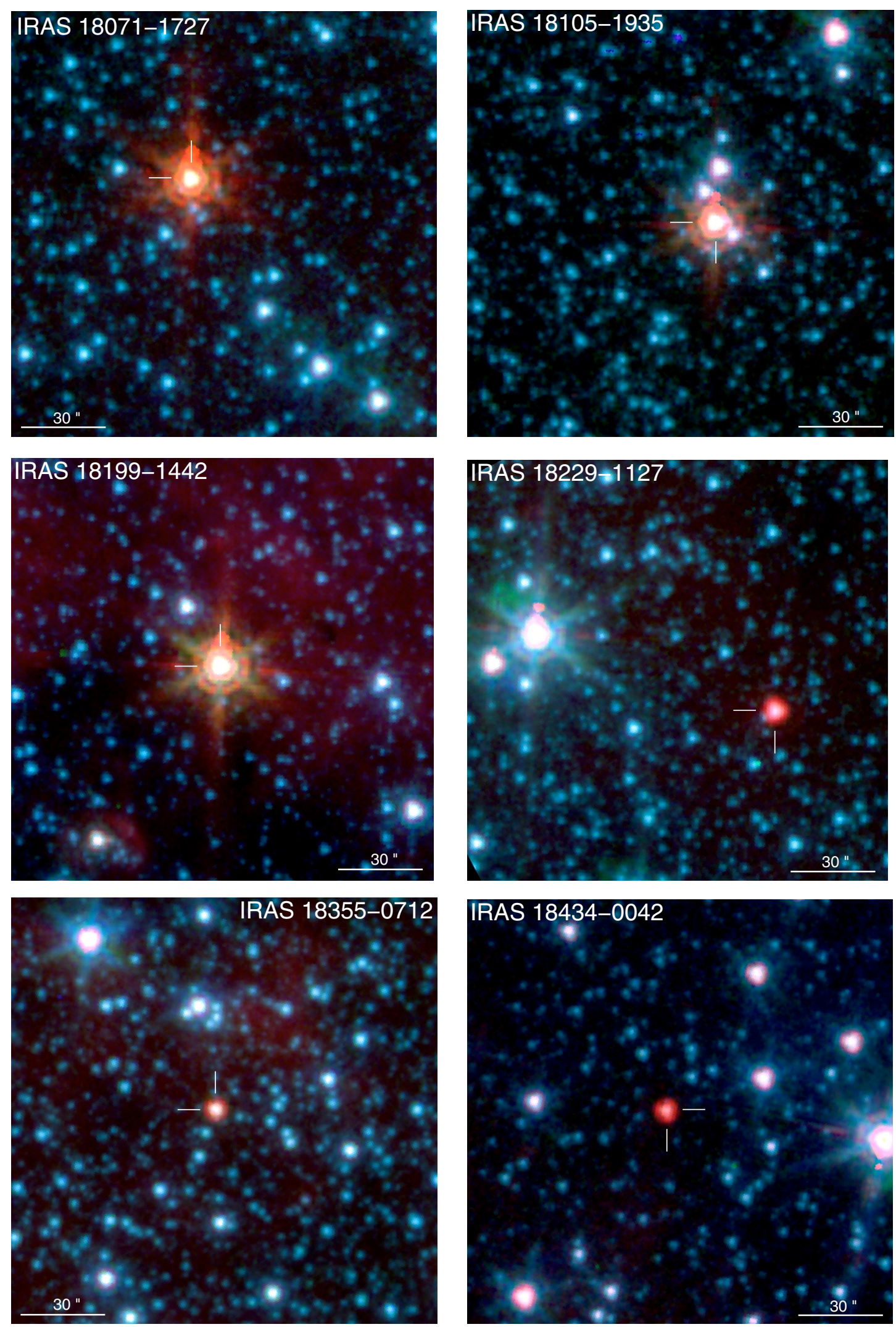

Fig. 8. continued. 
G. Ramos-Larios et al.: IRAS obscured post-AGB star and PN candidates with 2MASS counterparts. I.
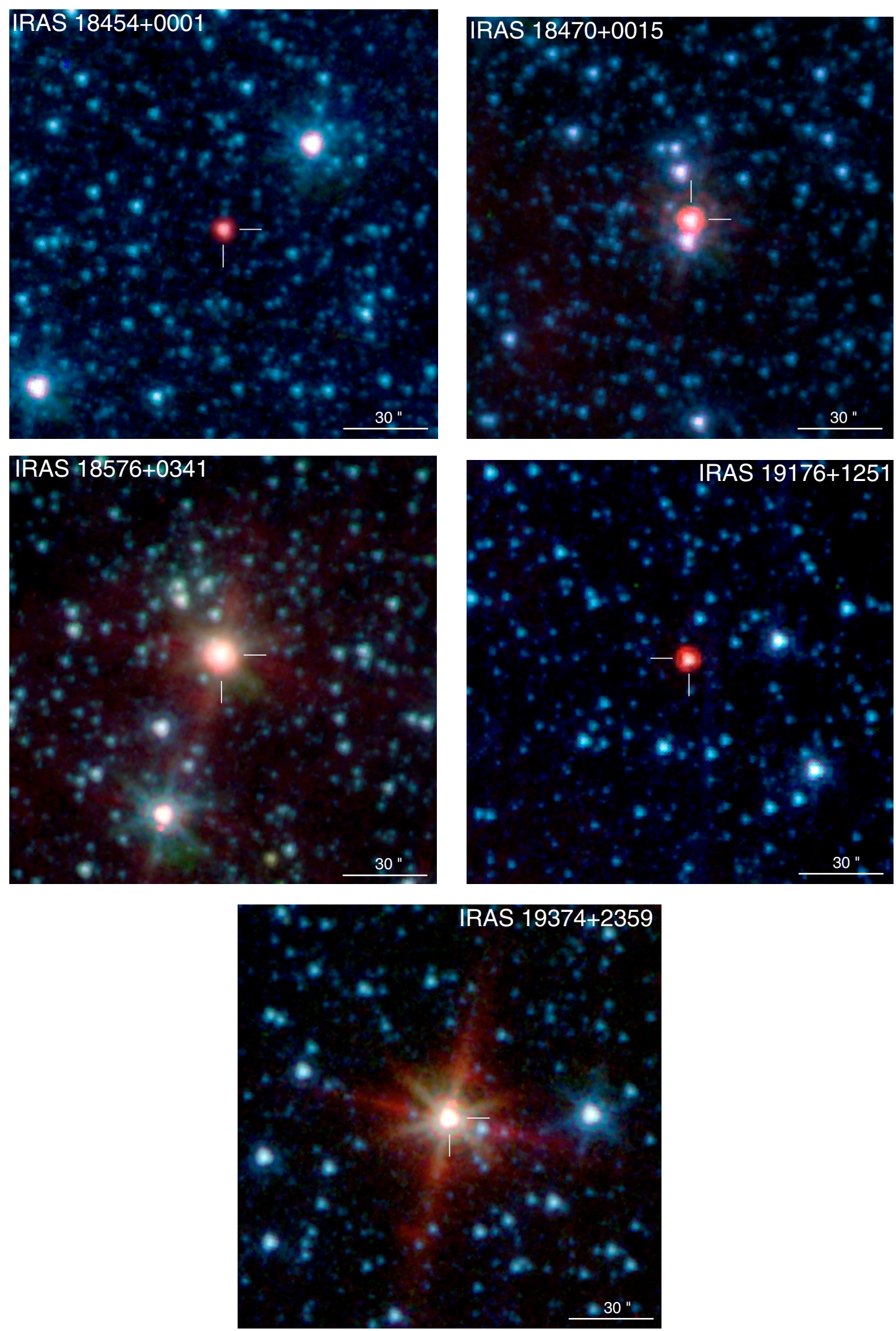

Fig. 8. continued. 